Supporting Information

\title{
Two-Step Formal [3+2] Cycloaddition of Enones/Enals and Allenyl MOM Ether: Gold-Catalyzed Highly Diastereoselective Synthesis of Cyclopentanone Enol Ether Containing an All-Carbon Quaternary Center
}

\author{
Xiaogen Huang and Liming Zhang* \\ Department of Chemistry/216, University of Nevada, Reno \\ 1664 North Virginia Street, Reno, Nevada 89557
}


General: Ethyl acetate (ACS grade), hexanes (ACS grade) and diethyl ether (ACS grade) were purchased from Fisher Scientific and used without further purification. Anhydrous tetrahydrofuran and anhydrous dichloromethane in Pure-Pac ${ }^{\mathrm{TM}}$ from Aldrich were used directly without further treatment. Commercially available reagents were used without further purification. Compounds $\mathbf{5} \mathbf{b}^{1}, \mathbf{5} \mathbf{c}^{2}, \mathbf{5} \mathbf{f}^{3}, \mathbf{5} \mathbf{h}^{3}, \mathbf{5} \mathbf{i}^{4}, \mathbf{5} \mathbf{k}^{5}, \mathbf{5 \mathbf { l } ^ { 6 }}, \mathbf{7}^{\mathbf{7}}$ and allenyl MOM ether ${ }^{8}$ were prepared according to the known procedures. Reactions were monitored by thin layer chromatography (TLC) using silicycle precoated silica gel plates. Flash column chromatography was performed over silicycle silica gel (230-400 mesh). In all cases $1 \% \mathrm{v} / \mathrm{v}$ of $\mathrm{Et}_{3} \mathrm{~N}$ was added to neutralize the silica gel to prevent the decomposition of products on column. ${ }^{1} \mathrm{H}$ NMR and ${ }^{13} \mathrm{C}$ NMR spectra were recorded on a Varian $500 \mathrm{MHz}$ Unity plus spectrometer and a Varian $400 \mathrm{MHz}$ spectrometer using residue solvent peaks as internal standards. Infrared spectra were recorded with a Perkin Elmer FT-IR spectrum 2000 spectrometer and are reported in reciprocal centimeter $\left(\mathrm{cm}^{-1}\right)$. Mass spectra were recorded with Waters micromass ZQ detector using $\mathrm{ESI}^{+}$.

Synthesis of compound $\mathbf{1}$ :<smiles>C=C=C(OC)C(O)C1=CCCCC1</smiles>

To a solution of allenyl MOM ether ( $400 \mathrm{mg}, 4 \mathrm{mmol}, 2$ eq.) was added $n$-BuLi $(1.44 \mathrm{~mL}$, $2.77 \mathrm{M}$ in hexane, $4 \mathrm{mmol}, 2$ eq.) at $-78^{\circ} \mathrm{C}$ under nitrogen. The resulting yellow solution was stirred at $-78^{\circ} \mathrm{C}$ for $1 \mathrm{~h}$. Then cyclohex-1-enecarbaldehyde (220 mg, $2 \mathrm{mmol}, 1$ eq.) in THF ( $5 \mathrm{~mL}$ ) was added dropwise. After the addition, the reaction mixture was stirred at the same temperature for $2 \mathrm{~h}$ before being quenched with water and extracted with diethyl ether $(3 \times 20 \mathrm{~mL})$. The organic layers were combined, washed with brine $(20 \mathrm{~mL})$, dried over anhydrous $\mathrm{MgSO}_{4}$, filtered, and concentrated under vacuum. The residue was purified through flash column chromatography with hexanes/ethyl acetate $(3: 1)$ on $\mathrm{Et}_{3} \mathrm{~N}$-basified silica gel. The desired product 1 was isolated in $90 \%$ yield.

${ }^{1} \mathrm{H}$ NMR $\left(500 \mathrm{MHz}, \mathrm{CDCl}_{3}\right)$ ס: 5.84-5.80 (m, 1H), $5.55(\mathrm{~s}, 1 \mathrm{H}), 5.54(\mathrm{~s}, 1 \mathrm{H}), 4.85(\mathrm{~d}, 1 \mathrm{H}, \mathrm{J}$ $=6.0 \mathrm{~Hz}), 4.84(\mathrm{~d}, 1 \mathrm{H}, \mathrm{J}=6.0 \mathrm{~Hz}), 4.53(\mathrm{~d}, 1 \mathrm{H}, \mathrm{J}=5.0 \mathrm{~Hz}), 3.41(\mathrm{~s}, 3 \mathrm{H}), 2.10-1.90(\mathrm{~m}$, $5 \mathrm{H}), 1.65-1.50(\mathrm{~m}, 4 \mathrm{H}) .{ }^{13} \mathrm{C} \mathrm{NMR}\left(125 \mathrm{MHz}, \mathrm{CDCl}_{3}\right) \delta: 197.0,136.6,130.7,124.7,94.5$, 92.2, 74.6, 56.4, 25.1, 24.9, 22.6, 22.4. IR (neat): 3451, 2928, 2857, 2837, 1960, 1594, 
1450, 1438, 1404, 1270, 1145, 1077, 1004, 924; $M S\left(E^{+}\right)$Calculated for $\left[\mathrm{C}_{12} \mathrm{H}_{18} \mathrm{O}_{3} \mathrm{Na}\right]^{+}$ 233.2; Found: 232.9.

Synthesis of compound TMS-1:<smiles>C=C=C(OC)C(O[Na])C1=CCCCC1</smiles>

Compound 1 ( $210 \mathrm{mg}, 1 \mathrm{mmol}, 1$ eq.) was dissolved in anhydrous dichloromethane (10 $\mathrm{mL}$ ). $\mathrm{Et}_{3} \mathrm{~N}$ (0.42 mL, $3 \mathrm{mmol}, 3$ eq.) and $\mathrm{TMSCl}(0.26 \mathrm{~mL}, 2 \mathrm{mmol}, 2$ eq.) were added sequentially. The resulting mixture was stirred at $\mathrm{rt}$ for $1 \mathrm{~h}$. It was then quenched with water and extracted with diethyl ether $(3 \times 10 \mathrm{~mL})$. The combined organic layers were washed with brine and dried over anhydrous $\mathrm{MgSO}_{4}$. It was concentrated and the residue was purified through flash column chromatography with hexanes/ethyl acetate (20:1) on $\mathrm{Et}_{3} \mathrm{~N}$-basified silica gel. The desired product was obtained in $92 \%$ yield.

${ }^{1} \mathrm{H}$ NMR $\left(400 \mathrm{MHz}, \mathrm{CDCl}_{3}\right)$ ס: 5.84-5.80 (m, 1H), 5.45-5.40 (m, 2H), 4.84-4.80 (m, 2H), 4.49-4.47 (m, 1H), $3.40(\mathrm{~s}, 3 \mathrm{H}), 2.10-1.90(\mathrm{~m}, 4 \mathrm{H}), 1.65-1.50(\mathrm{~m}, 4 \mathrm{H}), 0.16(\mathrm{~s}, 9 \mathrm{H}) .{ }^{13} \mathrm{C}$ NMR (125 MHz, $\left.\mathrm{CDCl}_{3}\right)$ ס: 198.4, 136.6, 130.5, 123.1, 94.2, 90.1, 75.7, 56.2, 24.9, 24.6, 22.5, 22.4. IR (neat): 2963, 2360, 2342, 1505, 1362, 1262, 1174, 1036, 980; MS (ES $\left.{ }^{+}\right)$ Calculated for $\left[\mathrm{C}_{15} \mathrm{H}_{26} \mathrm{O}_{3} \mathrm{SiNa}\right]^{+}$305.2; Found: 305.1 .

Preparation of compound $\mathbf{3}$ :<smiles>COC1=CCOC1C1=CCCCC1</smiles>

To a solution of compound 1 ( $40 \mathrm{mg}, 0.19 \mathrm{mmol}, 1$ eq.) in $5 \mathrm{~mL}$ of THF was added dichloro(pyridine-2-carboxylato)Au(III) 4 (3,7 $\mathrm{mg}, 0.0095 \mathrm{mmol}, 0.05$ eq.) at RT. The resulting mixture was stirred at $\mathrm{rt}$ for $10 \mathrm{~min}$. It was quenched with $\mathrm{Et}_{3} \mathrm{~N}$ and concentrated on vacuum. The residue was purified through flash column chromatography with hexanes/ethyl acetate (50:1) on $\mathrm{Et}_{3} \mathrm{~N}$-basified silica gel. The product 3 was obtained in $75 \%$ yield.

${ }^{1} \mathrm{H} \mathrm{NMR}\left(400 \mathrm{MHz}, \mathrm{CDCl}_{3}\right)$ ס: 5.80-5.76 (m, $\left.1 \mathrm{H}\right), 5.01-4.92(\mathrm{~m}, 3 \mathrm{H}), 4.90-4.86(\mathrm{~m}, 1 \mathrm{H})$, 4.68-4.60 (m, 2H), $3.42(\mathrm{~s}, 3 \mathrm{H}), 2.10-2.04(\mathrm{~m}, 2 \mathrm{H}), 1.89-1.82(\mathrm{~m}, 1 \mathrm{H}), 1.67-1.50(\mathrm{~m}, 3 \mathrm{H})$. ${ }^{13} \mathrm{C} \mathrm{NMR}\left(125 \mathrm{MHz}, \mathrm{CDCl}_{3}\right) \delta: 152.4,136.8,127.3,95.5,94.7,86.7,73.6,56.3,25.2$, 
22.6, 22.4. IR (neat): 3371, 2964, 2928, 2896, 2037, 2024, 1956, 1615, 1322, 1177 , 1063, 918; MS (ES ${ }^{+}$Calculated for $\left[\mathrm{C}_{12} \mathrm{H}_{18} \mathrm{O}_{3} \mathrm{SiNa}\right]^{+}$233.1; Found: 233.0 .

\section{General procedure for two-step formal [3+2] cycloaddition of enals/enones and allenyl MOM ether}

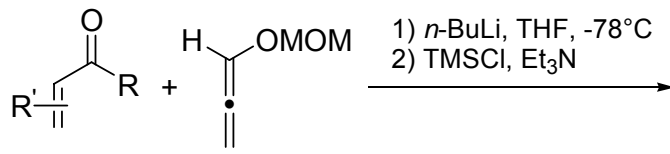

5

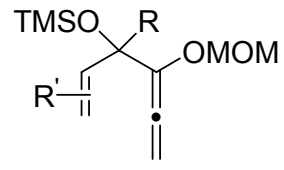

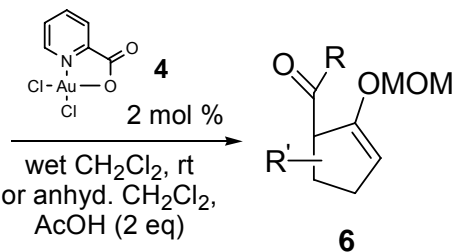

First step: synthesis of TMS-silylated allenyl carbinol:

To a solution of allenyl MOM ether (400 mg, $4 \mathrm{mmol}, 2$ eq.) was added BuLi $(1.37 \mathrm{ml}$, $2.77 \mathrm{M}$ in hexane, $3.8 \mathrm{mmol}, 1.9$ eq.) at $-78{ }^{\circ} \mathrm{C}$ under nitrogen. The resulting yellow solution was stirred at $-78^{\circ} \mathrm{C}$ for $1 \mathrm{~h}$. Then an enone or enal $(2 \mathrm{mmol}, 1 \mathrm{eq}$.) in THF (5 $\mathrm{mL}$ ) was added dropwise. After the addition, the reaction mixture was stirred at the same temperature for 2 3 $\mathrm{h}$ until TLC showed that all the enone or enal was reacted. It was then quenched with $\mathrm{TMSCl}(0.49 \mathrm{~mL}, 3.8 \mathrm{mmol}, 1.9$ eq.), and the resulting mixture was further treated with triethylamine $(0.3 \mathrm{~mL})$ to ensure complete silylation. The reaction mixture was warmed up to $0{ }^{\circ} \mathrm{C}$ and continued stirring for another $2 \mathrm{~h}$. It was then quenched with water $(10 \mathrm{~mL})$ and extracted with diethyl ether $(3 \times 10 \mathrm{~mL})$. The combined organic layers were washed with brine $(20 \mathrm{~mL})$ and dried over anhydrous $\mathrm{MgSO}_{4}$. After concentration, the residue was co-evaporated with $\mathrm{CH}_{2} \mathrm{Cl}_{2}(10 \mathrm{~mL})$ three times to remove trace amount of triethylamine which could deactivate the Au catalyst in the following step. The percentage of the corresponding silylated allenyl carbinol was calculated by the theoretical weight and the crude weight obtained, which was used for calculating the two-step overall yield.

\section{Second step: Au-catalyzed formation of aldehyde/ketone 6}

\section{General procedure A using wet $\mathrm{CH}_{2} \mathrm{Cl}_{2}$ :}

To a solution of the crude silylated allenyl carbinol obtained above ( $1 \mathrm{mmol}, 1$ eq.) in either wet $\mathrm{CH}_{2} \mathrm{Cl}_{2}{ }^{9}$ (20 mL) or was added dichloro(pyridine-2-carboxylato)gold(III) (2 or 5 $\mathrm{mol} \%$ ) at rt. The resulting mixture was stirred at rt for $10 \mathrm{~min}$. It was then quenched with a few drops of $\mathrm{Et}_{3} \mathrm{~N}$ and concentrated on vacuum. The residue was purified through flash 
column chromatography with hexanes/ethyl acetate (50:1) on $\mathrm{Et}_{3} \mathrm{~N}$-basified silica gel to give aldehyde or ketone 6 .

\section{General procedure $\mathrm{B}$ using $\mathrm{AcOH}$ in anhydrous $\mathrm{CH}_{2} \mathrm{Cl}_{2}$ :}

To a solution of the crude silylated allenyl carbinol obtained above (1 mmol, 1 eq.) and HOAc (2 mmol, 2 eq.) in anhydrous $\mathrm{CH}_{2} \mathrm{Cl}_{2}(20 \mathrm{~mL})$ was added dichloro(pyridine-2carboxylato)gold(III) ( 2 or $5 \mathrm{~mol} \%$ ) at $0^{\circ} \mathrm{C}$. The resulting mixture was stirred at the same temperature for $30 \mathrm{~min}$. It was then quenched with a few drops of $\mathrm{Et}_{3} \mathrm{~N}$ and concentrated on vacuum. The residue was purified through flash column chromatography with hexanes/ethyl acetate $(50: 1)$ on $\mathrm{Et}_{3} \mathrm{~N}$-basified silica gel to give aldehyde or ketone 6 .

Compound 2:

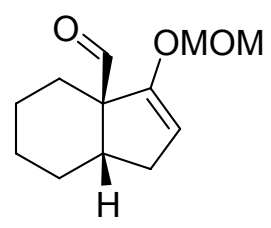

This product was prepared using the general procedure $A$ for the second step. Yield: $90 \% .{ }^{1} \mathrm{H}$ NMR $\left(400 \mathrm{MHz}, \mathrm{CDCl}_{3}\right) \delta: 9.49(\mathrm{~s}, 1 \mathrm{H}), 4.94(\mathrm{~d}, 1 \mathrm{H}, \mathrm{J}=6.0 \mathrm{~Hz}), 4.91(\mathrm{~d}, 1 \mathrm{H}, \mathrm{J}=$ $6.0 \mathrm{~Hz}$ ), $4.85(\mathrm{t}, 1 \mathrm{H}, \mathrm{J}=2.5 \mathrm{~Hz}$ ), $3.83(\mathrm{~s}, 3 \mathrm{H}$ ), 2.47 (quintet, $1 \mathrm{H}, \mathrm{J}=6.0 \mathrm{~Hz}$ ), 2.38 (ddd, $1 \mathrm{H}, \mathrm{J}=14.4,7.2,5.6 \mathrm{~Hz}$ ), 2.03 (ddd, $1 \mathrm{H}, \mathrm{J}=14.8,5.6,2.4 \mathrm{~Hz}), 1.97 \sim 1.90(\mathrm{~m}, 1 \mathrm{H})$, 1.66 1.53 (m, 2H), 1.50 1.29 (m, 5H). $\left.{ }^{13} \mathrm{C} \mathrm{NMR} \mathrm{(125} \mathrm{MHz,} \mathrm{CDCl}_{3}\right)$ ठ: 202.1, 155.9, 99.1, 94.7, 60.6, 55.9, 36.5, 32.4, 27.8, 25.3, 22.1, 21.3. IR (neat): 3419, 2932, 2855, 2707 , 2357, 1722, 1640, 1593, 1447, 1301, 1229, 1155, 1064, 979; MS (ES ${ }^{+}$) Calculated for $\left[\mathrm{C}_{12} \mathrm{H}_{18} \mathrm{O}_{3} \mathrm{Na}\right]^{+}$233.1.; Found: 233.1.

\section{Compound 6a:}

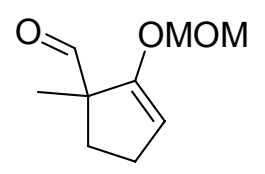

This product was prepared using the general procedure $A$ for the second step. Yield: 89\%. ${ }^{1} \mathrm{H}$ NMR $\left(400 \mathrm{MHz}, \mathrm{CDCl}_{3}\right) \delta: 9.54(\mathrm{~s}, 1 \mathrm{H}), 4.92(\mathrm{~d}, 1 \mathrm{H}, \mathrm{J}=6.0 \mathrm{~Hz}), 4.91(\mathrm{~d}, 1 \mathrm{H}, \mathrm{J}=$ $6.0 \mathrm{~Hz}$ ), $4.89(\mathrm{t}, 1 \mathrm{H}, \mathrm{J}=2.4 \mathrm{~Hz}), 3.80(\mathrm{~s}, 3 \mathrm{H}), 2.36 \sim 2.31(\mathrm{~m}, 2 \mathrm{H}), 2.29(\mathrm{q}, 1 \mathrm{H}, \mathrm{J}=6.4 \mathrm{~Hz})$, 1.70-1.63 (m, 1H), 1.25 (s, 3H). ${ }^{13} \mathrm{C}$ NMR (125 MHz, $\left.\mathrm{CDCl}_{3}\right)$ ס: 210.9, 155.7, 100.3, 94.9, 58.7, 56.1, 31.1, 26.5, 17.7. IR (neat): 2943, 2860, 2707, 1728, 1650, 1597, 1454, 1335, 
1230, 1155, 1120, 1074, 980, 925, 786; MS $\left(\mathrm{ES}^{+}\right)$Calculated for $\left[\mathrm{C}_{9} \mathrm{H}_{14} \mathrm{O}_{3} \mathrm{Na}\right]^{+}$193.1.;

Found: 193.1.

Compound 6b:

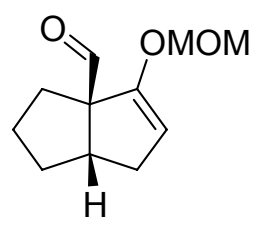

This product was prepared using the general procedure $\mathrm{A}$ for the second step. Yield: 90\%. ${ }^{1} \mathrm{H}$ NMR (400MHz, $\left.\mathrm{CDCl}_{3}\right)$ ס: $9.64(\mathrm{~s}, 1 \mathrm{H}), 4.94(\mathrm{~d}, 1 \mathrm{H}, \mathrm{J}=6.0 \mathrm{~Hz}), 4.92(\mathrm{~d}, 1 \mathrm{H}, \mathrm{J}=$ $6.0 \mathrm{~Hz}$ ), $4.82(\mathrm{t}, 1 \mathrm{H}, \mathrm{J}=2.4 \mathrm{~Hz}$ ), $3.38(\mathrm{~s}, 3 \mathrm{H}), 2.76 \sim 2.70(\mathrm{~m}, 1 \mathrm{H}), 2.64$ (ddd, $1 \mathrm{H}, \mathrm{J}=15.2$, 9.2, $2.4 \mathrm{~Hz}), 2.08 \sim 1.96(\mathrm{~m}, 2 \mathrm{H}), 1.86 \sim 1.75(\mathrm{~m}, 2 \mathrm{H}), 1.69 \sim 1.60(\mathrm{~m}, 2 \mathrm{H}), 1.46 \sim 1.39(\mathrm{~m}$, 1H). ${ }^{13} \mathrm{C}$ NMR $\left(100 \mathrm{MHz}, \mathrm{CDCl}_{3}\right) \delta: 210.9,153.4,100.4,94.9,71.4,56.1,42.8,35.4$, 35.3, 29.9, 25.9. IR (neat): 2956, 2860, 2828, 2714, 2254, 1719, 1653, 1587, 1449, 1342, $1282,1260,1208,1155,1101,1015,982 ; \mathrm{MS}\left(\mathrm{ES}^{+}\right)$Calculated for $\left[\mathrm{C}_{11} \mathrm{H}_{16} \mathrm{O}_{3} \mathrm{Na}\right]^{+}$219.1.; Found: 219.1 .

Compound 6c:

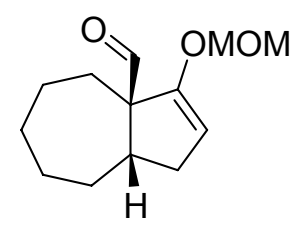

This product was prepared using the general procedure $A$ for the second step. Yield: 85\%. ${ }^{1} \mathrm{H}$ NMR $\left(400 \mathrm{MHz}, \mathrm{CDCl}_{3}\right)$ ס: $9.53(\mathrm{~s}, 1 \mathrm{H}), 4.92(\mathrm{~d}, 1 \mathrm{H}, \mathrm{J}=6.0 \mathrm{~Hz}), 4.90(\mathrm{~d}, 1 \mathrm{H}, \mathrm{J}=$ $6.0 \mathrm{~Hz}$ ), $4.85(\mathrm{t}, 1 \mathrm{H}, \mathrm{J}=2.4 \mathrm{~Hz}$ ), $3.39(\mathrm{~s}, 3 \mathrm{H}), 2.83 \sim 2.76(\mathrm{~m}, 1 \mathrm{H}), 2.57$ (ddd, $1 \mathrm{H}, \mathrm{J}=15.6$, 9.2, $2.0 \mathrm{~Hz}), 2.05 \sim 1.99(\mathrm{~m}, 2 \mathrm{H}), 1.76(\mathrm{dd}, 1 \mathrm{H}, \mathrm{J}=14.4,9.6 \mathrm{~Hz}), 1.64 \sim 1.58(\mathrm{~m}, 3 \mathrm{H})$, 1.55 1.37 (m, 5H). ${ }^{13} \mathrm{C}$ NMR $\left(100 \mathrm{MHz}, \mathrm{CDCl}_{3}\right)$ ס: 202.4, 153.1, 99.8, 94.9, 66.5, 56.2, 38.8, 34.1, 32.9, 31.4, 28.6, 27.1, 24.7. IR (neat): 3366, 2930, 2254, 1721, 1652, 1594, $1455,1225,1154,1121,906$; MS $\left(\mathrm{ES}^{+}\right)$Calculated for $\left[\mathrm{C}_{13} \mathrm{H}_{20} \mathrm{O}_{3} \mathrm{Na}\right]^{+}$247.1.; Found: 247.1. 
Compound 6d:

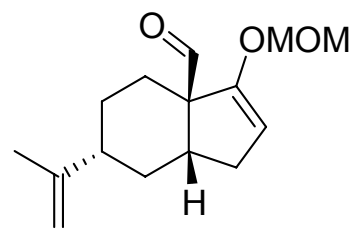

This product was prepared using the general procedure $A$ for the second step. Yield: 73\%. ${ }^{1} \mathrm{H}$ NMR $\left(400 \mathrm{MHz}, \mathrm{CDCl}_{3}\right) \delta: 9.54(\mathrm{~s}, 0.6 \mathrm{H}), 9.46(\mathrm{~s}, 0.4 \mathrm{H}), 4.98 \sim 4.64(\mathrm{~m}, 5 \mathrm{H}), 3.40$ $(\mathrm{s}, 1.2 \mathrm{H}), 3.36(\mathrm{~s}, 1.8 \mathrm{H}), 2.78 \sim 1.66(\mathrm{~m}, 13 \mathrm{H}) .{ }^{13} \mathrm{C} \mathrm{NMR}\left(125 \mathrm{MHz}, \mathrm{CDCl}_{3}\right) \delta: 202.9$, 201.2, 158.6, 152.7, 150.0, 149.7, 108.8, 108.4, 100.2, 98.2, 94.9, 94.7, 61.6, 59.6, 56.2, 56.1, 42.6, 39.9, 37.5, 37.4, 37.3, 35.9, 33.8, 31.8, 31.5, 27.7, 27.3, 26.7, 23.6, 20.9, 20.8. IR (neat): 3351, 2935, 2855, 2254, 1792, 1721, 1642, 1597, 1455, 1231, 1156, 1096, 1072, 1027, 992; MS (ES $\left.{ }^{+}\right)$Calculated for $\left[\mathrm{C}_{15} \mathrm{H}_{22} \mathrm{O}_{3} \mathrm{Na}\right]^{+}$273.1.; Found: 273.0.

Compound 6e:

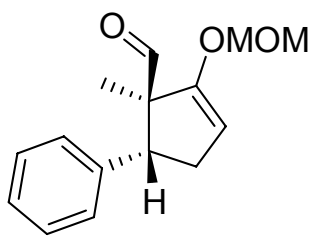

This product was prepared using the general procedure $B$ for the second step and the reaction was run at it instead of $0{ }^{\circ} \mathrm{C}$. Yield: $60 \% .{ }^{1} \mathrm{H}$ NMR $\left(400 \mathrm{MHz}, \mathrm{CDCl}_{3}\right) \delta: 9.68(\mathrm{~s}$, $1 \mathrm{H}), 7.30 \sim 7.20(\mathrm{~m}, 3 \mathrm{H}), 7.14(\mathrm{~d}, 2 \mathrm{H}, \mathrm{J}=6.8 \mathrm{~Hz}, 2 \mathrm{H}), 5.01(\mathrm{t}, 1 \mathrm{H}, \mathrm{J}=2.0 \mathrm{~Hz}), 4.98(\mathrm{~d}, 1 \mathrm{H}$, $\mathrm{J}=6.0 \mathrm{~Hz}), 4.96(\mathrm{~d}, 1 \mathrm{H}, \mathrm{J}=6.0 \mathrm{~Hz}), 3.85(\mathrm{t}, 1 \mathrm{H}, \mathrm{J}=8.4 \mathrm{~Hz}), 3.40(\mathrm{~s}, 3 \mathrm{H}), 2.74(\mathrm{dt}, 2 \mathrm{H}, \mathrm{J}$ = 8.4, $2.4 \mathrm{~Hz}), 0.86(\mathrm{~s}, 3 \mathrm{H}) \cdot{ }^{13} \mathrm{C}$ NMR $\left(125 \mathrm{MHz} \mathrm{CDCl}_{3}\right) \delta: 202.2,155.6,139.6,128.5$, 128.1, 126.7, 99.2, 94.8, 62.4, 56.1, 46.1, 32.5, 13.7. IR (neat): 3429, 3029, 2934, 2836, 2827, 2711, 1727, 1651, 1603, 1495, 1451, 1368, 1304, 1229, 1199, 1155, 1121, 1076 , 989, 967; MS (ES $\left.{ }^{+}\right)$Calculated for $\left[\mathrm{C}_{15} \mathrm{H}_{18} \mathrm{O}_{3} \mathrm{Na}\right]^{+}$269.1.; Found: 269.1.

Compound 6f:

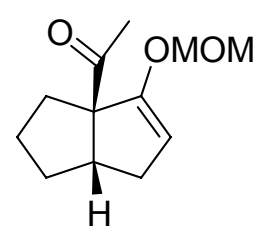


This product was prepared using the general procedure $\mathrm{B}$ for the second step. Yield: 99\%. ${ }^{1} \mathrm{H}$ NMR (400MHz, $\left.\mathrm{CDCl}_{3}\right)$ ס: $4.94(\mathrm{~d}, 1 \mathrm{H}, \mathrm{J}=6.0 \mathrm{~Hz}), 4.92(\mathrm{~d}, 1 \mathrm{H}, \mathrm{J}=6.0 \mathrm{~Hz}), 4.80$ (t, $1 \mathrm{H}, J=2.4 \mathrm{~Hz}$ ), $3.39(\mathrm{~s}, 3 \mathrm{H}), 2.72(\mathrm{ddd}, 1 \mathrm{H}, \mathrm{J}=15.6,8.8,2.0 \mathrm{~Hz}), 2.64 \sim 2.28(\mathrm{~m}, 1 \mathrm{H})$, 2.21 2.14 (m, 4H), $2.02(\mathrm{dt}, 1 \mathrm{H}, \mathrm{J}=15.6,0.8 \mathrm{~Hz}), 1.85 \sim 1.76(\mathrm{~m}, 1 \mathrm{H}), 1.70 \sim 1.54(\mathrm{~m}, 3 \mathrm{H})$, 1.42 1.36 (m, 1H). ${ }^{13} \mathrm{C}$ NMR $\left(100 \mathrm{MHz}, \mathrm{CDCl}_{3}\right)$ ס: 209.8, 155.8, 99.8, 94.9, 72.2, 56.3, 44.9, 35.9, 35.7, 31.2, 26.2, 25.9. IR (neat): 3399, 2950, 2852, 1708, 1651, 1447, 1353, 1228, 1156, 1074, 990; MS (ES $\left.{ }^{+}\right)$Calculated for $\left[\mathrm{C}_{12} \mathrm{H}_{18} \mathrm{O}_{3} \mathrm{Na}\right]^{+}$233.1.; Found: 233.1 .

Compound 6g:

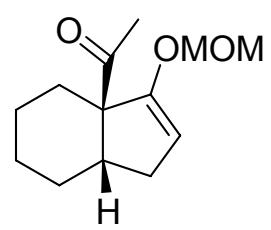

This product was prepared using the general procedure $\mathrm{B}$ for the second step. Yield: 90\%. ${ }^{1} \mathrm{H}$ NMR (400MHz, $\mathrm{CDCl}_{3}$ ) $\delta: 4.98(\mathrm{~d}, 1 \mathrm{H}, \mathrm{J}=6.0 \mathrm{~Hz}$ ), $4.95(\mathrm{~d}, 1 \mathrm{H}, \mathrm{J}=6.0 \mathrm{~Hz}), 4.78$ (t, $1 \mathrm{H}, \mathrm{J}=2.4 \mathrm{~Hz}$ ), $3.43(\mathrm{~s}, 3 \mathrm{H}$ ), 2.61 (quintet, $1 \mathrm{H}, \mathrm{J}=6.0 \mathrm{~Hz}$ ), 2.33 (ddd, $1 \mathrm{H}, \mathrm{J}=10.0$, 6.8, $2.0 \mathrm{~Hz}$ ), 2.16 (s, 3H), 2.00 (ddd, $1 \mathrm{H}, \mathrm{J}=14.4,5.6,2.4 \mathrm{~Hz}$ ), $1.87(\mathrm{~m}, 1 \mathrm{H}), 1.67 \sim 1.54$ $(\mathrm{m}, 3 \mathrm{H}), 1.52 \sim 1.32(\mathrm{~m}, 4 \mathrm{H}) .{ }^{13} \mathrm{C}$ NMR $\left(125 \mathrm{MHz}, \mathrm{CDCl}_{3}\right)$ ס: 210.2, 157.9, 98.6, 94.9, 62.1, 56.4, 38.5, 32.5, 27.9, 27.8, 25.9, 22.1, 21.8. IR (neat): 3391, 2933, 2854, 1705, 1638, $1448,1350,1222,1200,1124,1082,1059,987$; $M S\left(E^{+}\right)$Calculated for $\left[\mathrm{C}_{13} \mathrm{H}_{20} \mathrm{O}_{3} \mathrm{Na}\right]^{+}$ 247.1.; Found: 247.1.

Compound 6h:

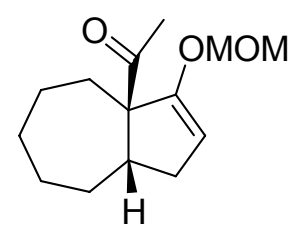

This product was prepared using the general procedure $\mathrm{B}$ for the second step. Yield: $83 \% .{ }^{1} \mathrm{H}$ NMR $\left(400 \mathrm{MHz}, \mathrm{CDCl}_{3}\right) \delta: 4.95(\mathrm{~d}, 1 \mathrm{H}, \mathrm{J}=6.0 \mathrm{~Hz}), 4.93(\mathrm{~d}, 1 \mathrm{H}, \mathrm{J}=6.0 \mathrm{~Hz}), 4.88$ (t, $1 \mathrm{H}, J=2.4 \mathrm{~Hz}$ ), $3.40(\mathrm{~s}, 3 \mathrm{H}), 2.69 \sim 2.64(\mathrm{~m}, 1 \mathrm{H}), 2.55$ (ddd, $1 \mathrm{H}, \mathrm{J}=12.0,9.6,2.4 \mathrm{~Hz}$ ), 2.17 (s, 3H), 2.10 (ddd, $1 \mathrm{H}, J=16.0,4.0,2.8 \mathrm{~Hz}$ ), 2.00 (dd, $1 \mathrm{H}, J=14.8,10.0 \mathrm{~Hz}$ ), 1.75 (dd, $1 \mathrm{H}, \mathrm{J}=14.8,8.8 \mathrm{~Hz}), 1.63 \sim 1.43(\mathrm{~m}, 6 \mathrm{H}), 1.37 \sim 1.27(\mathrm{~m}, 2 \mathrm{H}) .{ }^{13} \mathrm{C} \mathrm{NMR}(100 \mathrm{MHz}$, $\mathrm{CDCl}_{3}$ ) $\delta: 210.6,155.5,99.7,94.9,67.8,56.4,40.8,33.3,32.2,31.2,29.9,25.9,25.8$, 
24.7. IR (neat): $3389,2926,2855,2253,1702,1656,1597,1454,1353,1193,1155$, 1080, 1046, 997; MS $\left(\mathrm{ES}^{+}\right)$Calculated for $\left[\mathrm{C}_{14} \mathrm{H}_{22} \mathrm{O}_{3} \mathrm{Na}\right]^{+}$261.1.; Found: 261.1 .

Compound 6i:

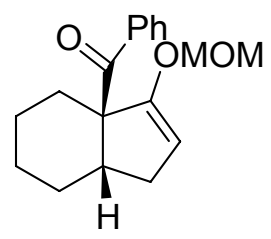

This product was prepared using the general procedure $\mathrm{B}$ for the second step. Yield: 44\%. ${ }^{1} \mathrm{H}$ NMR $\left(400 \mathrm{MHz}, \mathrm{CDCl}_{3}\right)$ ס: 7.99 7.96 (m, 2H), 7.47 7.43 (m, 1H), 7.39 7.35 (m, $2 \mathrm{H}), 4.91 \sim 4.90(\mathrm{~m}, 2 \mathrm{H}), 4.85(\mathrm{t}, 1 \mathrm{H}, \mathrm{J}=2.0 \mathrm{~Hz}), 3.27(\mathrm{~s}, 3 \mathrm{H}), 2.97 \sim 2.90(\mathrm{~m}, 1 \mathrm{H})$, 2.59 2.53 (m, 1H), 2.43 2.28 (m, 2H), 1.54 1.36 (m, 7H). ${ }^{13} \mathrm{C} \mathrm{NMR}\left(125 \mathrm{MHz}, \mathrm{CDCl}_{3}\right) \delta$ : 203.1, 161.1, 137.8, 131.4, 128.88., 127.9, 97.4, 94.9, 60.8, 56.4, 40.9, 31.6, 30.6, 26.3, 21.7, 21.3. IR (neat): 3367, 2935, 2850, 2254, 1672, 1639, 1597, 1446, 1301, 1230, 1156, 1089, 1053, 989; $\mathrm{MS}\left(\mathrm{ES}^{+}\right)$Calculated for $\left[\mathrm{C}_{18} \mathrm{H}_{22} \mathrm{O}_{3} \mathrm{Na}\right]^{+}$309.1.; Found: 309.1 .

\section{Compound 6j:}

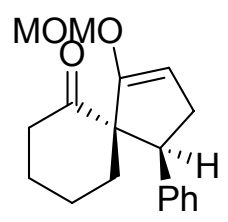

This product was prepared using the general procedure $\mathrm{B}$ for the second step. Yield: 90\%. ${ }^{1} \mathrm{H} \mathrm{NMR}\left(400 \mathrm{MHz}, \mathrm{CDCl}_{3}\right)$ ס: 7.40 7.30 (m, 5H), $5.10(\mathrm{~d}, 1 \mathrm{H}, \mathrm{J}=6.0 \mathrm{~Hz}), 5.05(\mathrm{~d}$, $1 \mathrm{H}, \mathrm{J}=6.0 \mathrm{~Hz}), 4.99(\mathrm{t}, 1 \mathrm{H}, \mathrm{J}=2.5 \mathrm{~Hz}), 4.13(\mathrm{t}, 1 \mathrm{H}, \mathrm{J}=7.5 \mathrm{~Hz}), 3.54(\mathrm{~s}, 3 \mathrm{H}), 2.74 \sim 2.70$ (m, 2H), 2.62 (ddd, $1 \mathrm{H}, \mathrm{J}=14.4,6.8,2.4 \mathrm{~Hz}$ ), $2.54(\mathrm{dt}, 1 \mathrm{H}, \mathrm{J}=14.8,6.0 \mathrm{~Hz}$ ), 1.92 1.83 $(\mathrm{m}, 3 \mathrm{H}), 1.73 \sim 1.64(\mathrm{~m}, 1 \mathrm{H}), 1.55 \sim 1.47(\mathrm{~m}, 1 \mathrm{H}), 1.38 \sim 1.30(\mathrm{~m}, 1 \mathrm{H}) .{ }^{13} \mathrm{C} \mathrm{NMR}(125 \mathrm{MHz}$, $\mathrm{CDCl}_{3}$ ) ठ: 211.7, 157.6, 141.6, 129.1, 128.1, 126.6, 98.1, 95.0, 64.1, 56.3, 48.3, 41.2, 32.7, 32.5, 25.7, 21.3. IR (neat): 3367, 2943, 2253, 2699, 1644, 1601, 1463, 1192, 1155, 1089, 1037, 990; $\mathrm{MS}\left(\mathrm{ES}^{+}\right)$Calculated for $\left[\mathrm{C}_{18} \mathrm{H}_{22} \mathrm{O}_{3} \mathrm{Na}\right]^{+}$309.1.; Found: 309.1. 
Compound 6k:

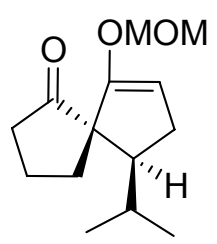

This product was prepared using the general procedure $A$ for the second step. Yield: $79 \% .{ }^{1} \mathrm{H}$ NMR $\left(400 \mathrm{MHz}, \mathrm{CDCl}_{3}\right) \delta: 4.85(\mathrm{~d}, 1 \mathrm{H}, \mathrm{J}=6.0 \mathrm{~Hz}), 4.78(\mathrm{~d}, 1 \mathrm{H}, \mathrm{J}=6.0 \mathrm{~Hz}) 4.65$ $(\mathrm{t}, 1 \mathrm{H}, \mathrm{J}=2.0 \mathrm{~Hz}), 3.33(\mathrm{~s}, 3 \mathrm{H}), 2.44 \sim 1.86(\mathrm{~m}, 10 \mathrm{H}), 1.65 \sim 1.55(\mathrm{~m}, 1 \mathrm{H}), 0.87(\mathrm{~d}, 3 \mathrm{H}, \mathrm{J}=$ $2.4 \mathrm{~Hz}), 0.69(\mathrm{~d}, 3 \mathrm{H}, \mathrm{J}=2.4 \mathrm{~Hz}) .{ }^{1} \mathrm{H}$ NMR(400MHz, benzene- $\left.d_{6}\right) \delta: 4.66(\mathrm{t}, 1 \mathrm{H}, \mathrm{J}=2.4$ $\mathrm{Hz}), 4.59(\mathrm{~d}, 1 \mathrm{H}, \mathrm{J}=6.0 \mathrm{~Hz}), 4.51(\mathrm{~d}, 1 \mathrm{H}, \mathrm{J}=6.0 \mathrm{~Hz}), 3.05(\mathrm{~s}, 3 \mathrm{H}), 2.58(\mathrm{q}, 1 \mathrm{H}, \mathrm{J}=10.0$ $\mathrm{Hz}), 2.35 \sim 2.24(\mathrm{~m}, 2 \mathrm{H}), 2.09 \sim 1.78(\mathrm{~m}, 6 \mathrm{H}), 1.65 \sim 1.59(\mathrm{~m}, 1 \mathrm{H}), 1.44 \sim 1.36(\mathrm{~m}, 1 \mathrm{H}), 0.78$

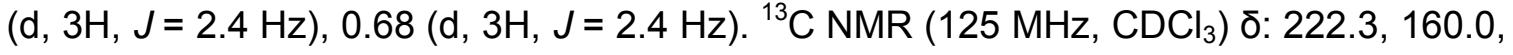
96.8, 94.5, 62.1, 56.1, 51.3, 38.1, 32.0, 29.5, 28.8, 22.3, 20.5, 19.6. IR (neat): 3366, 2962, 2253, 1730, 1651, 1597, 1468, 1226, 1151, 1079, 1000, 973; MS (ES ${ }^{+}$) Calculated for $\left[\mathrm{C}_{14} \mathrm{H}_{22} \mathrm{O}_{3} \mathrm{Na}\right]^{+}$261.1.; Found: 261.0.

Compound 6I:

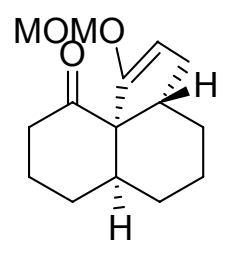

This product was prepared using the general procedure $B$ for the second step and the reaction was run at $\mathrm{rt}$ instead of $0{ }^{\circ} \mathrm{C}$. Yield: $83 \% .{ }^{1} \mathrm{H}$ NMR $\left(400 \mathrm{MHz}, \mathrm{CDCl}_{3}\right)$ ס: $4.94(\mathrm{~d}$, $1 \mathrm{H}, \mathrm{J}=6.0 \mathrm{~Hz}), 4.88(\mathrm{~d}, 1 \mathrm{H}, \mathrm{J}=6.0 \mathrm{~Hz}), 4.67(\mathrm{t}, 1 \mathrm{H}, \mathrm{J}=2.4 \mathrm{~Hz}), 3.40(\mathrm{~s}, 3 \mathrm{H}), 2.95 \sim 2.85$ $(\mathrm{m}, 1 \mathrm{H}), 2.80 \sim 2.70(\mathrm{~m}, 1 \mathrm{H}), 2.42(\mathrm{ddt}, 1 \mathrm{H}, \mathrm{J}=14.4,3.6,1.6 \mathrm{~Hz}), 2.32 \sim 2.20(\mathrm{~m}, 1 \mathrm{H})$, 2.10 2.00 (m, 3H), 1.92 1.82 (m, 2H), 1.62 1.08 (m, 7H). $\left.{ }^{13} \mathrm{C} \mathrm{NMR} \mathrm{(125} \mathrm{MHz,} \mathrm{CDCl} \mathrm{N}_{3}\right)$ : 212.0, 160.1, 96.8, 94.9, 62.4, 56.4, 42.6, 41.4, 37.1, 29.6, 28.1, 28.0, 25.9, 22.2, 21.5. IR (neat): 3398, 2930, 2254, 1699, 1597, 1455, 1118, 907; MS (ES ${ }^{+}$) Calculated for $\left[\mathrm{C}_{15} \mathrm{H}_{22} \mathrm{O}_{3} \mathrm{Na}\right]^{+}$273.1.; Found: 273.1. 
Compound 8:

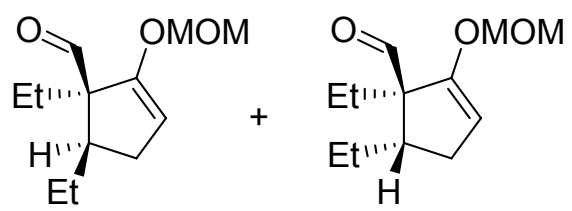

Yield: $85 \% .{ }^{1} \mathrm{H} \mathrm{NMR}\left(400 \mathrm{MHz}, \mathrm{CDCl}_{3}\right)$ ס:9.63 (s, 0.73H), 9.49 (s, 0.27H), 5.07 (t, $0.73 \mathrm{H}, \mathrm{J}$ $=2.0 \mathrm{~Hz}), 4.94(\mathrm{t}, 0.27 \mathrm{H}, \mathrm{J}=2.0 \mathrm{~Hz}), 4.92 \sim 4.88(\mathrm{~m}, 2 \mathrm{H}), 3.39(\mathrm{~s}, 2.2 \mathrm{H}), 3.37(\mathrm{~s}, 0.8 \mathrm{H})$, $2.44(\mathrm{ddd}, 0.73 \mathrm{H}, \mathrm{J}=14.5,8.5,2.0 \mathrm{~Hz}), 2.35(\mathrm{ddd}, 0.27 \mathrm{H}, \mathrm{J}=14.5,8.0,3.0 \mathrm{~Hz}$ ), 2.37 2.34 (m, 0.27H), 2.25 2.18 (m, 0.73H), $1.98(\mathrm{ddd}, 0.73 \mathrm{H}, \mathrm{J}=14.5,9.0,2.0 \mathrm{~Hz}$ ), 1.85 (ddd, $0.23 \mathrm{H}, J=14.5,9.0,2.0 \mathrm{~Hz}), 1.84 \sim 1.76(\mathrm{~m}, 1 \mathrm{H}), 1.63 \sim 1.27(\mathrm{~m}, 3 \mathrm{H})$, 0.97 0.86 (m, 6H). ${ }^{13} \mathrm{C}$ NMR $\left(125 \mathrm{MHz}, \mathrm{CDCl}_{3}\right)$ ס: 3418, 965, 2936, 2878, 2849, 2716, 2353, 2254, 1720, 1648, 1597, 1463, 1381, 1340, 1229, 1156, 1076, 985. IR (neat): 203.7, 203.0, 155.4, 154.1, 101.9, 100.7, 94.79, 94.80, 65.4, 64.4, 56.3, 56.2, 45.0, 43.1, 34.1, 33.5, 23.6, 22.2, 21.1, 20.0, 12.9, 12.6, 9.3, 8.1; MS $\left(\mathrm{ES}^{+}\right)$Calculated for $\left[\mathrm{C}_{12} \mathrm{H}_{20} \mathrm{O}_{3} \mathrm{Na}\right]^{+}$235.0; Found: 234.9 .

References:

1. Wang, Z.; Qian, X.; Zhou, W. Tetrahedron 1990, 46, 1191-1198.

2. Naya, A.; Ishikawa, M.; Matsuda, K.; Ohwaki, K.; Saeki, T.; Noguchi, K.; Ohtake, N. Bioorg. Med. Chem. 2003, 11, 875-884.

3. House, H. O.; Sayer, T. S. B.; Yau, C.-C. J. Org. Chem. 1978, 43, 2153-2157.

4. Armstrong, C.; Blair, J. A.; Homer, J. J. Chem. Soc. Chem. Commun. 1969, 3, 103-104.

5. Ijima, A.; Takahashi, K. Chem. Pharm. Bull. 1973, 21, 215.

6. Varseev, G. N.; Maier, M. E. Org. Lett. 2005, 7, 3881-3884.

7. Zacuto, M. J.; Cai, D. Tetrahedron Lett. 2005, 46, 8289-8292.

8. Chengebroyen, J.; Linke, M.; Robitzer, M.; Sirlin, C.; Pfeffer, M. J. Organomet.Chem. 2003, 687, 313-321.

9. Generated by shaking $\mathrm{CH}_{2} \mathrm{Cl}_{2}$ with $\mathrm{H}_{2} \mathrm{O}$ followed by phase separation. Also see: Zhang, L.; Wang, S. J. Am. Chem. Soc. 2006, 128, 1442-1443. 


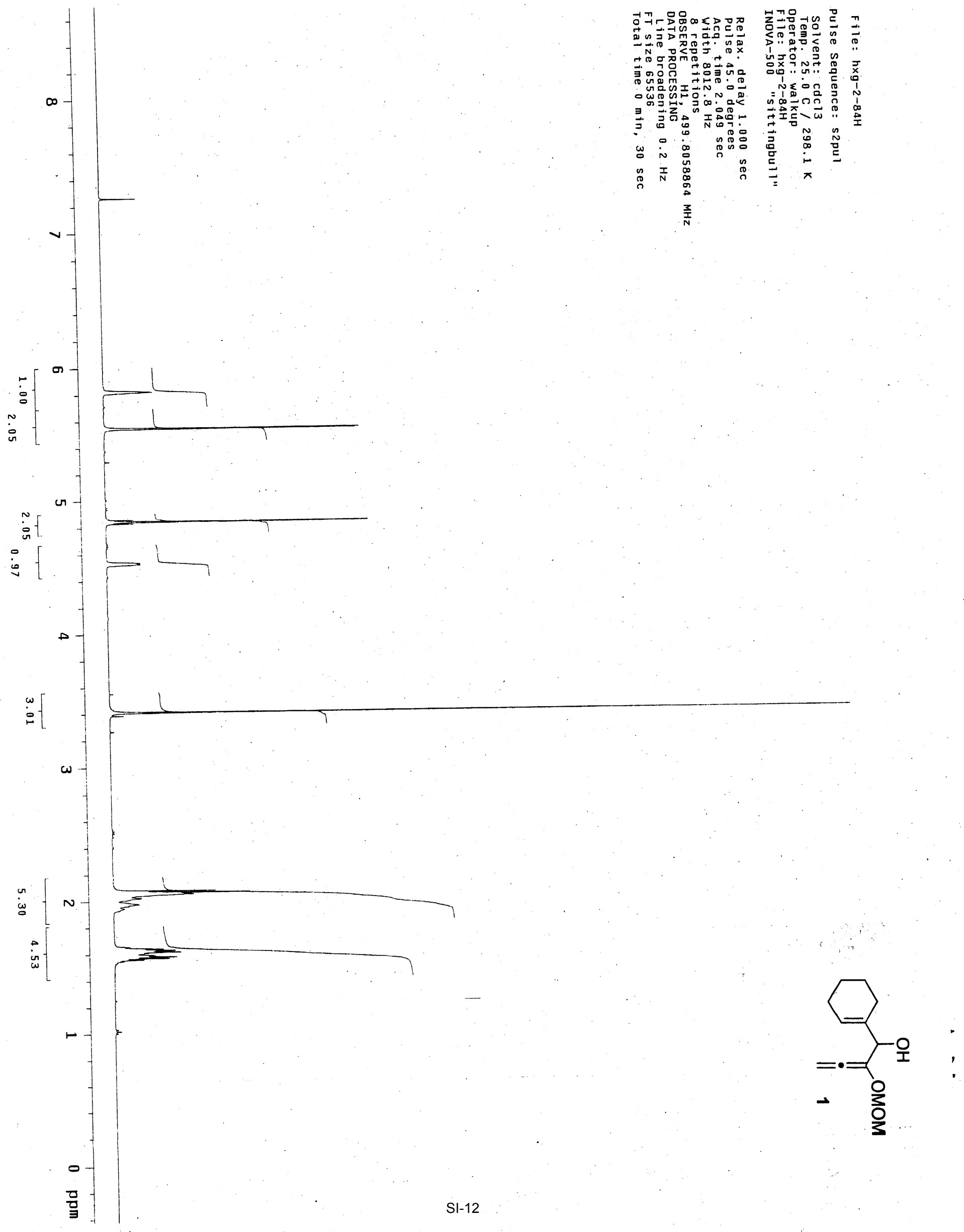



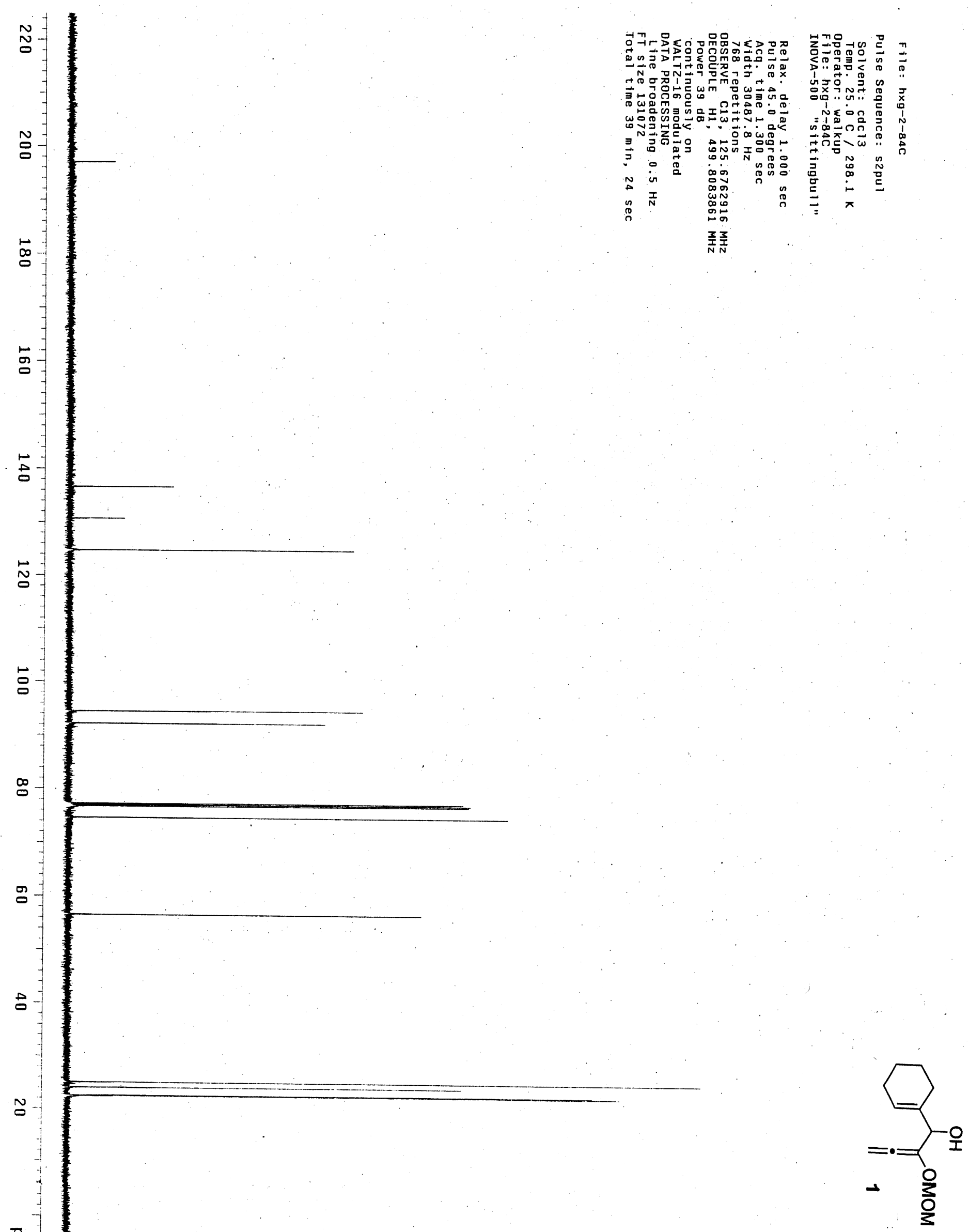


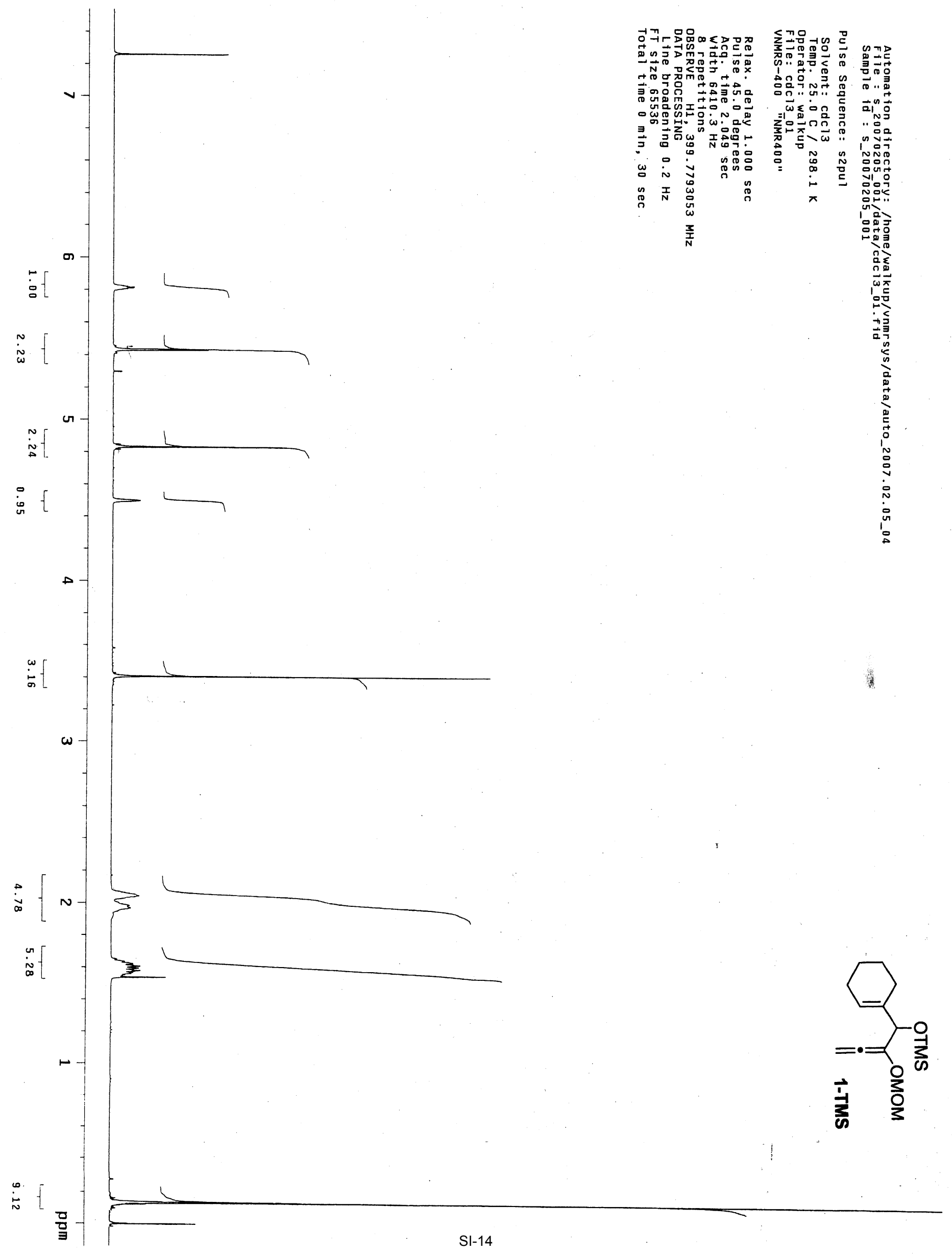



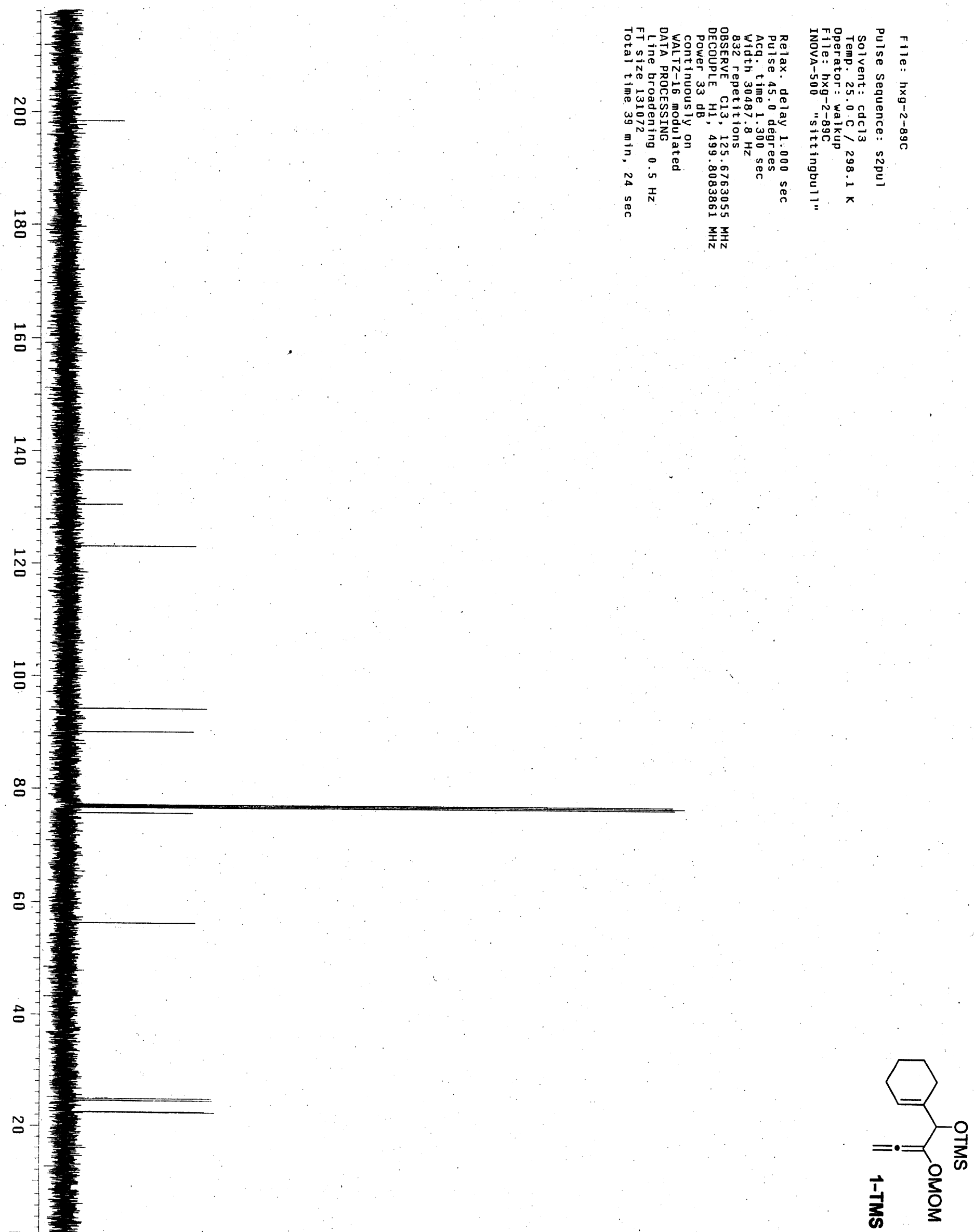


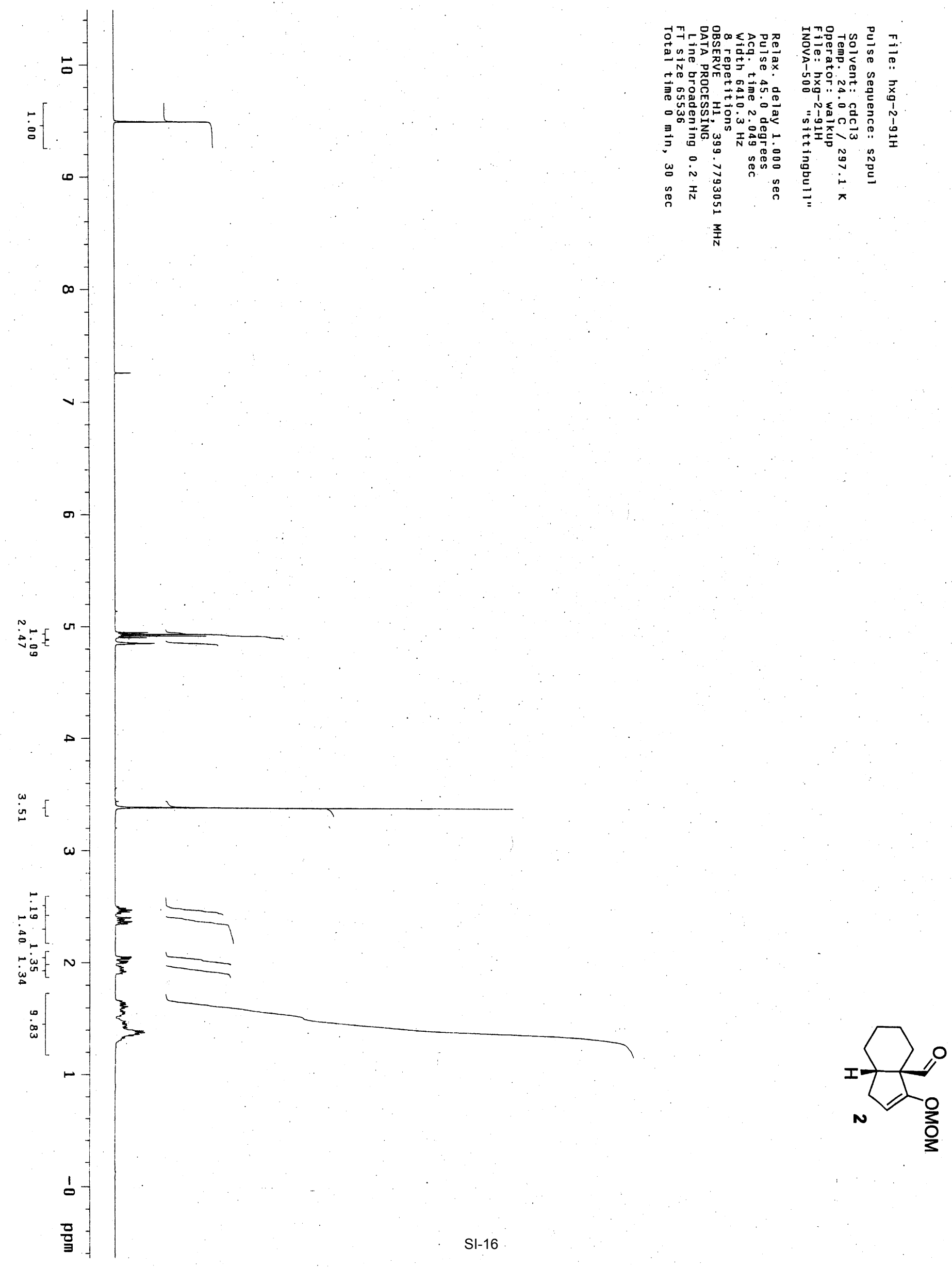




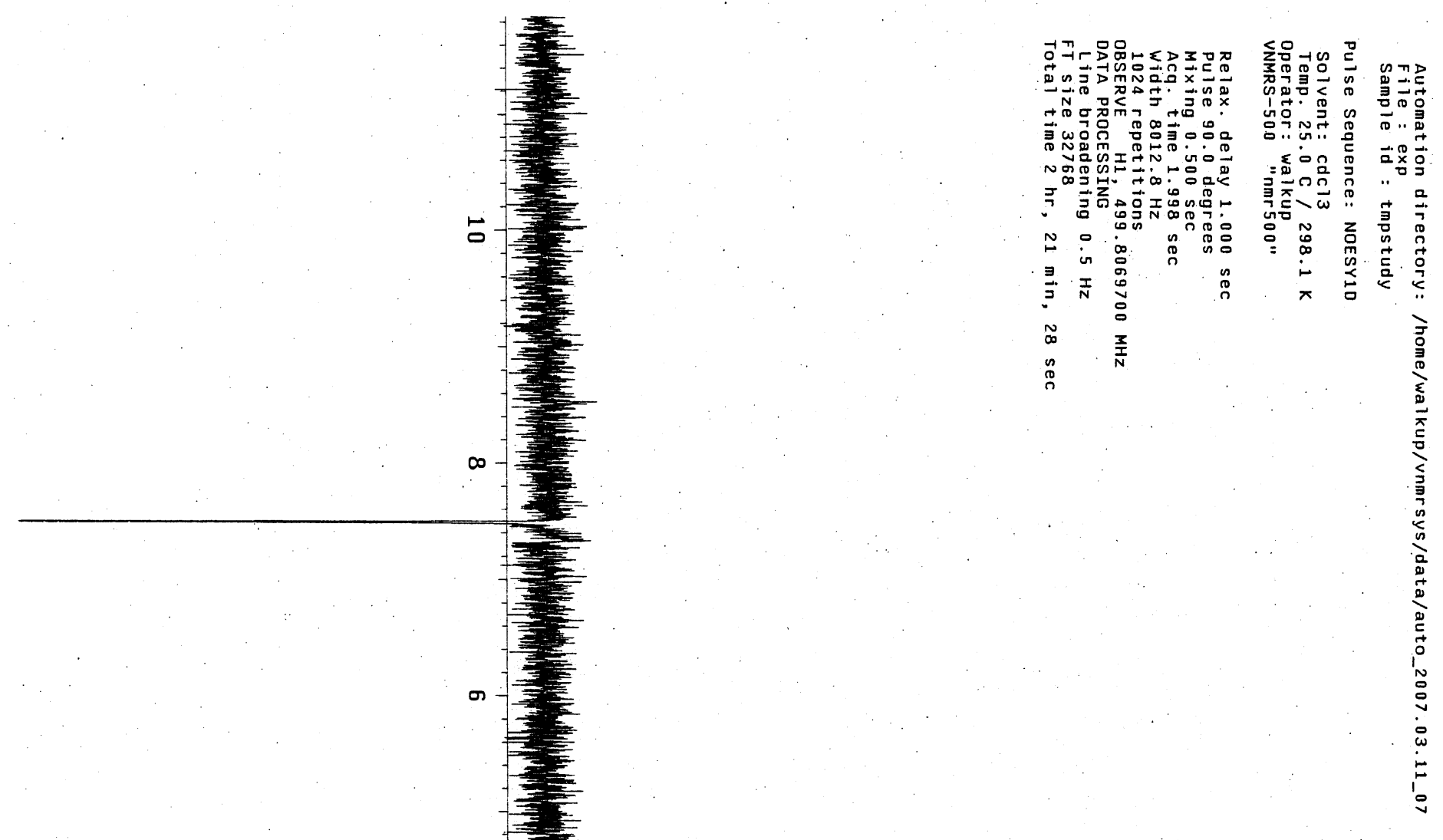




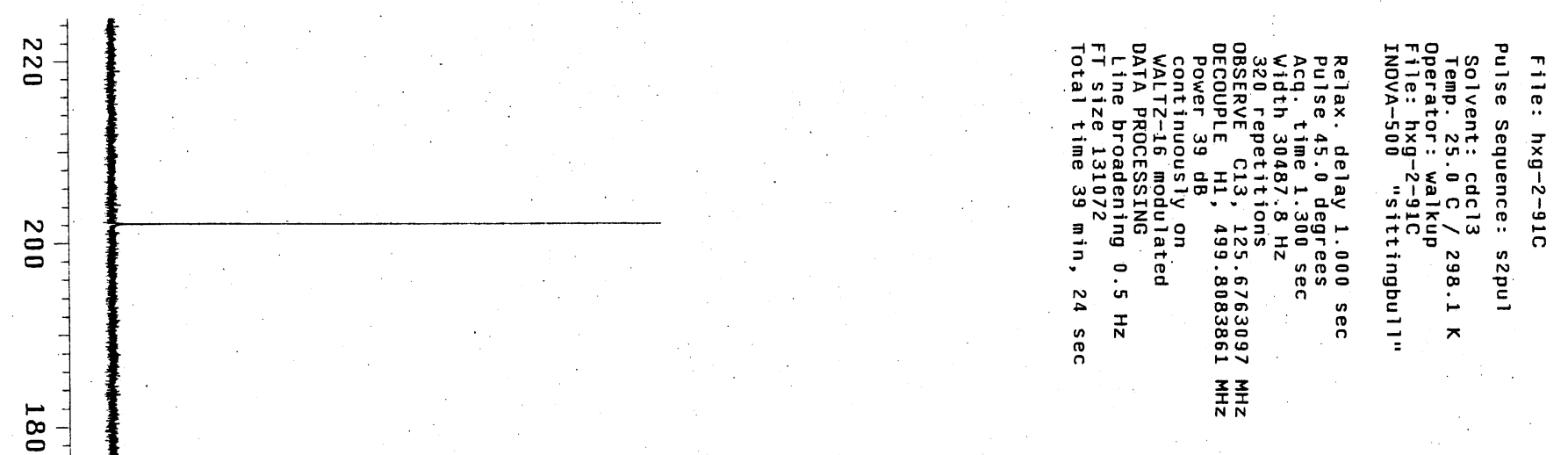

뭉

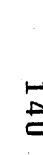

엄

옹

$\stackrel{\infty}{\circ}$

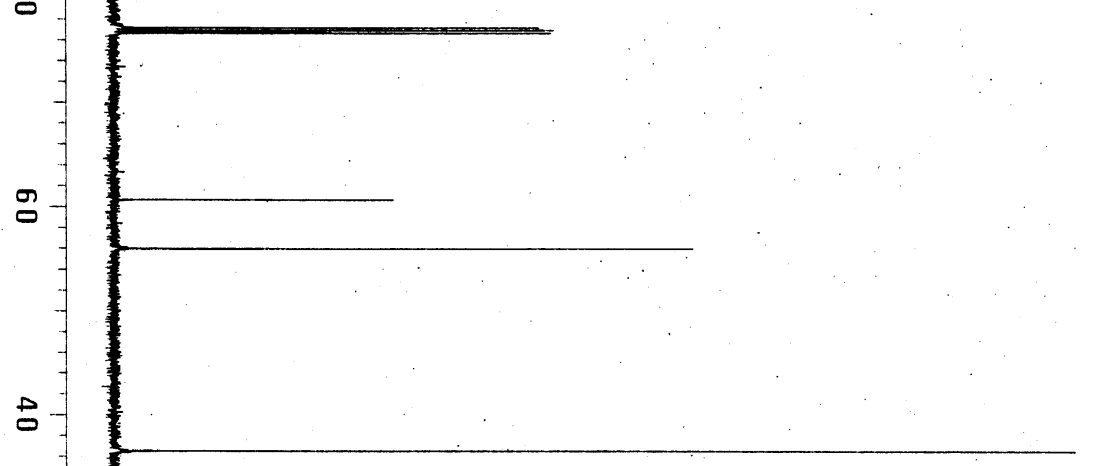

a

1
0
$j$
$j$
$j$
$j$
$j$
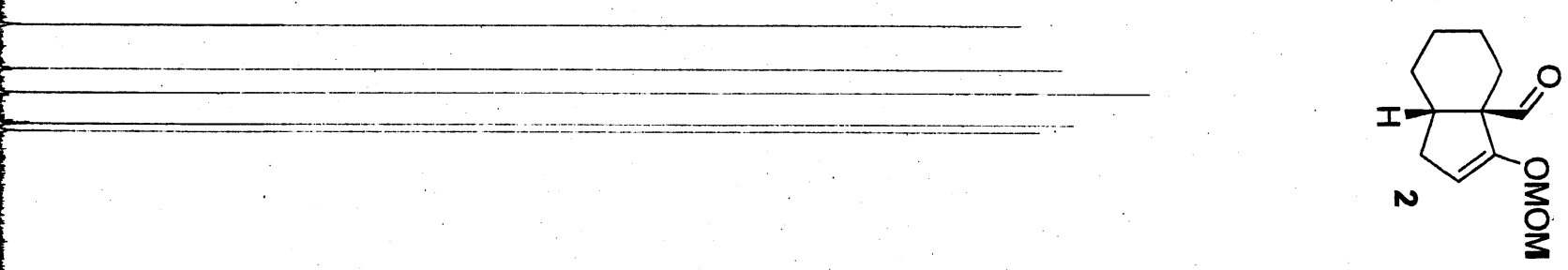

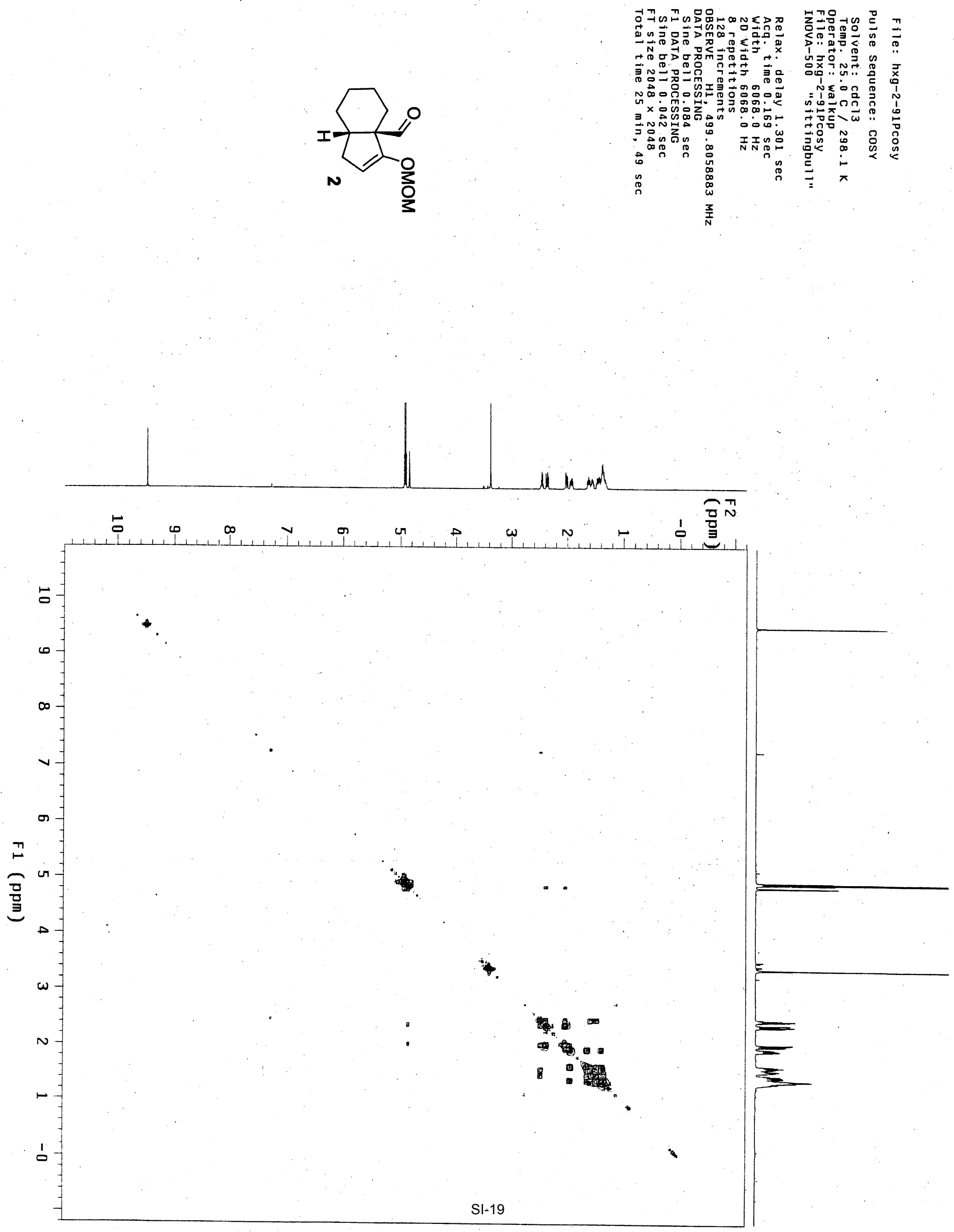


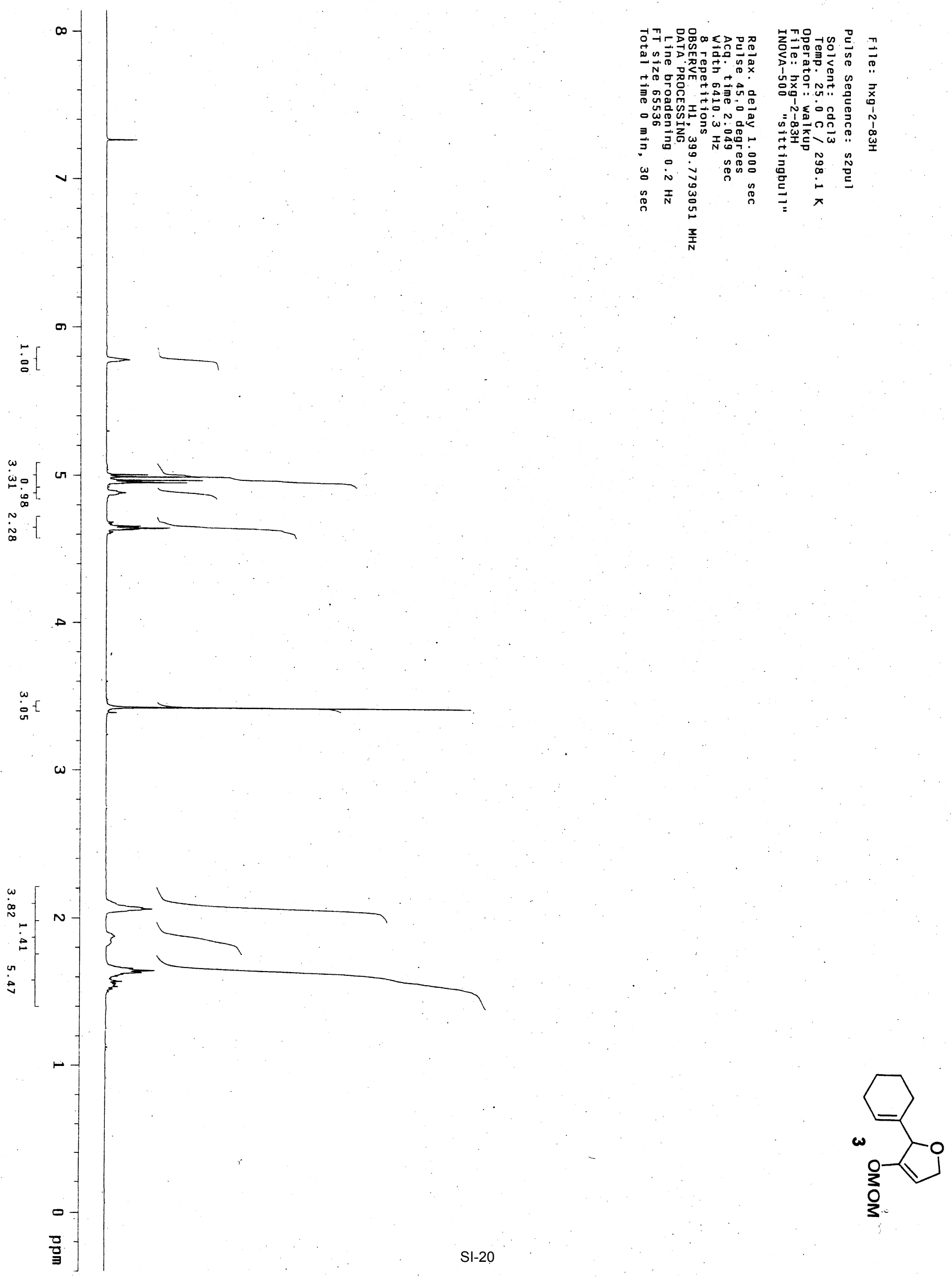



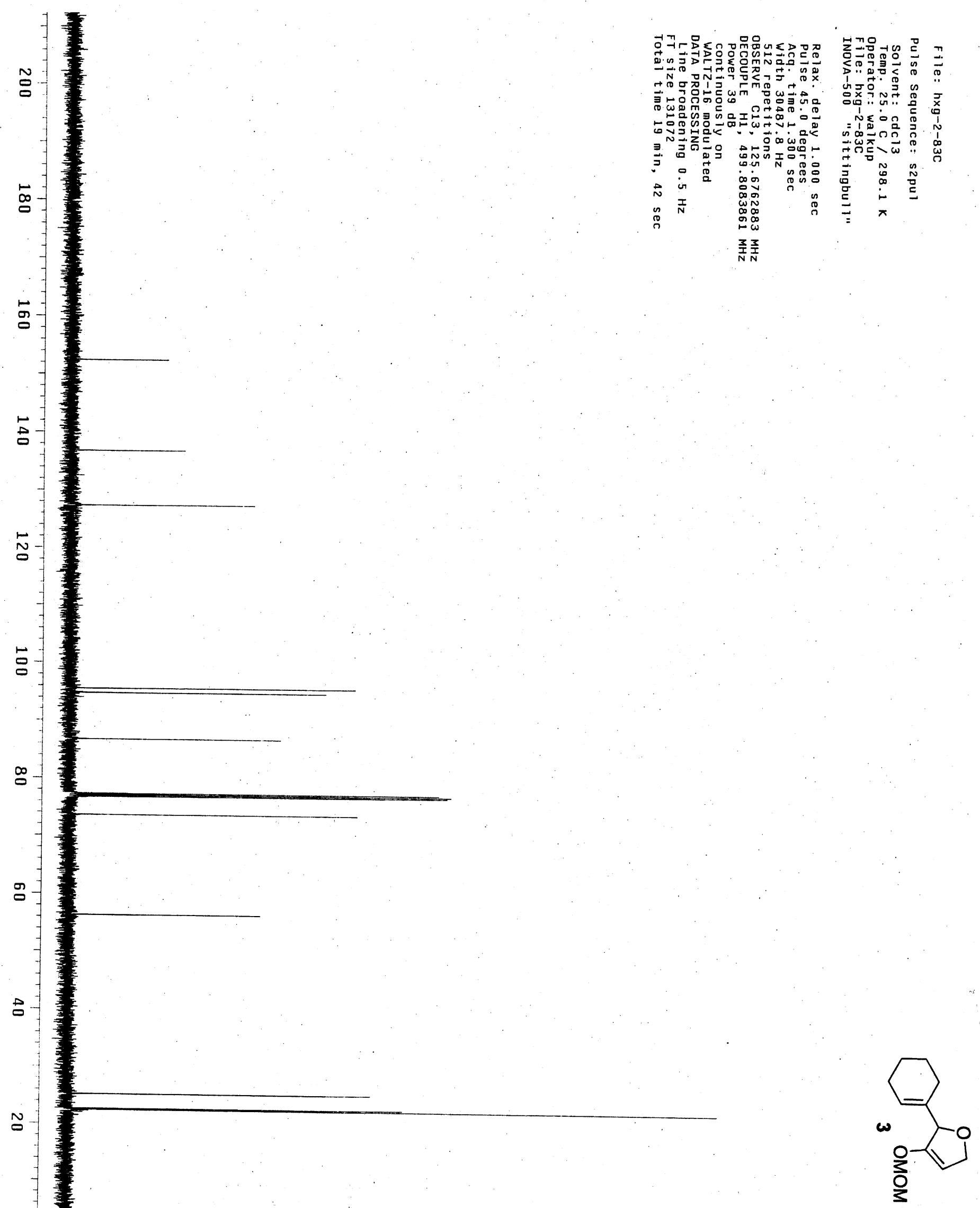

먼 


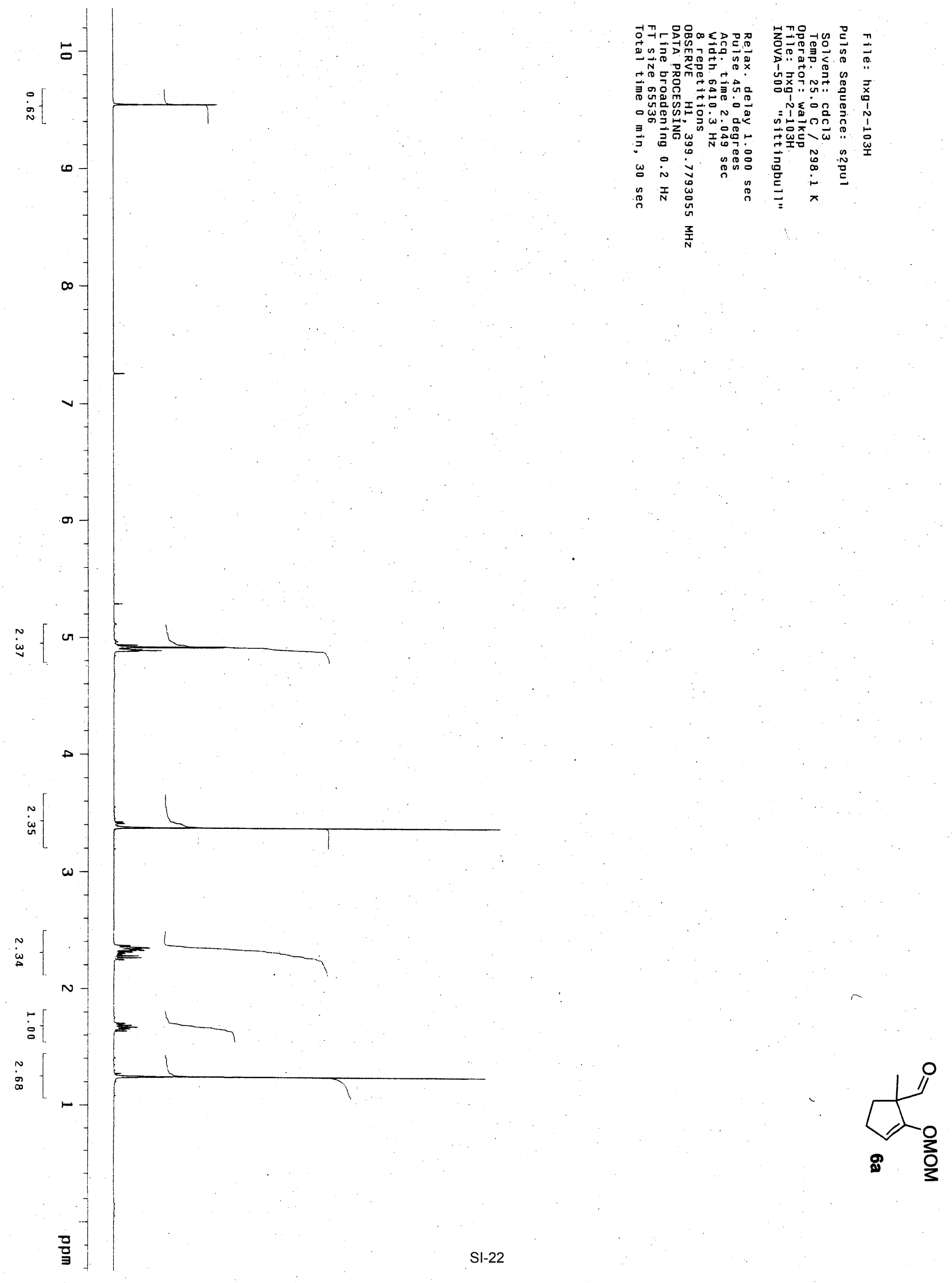



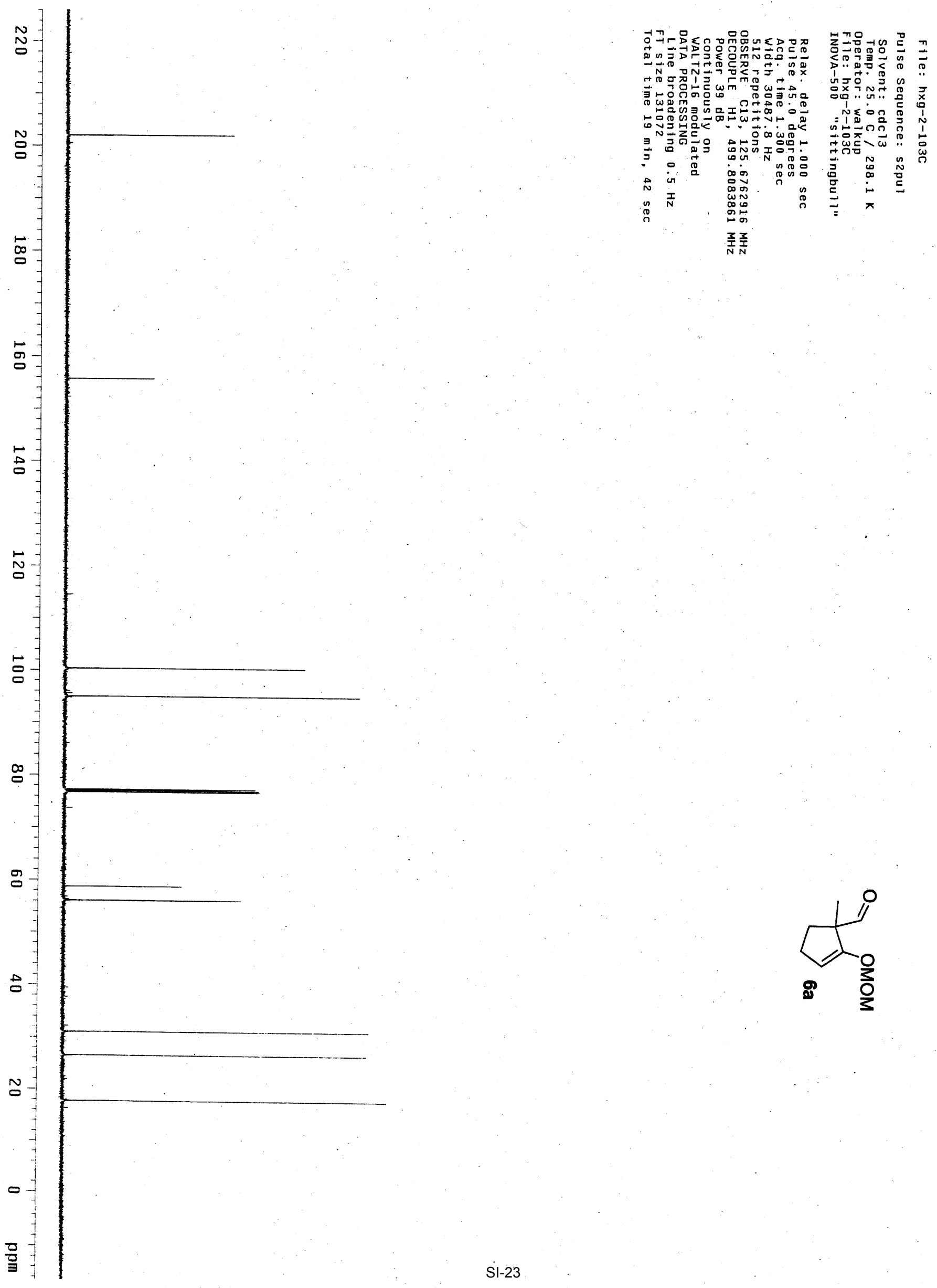


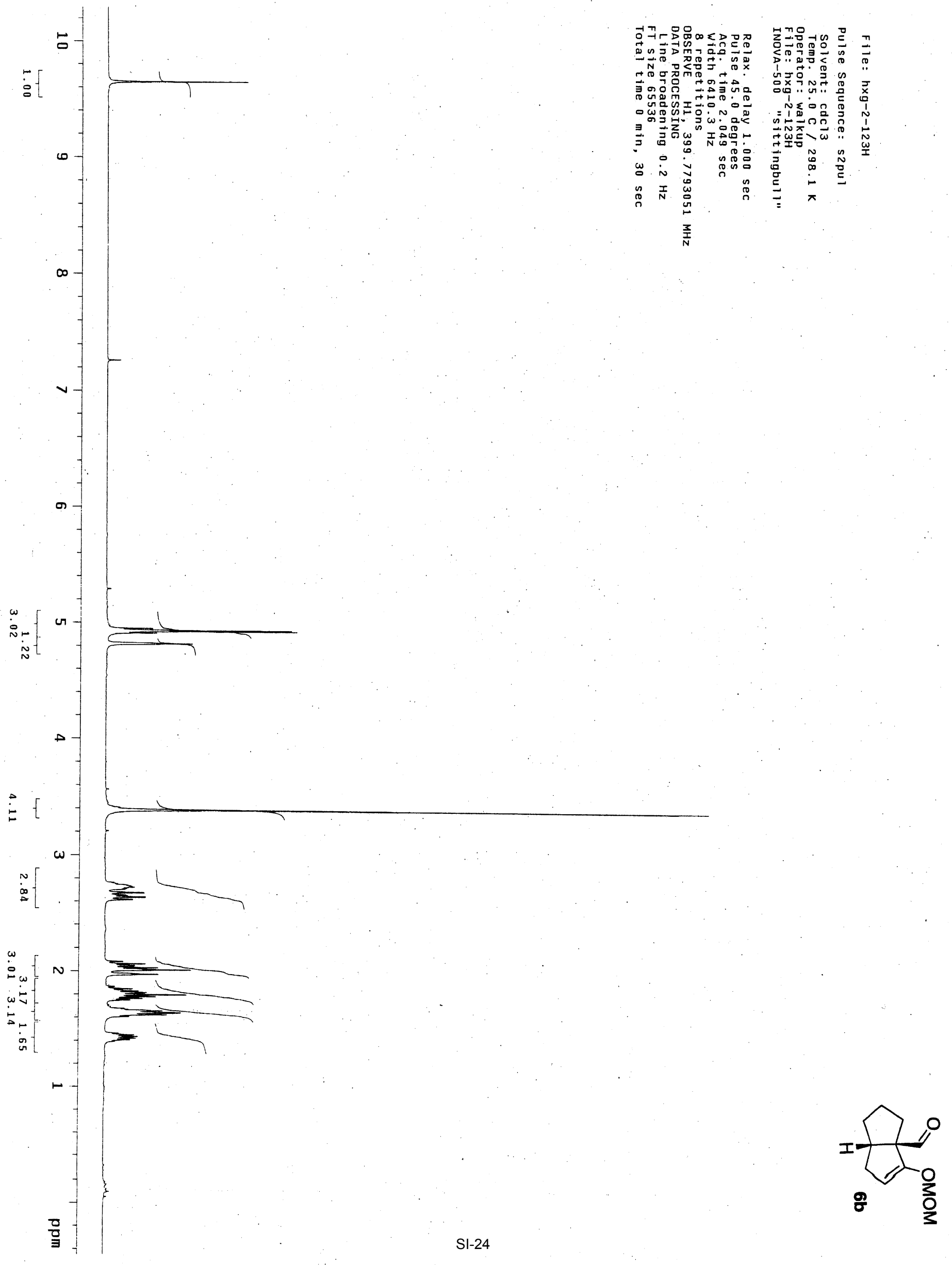



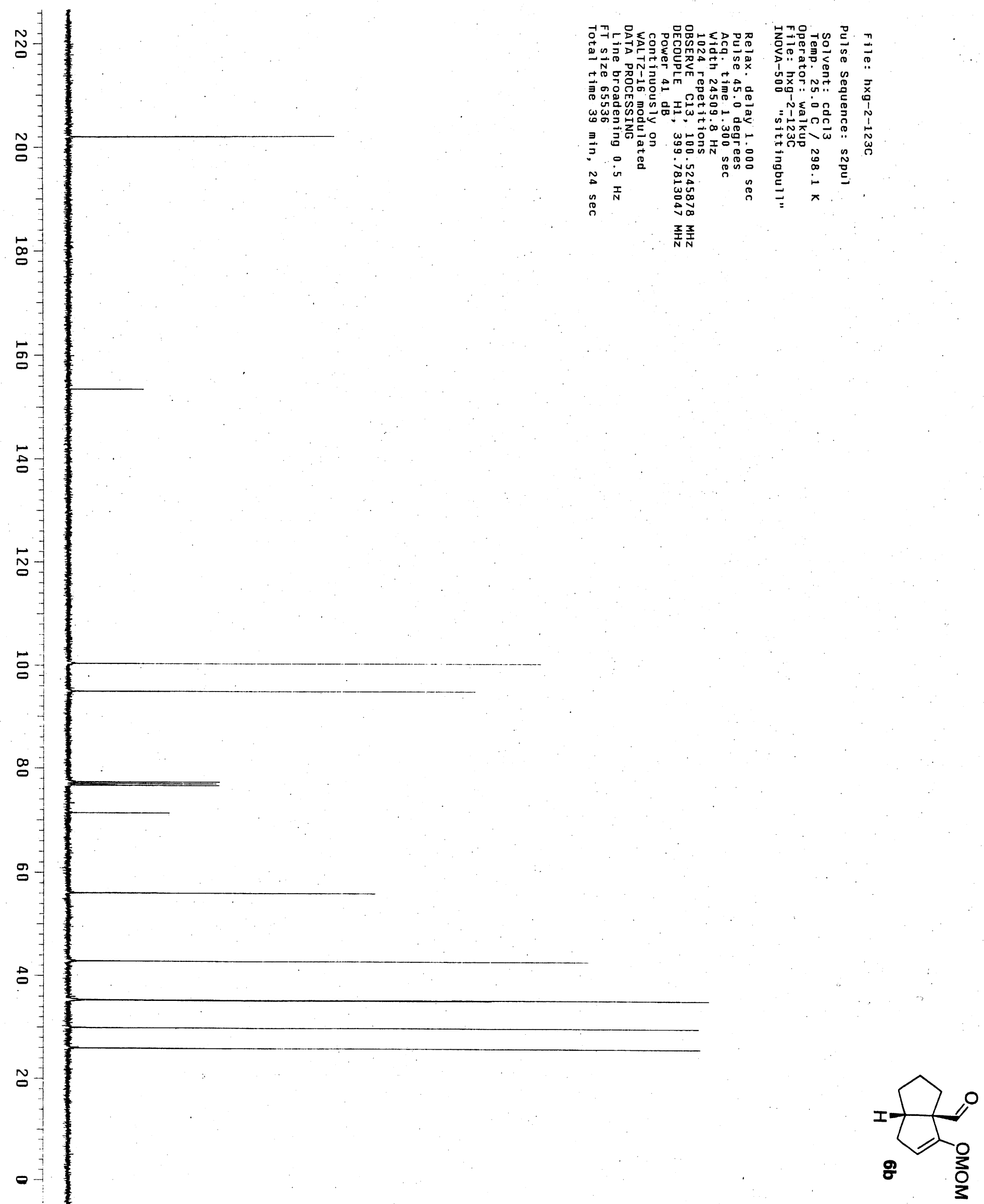


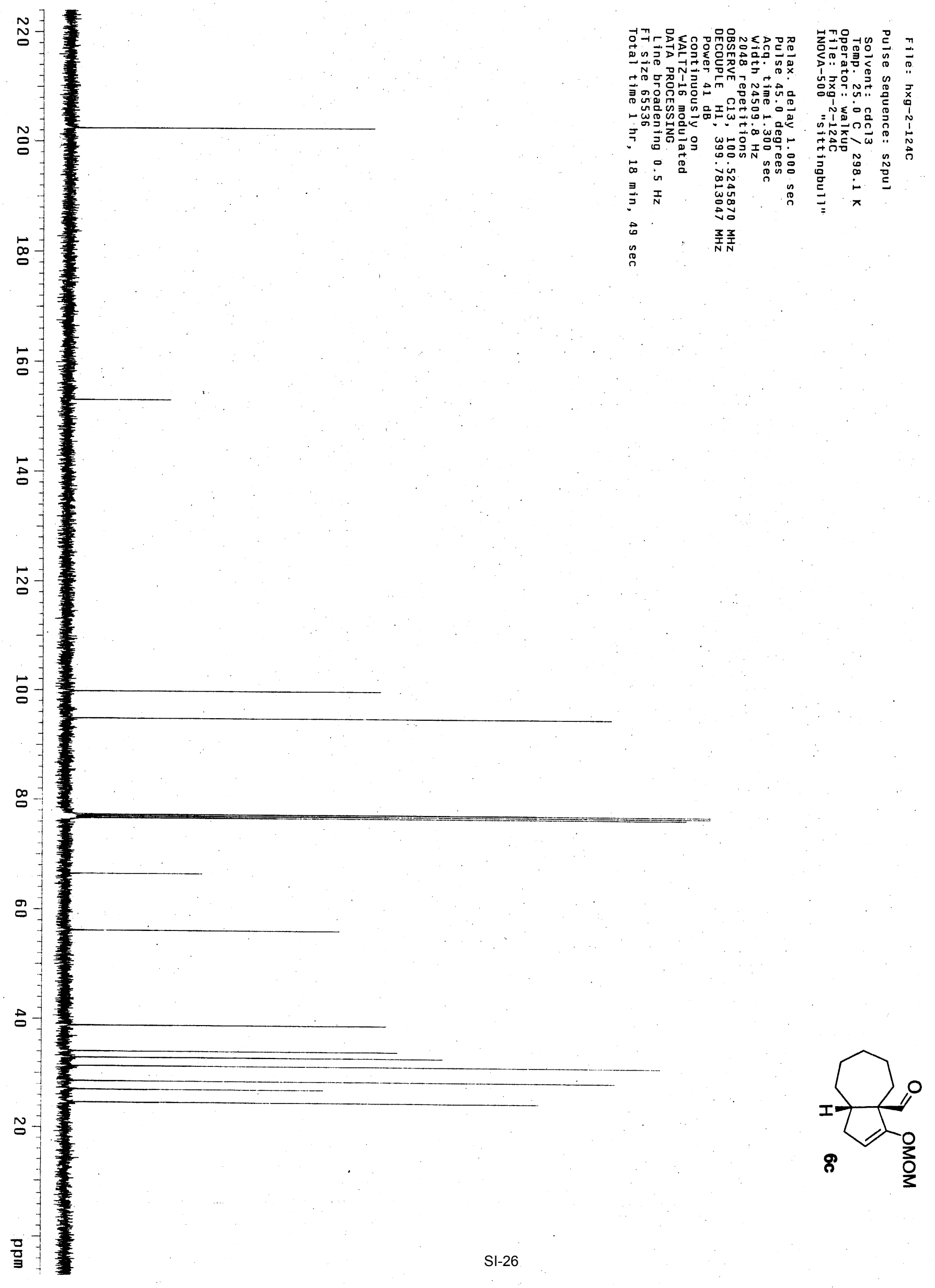




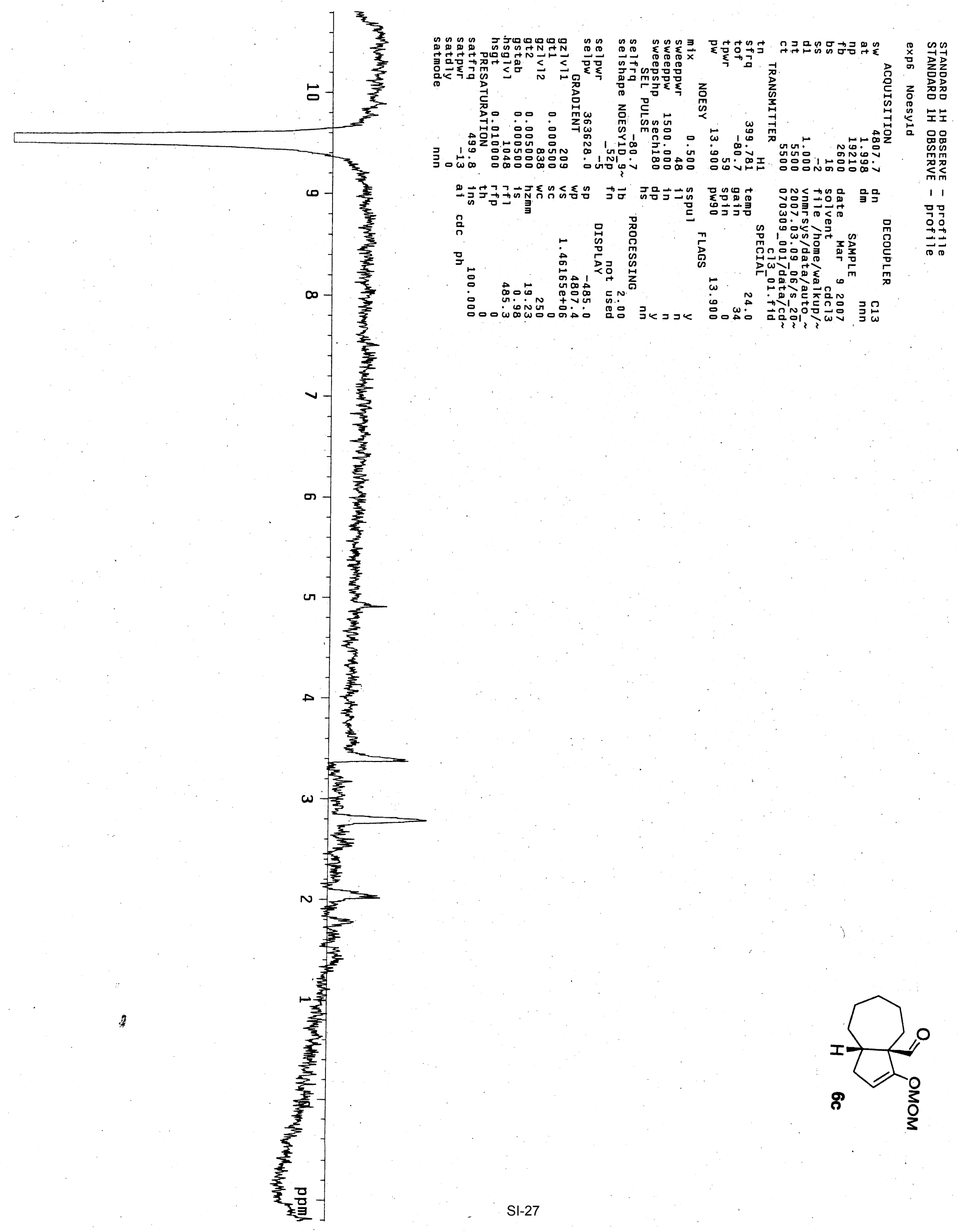



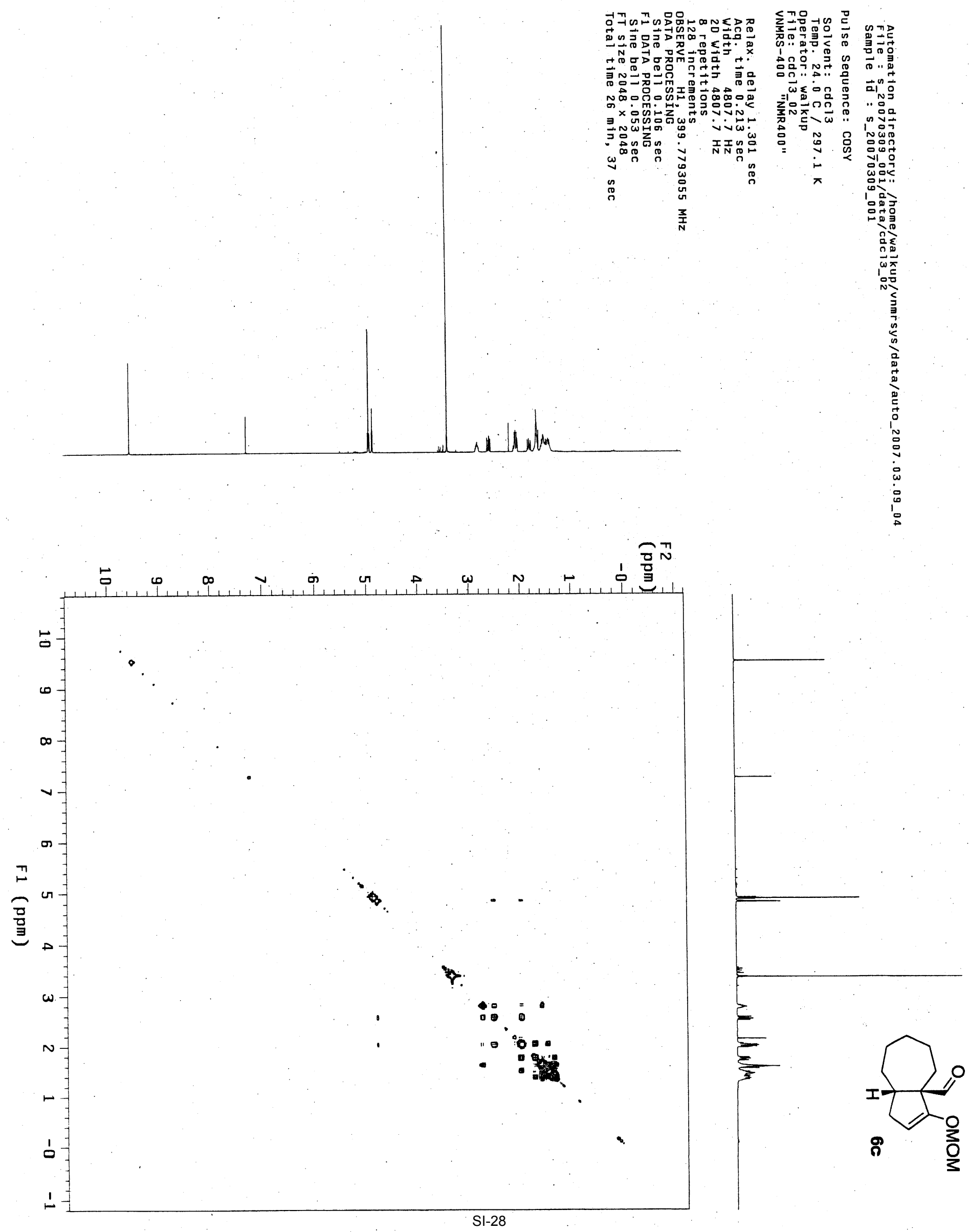


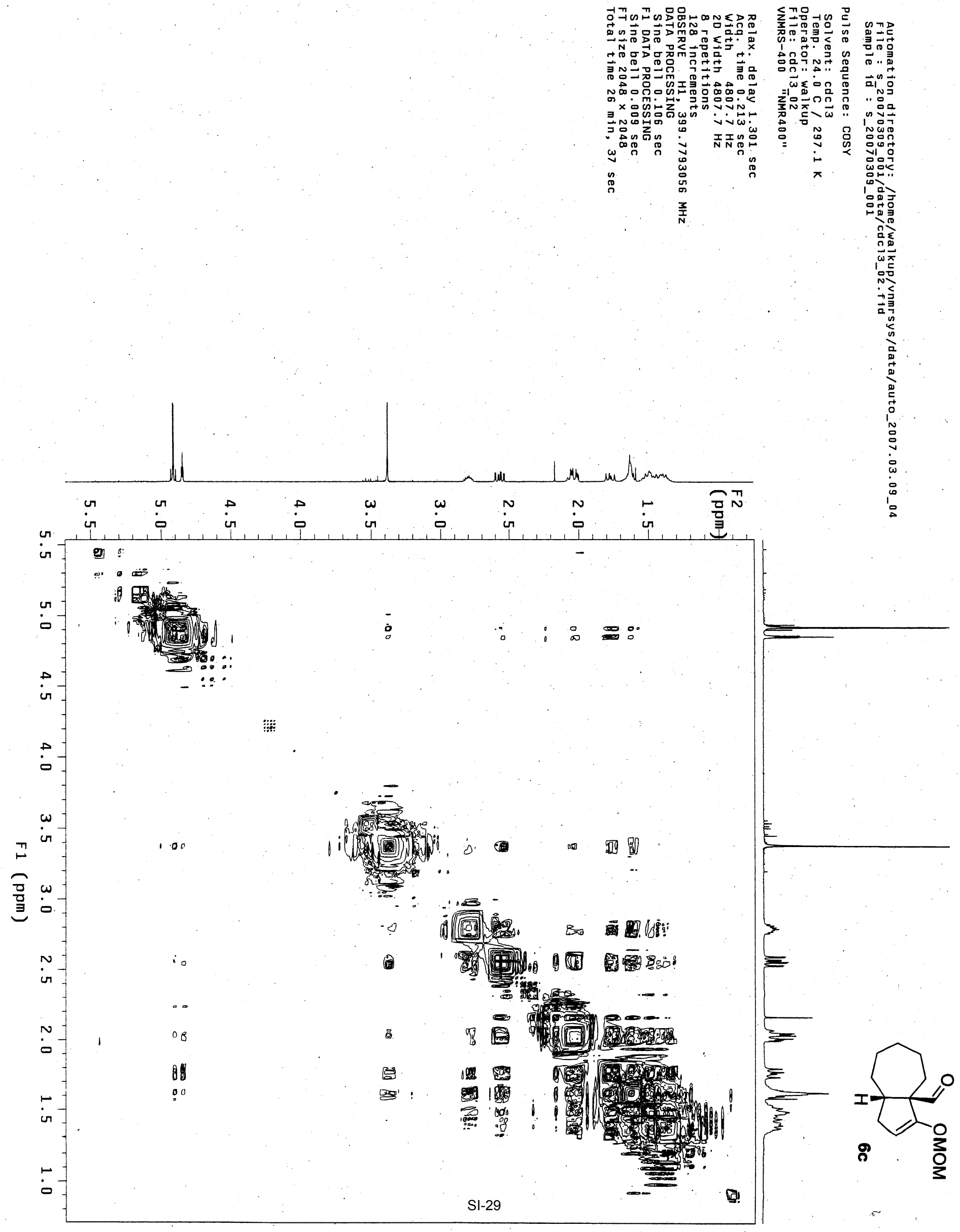




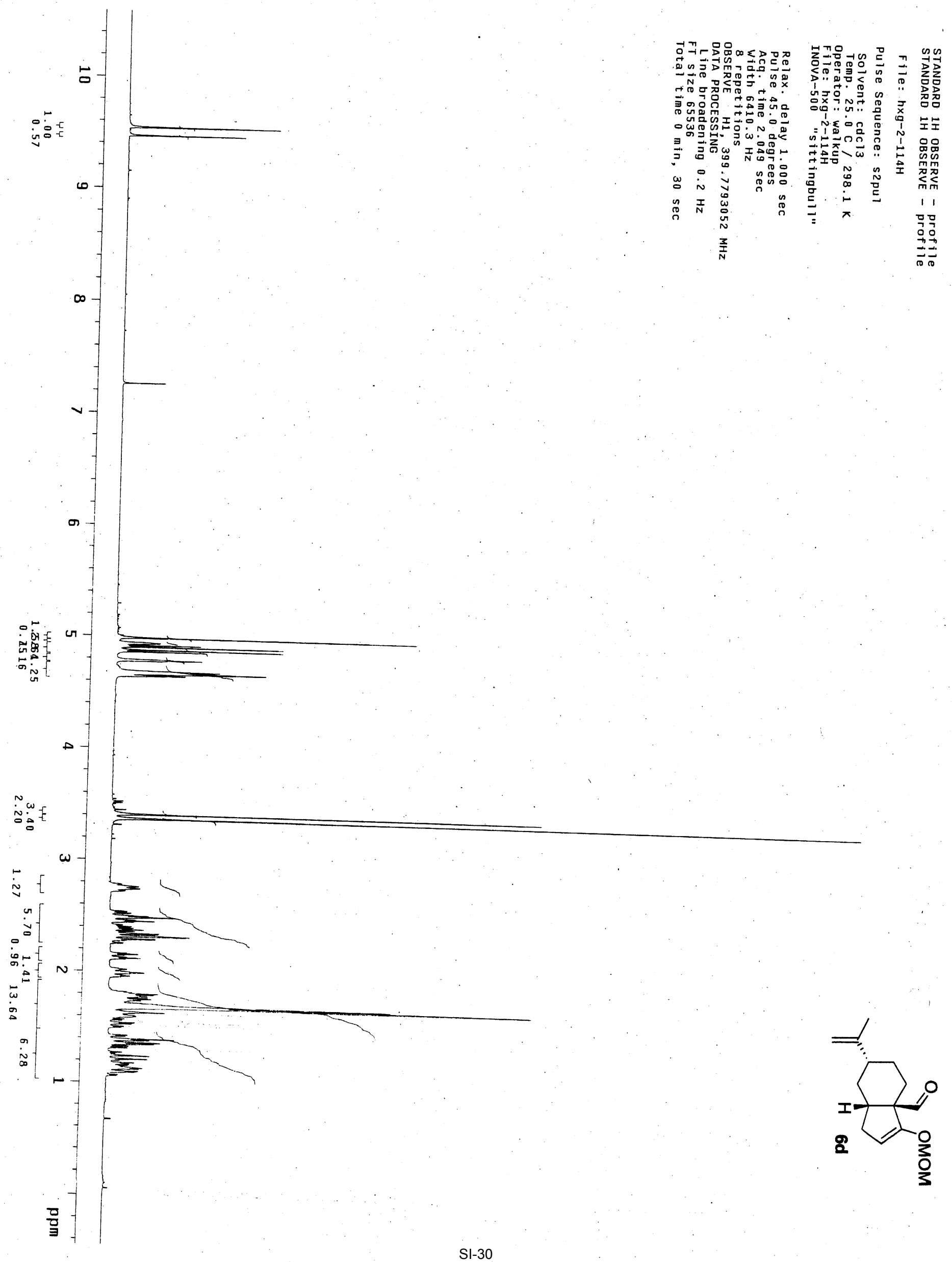



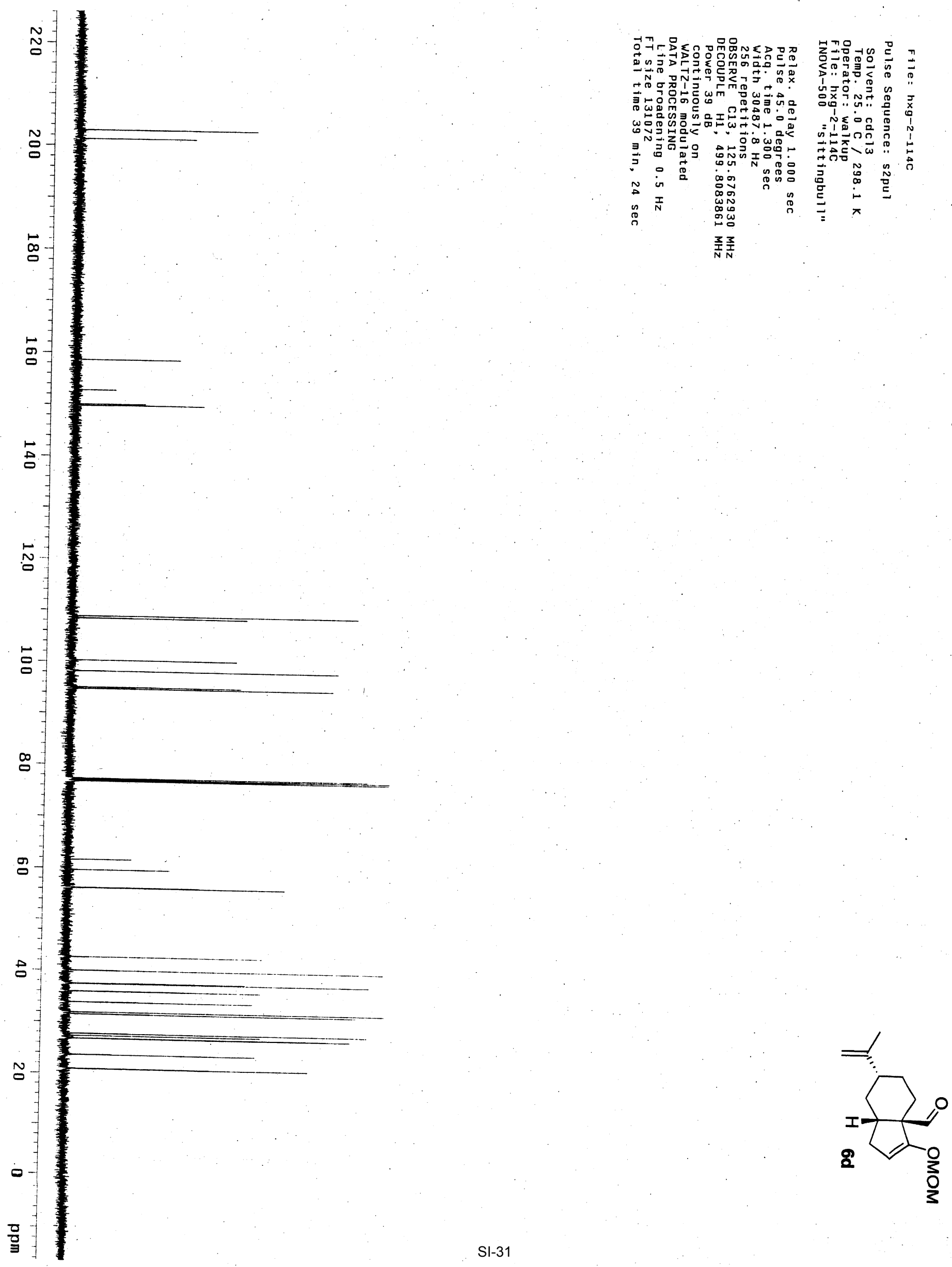


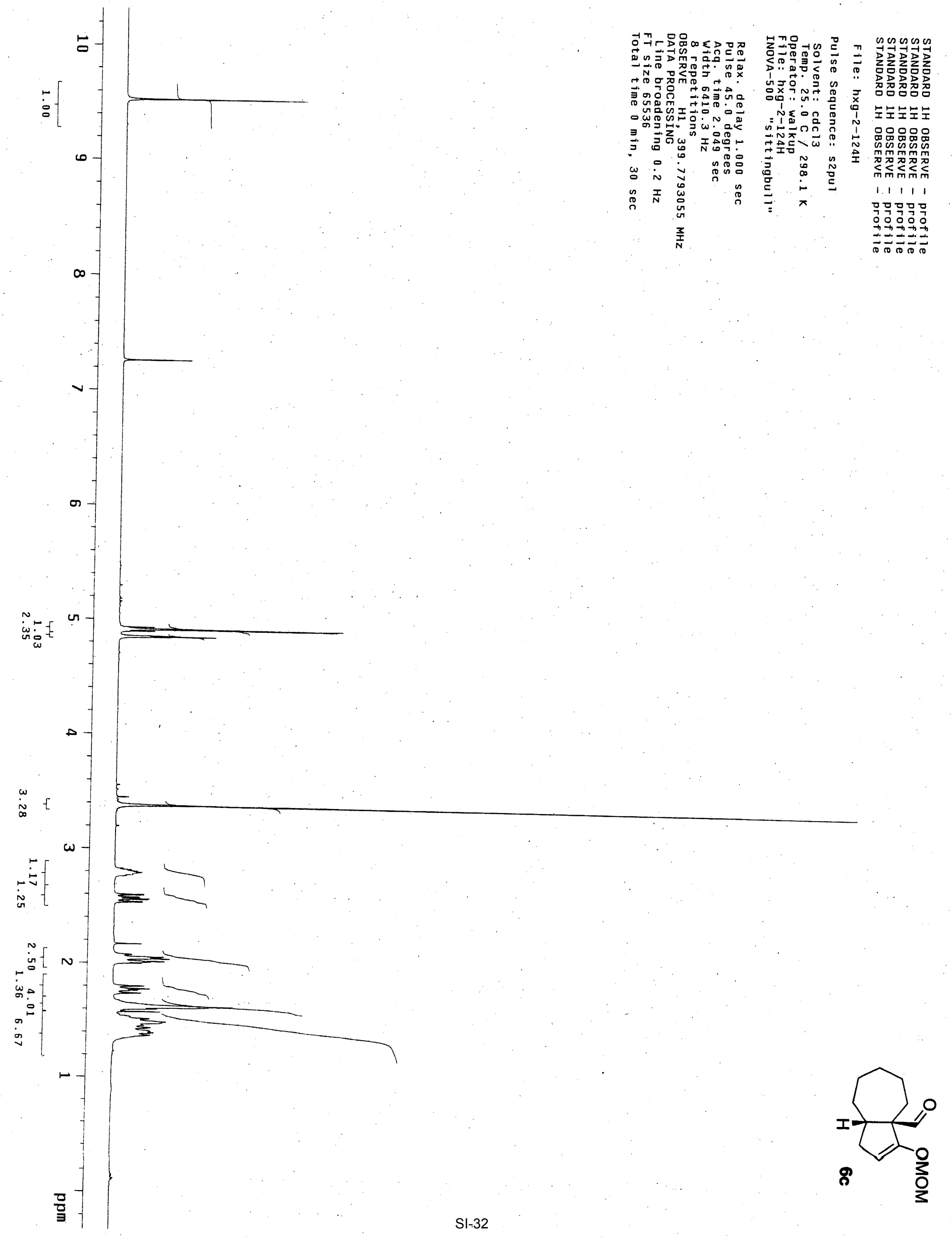



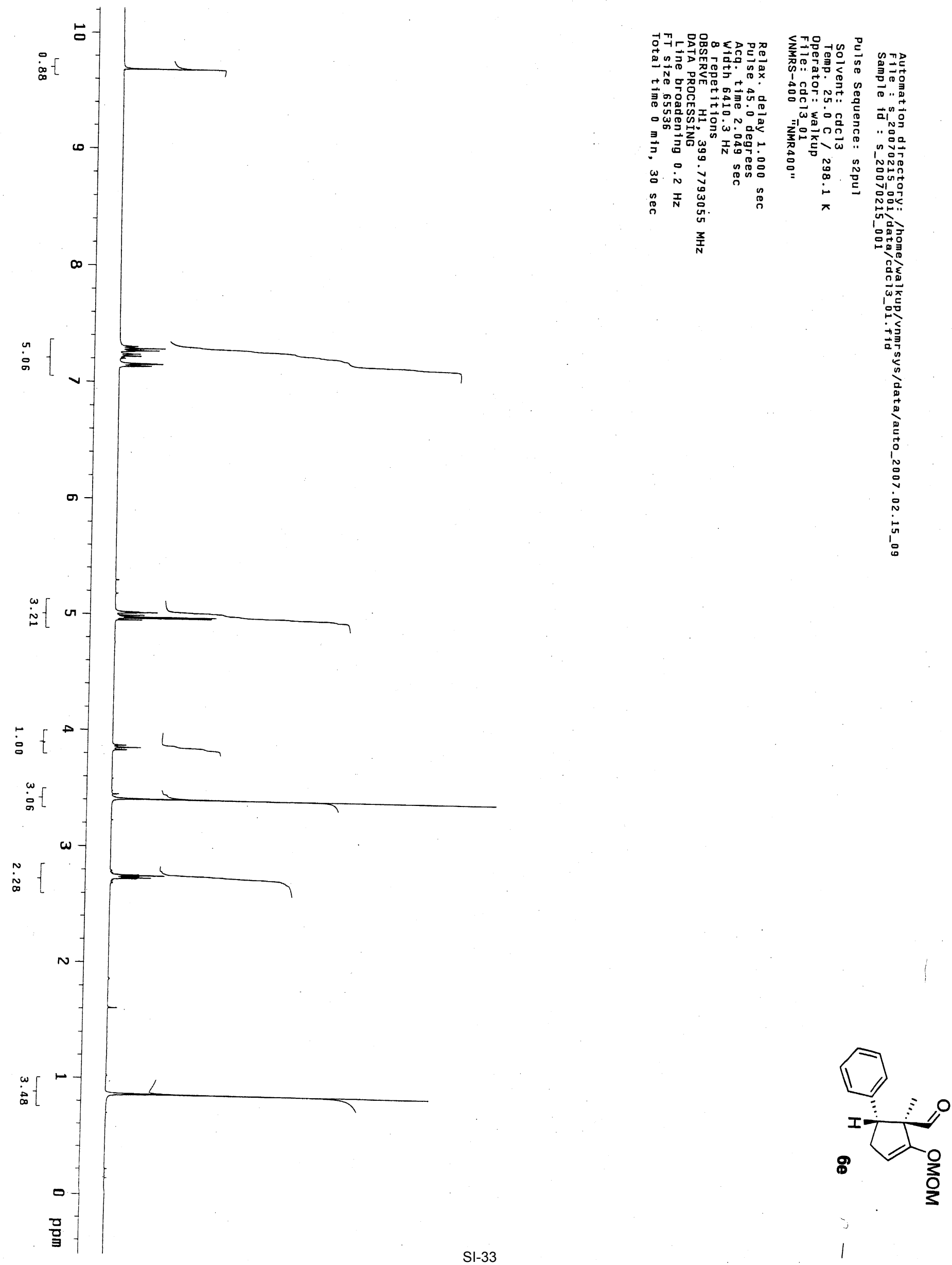

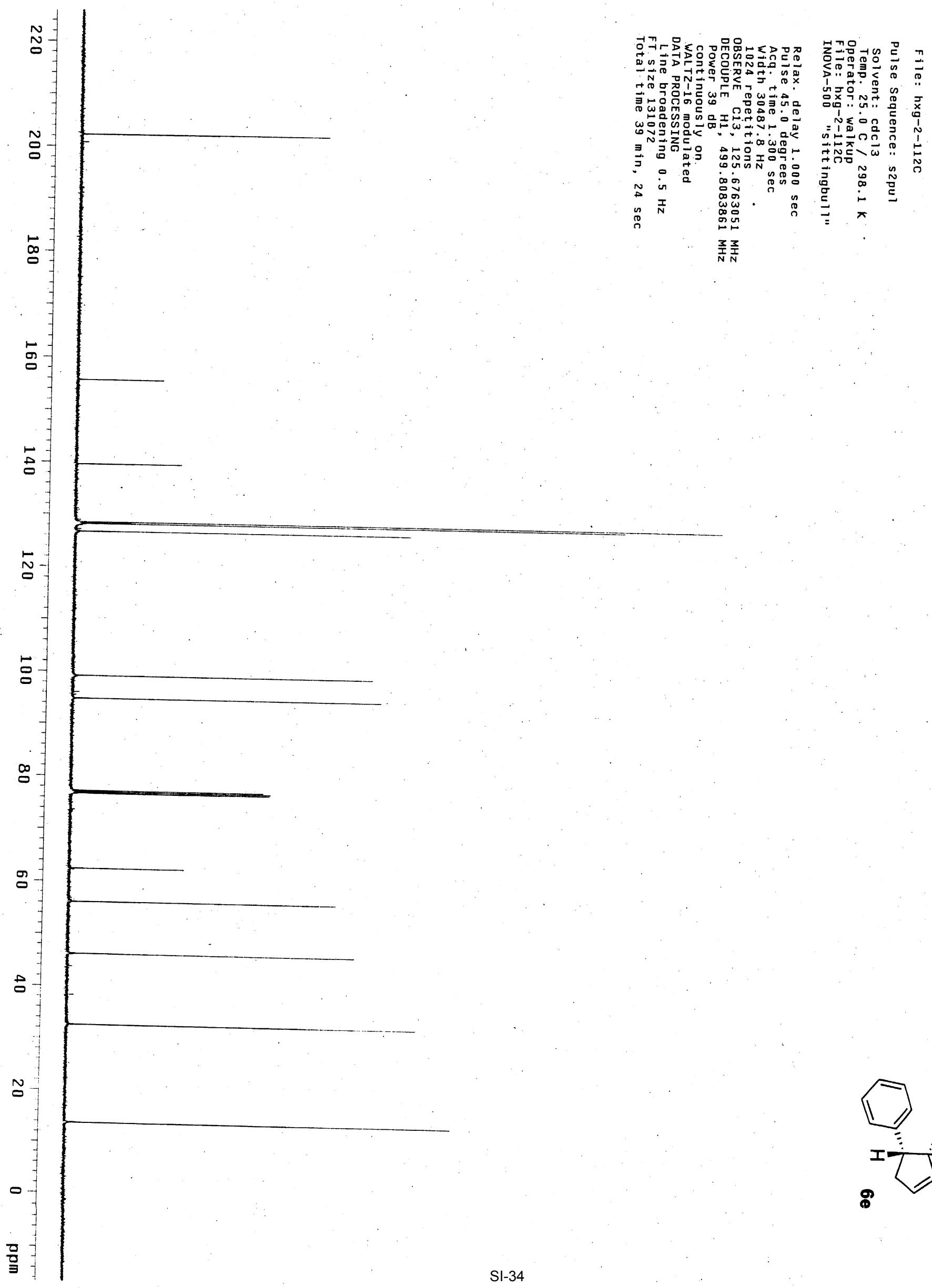


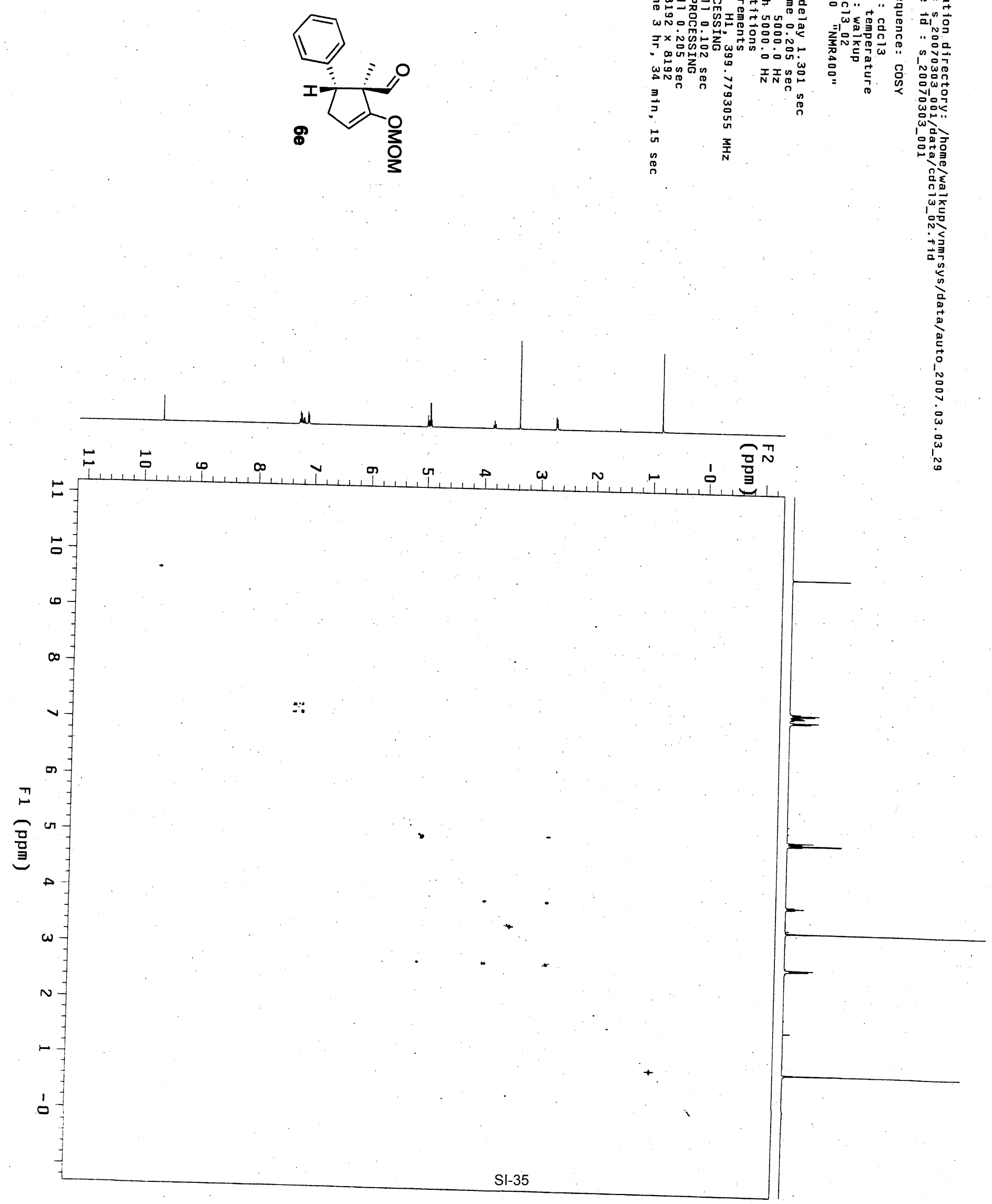




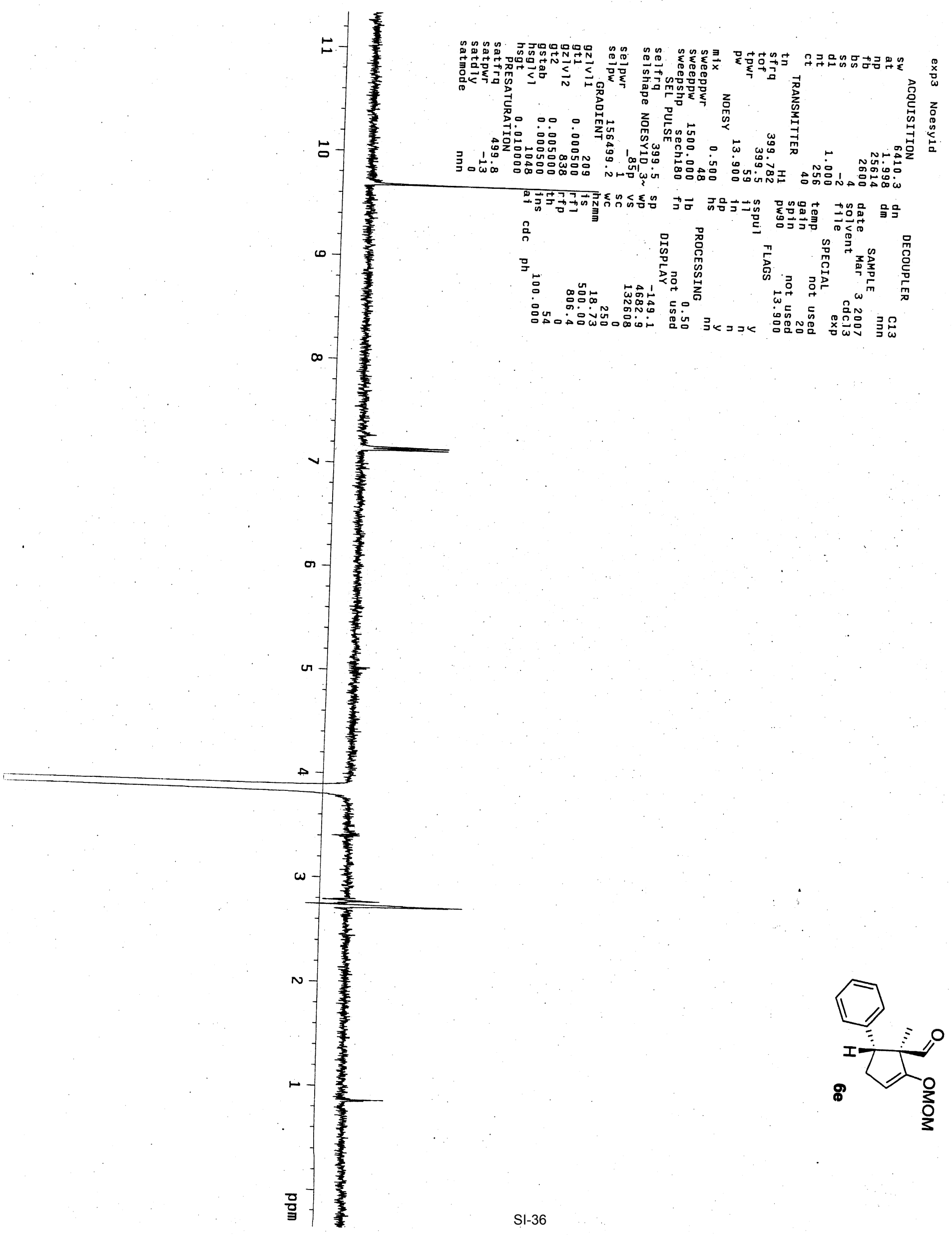




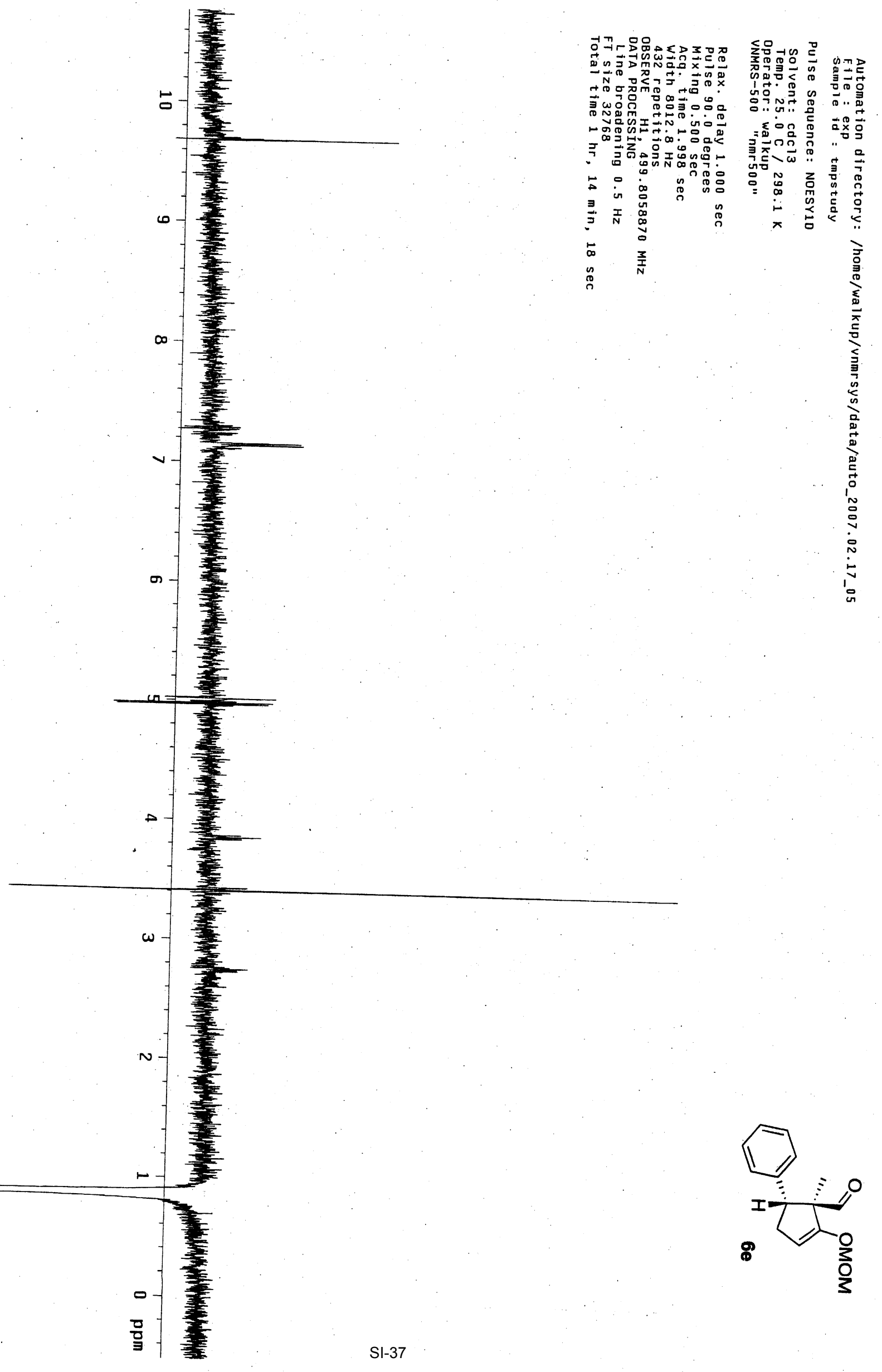




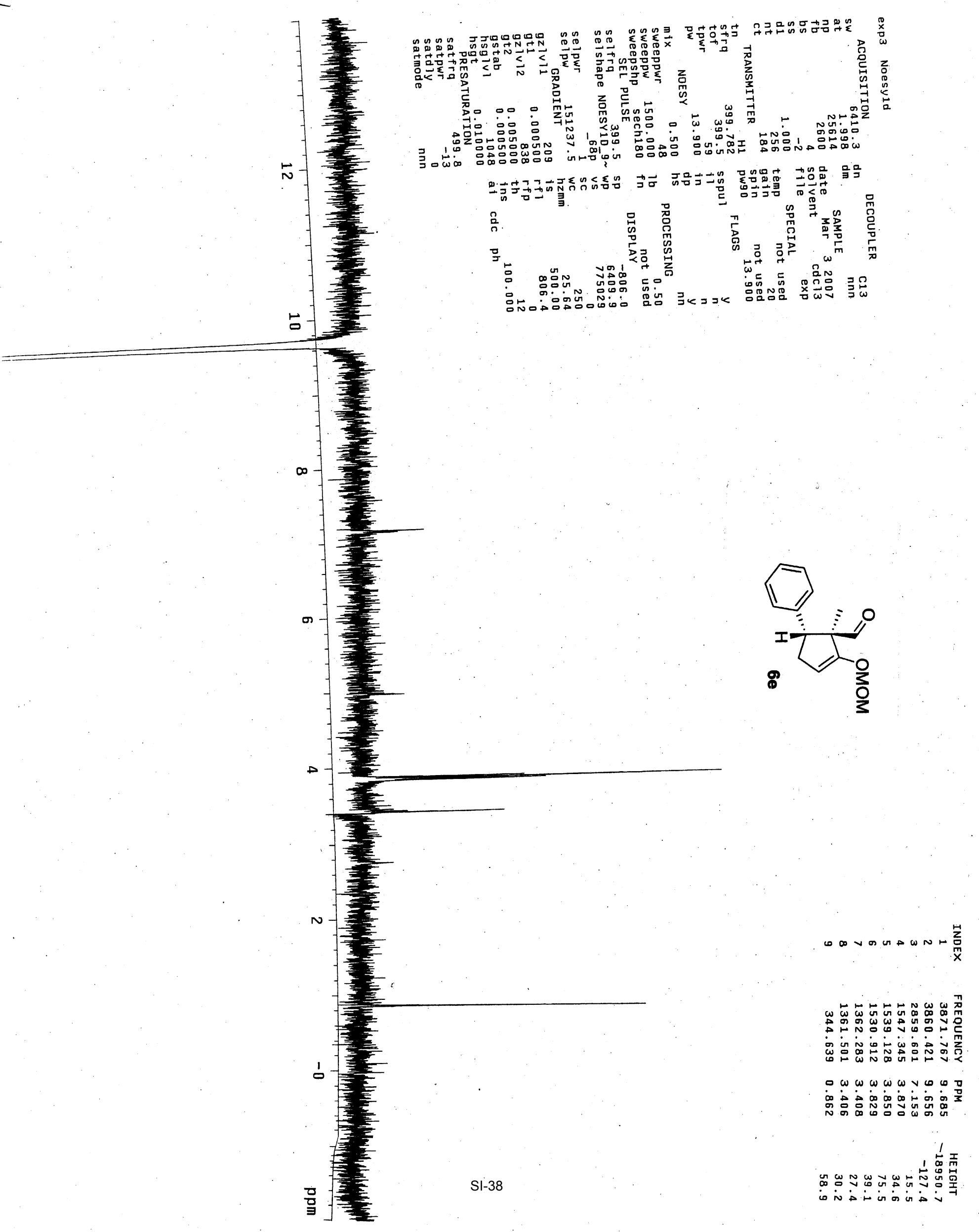




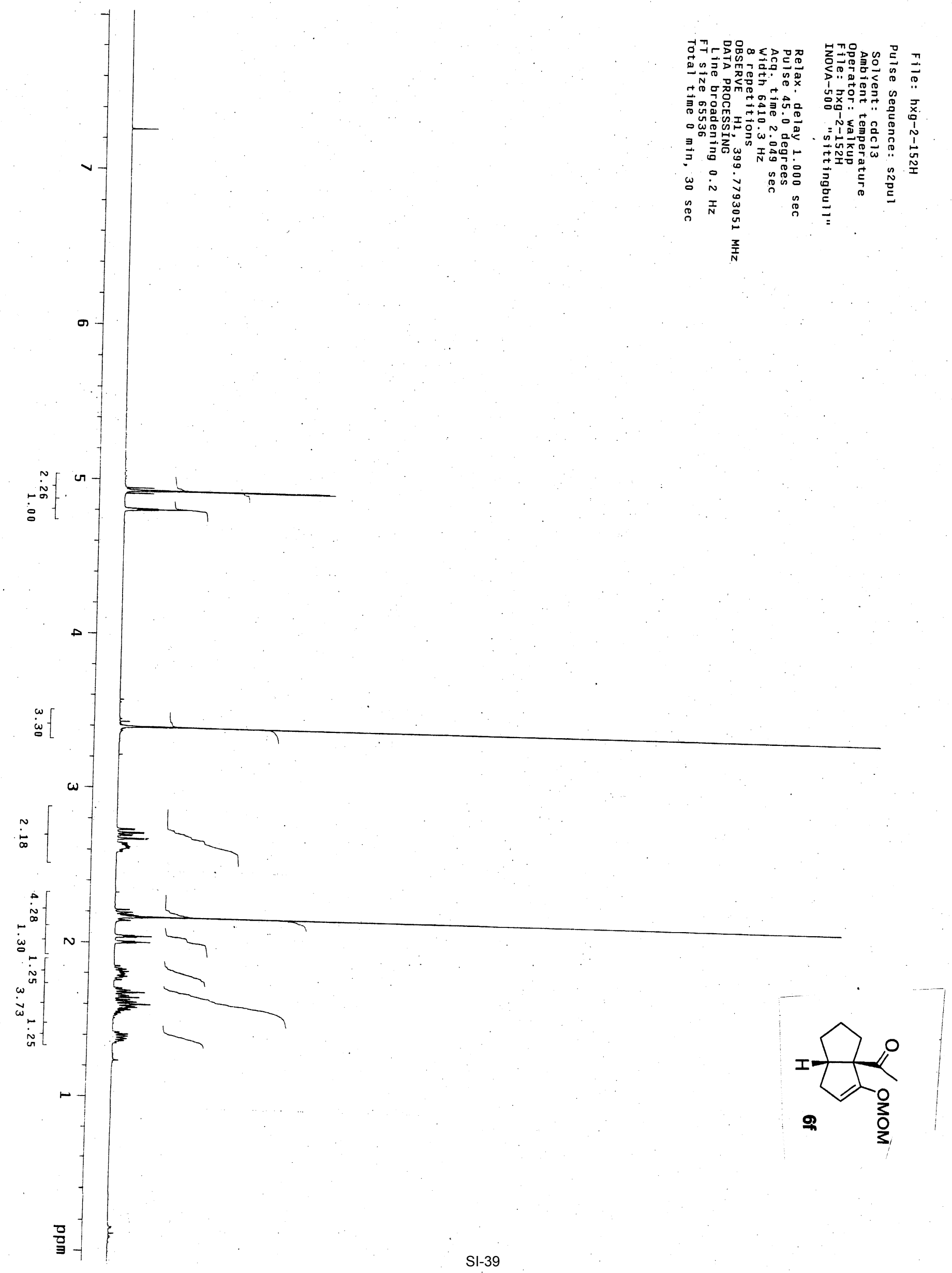




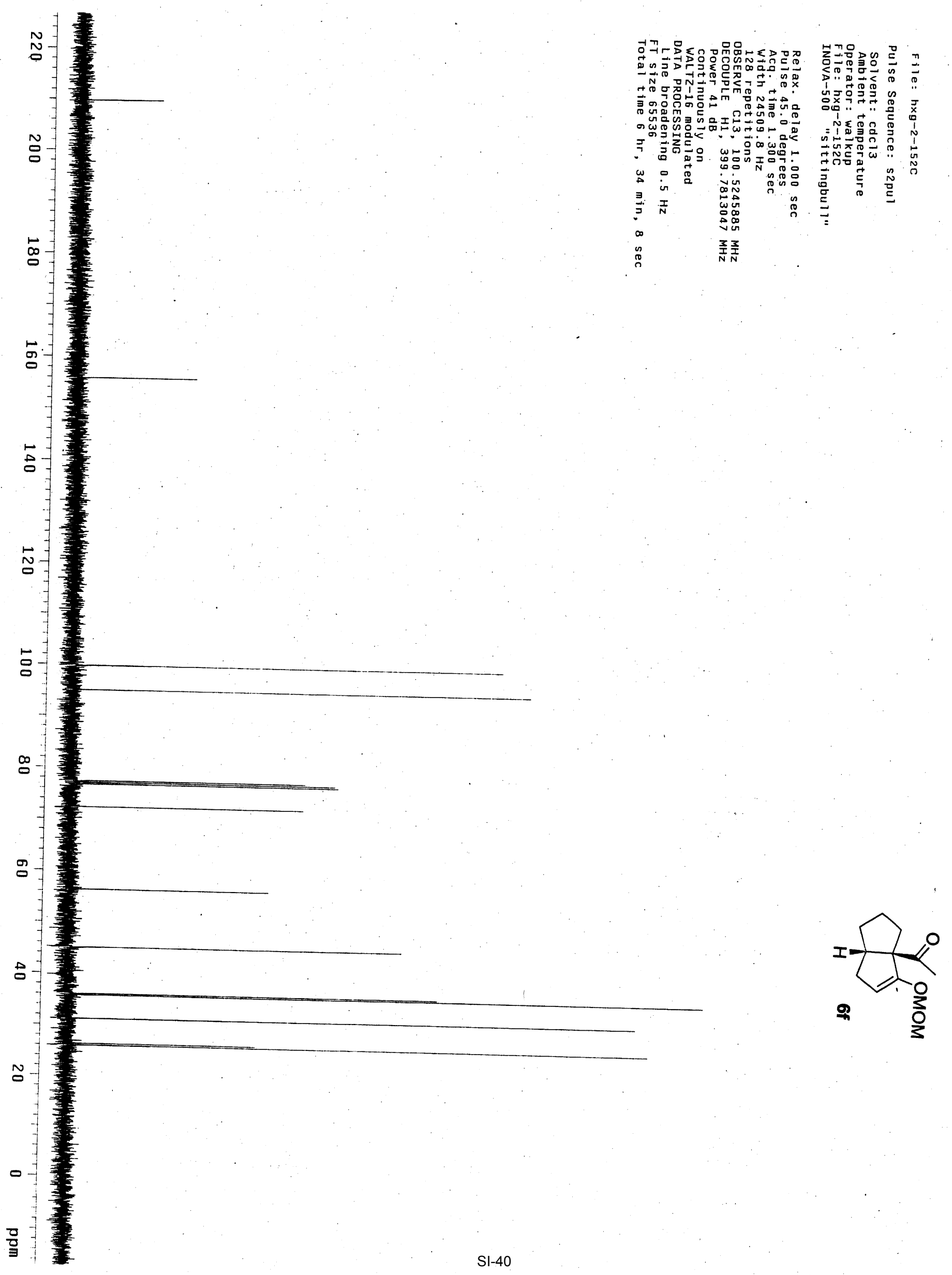




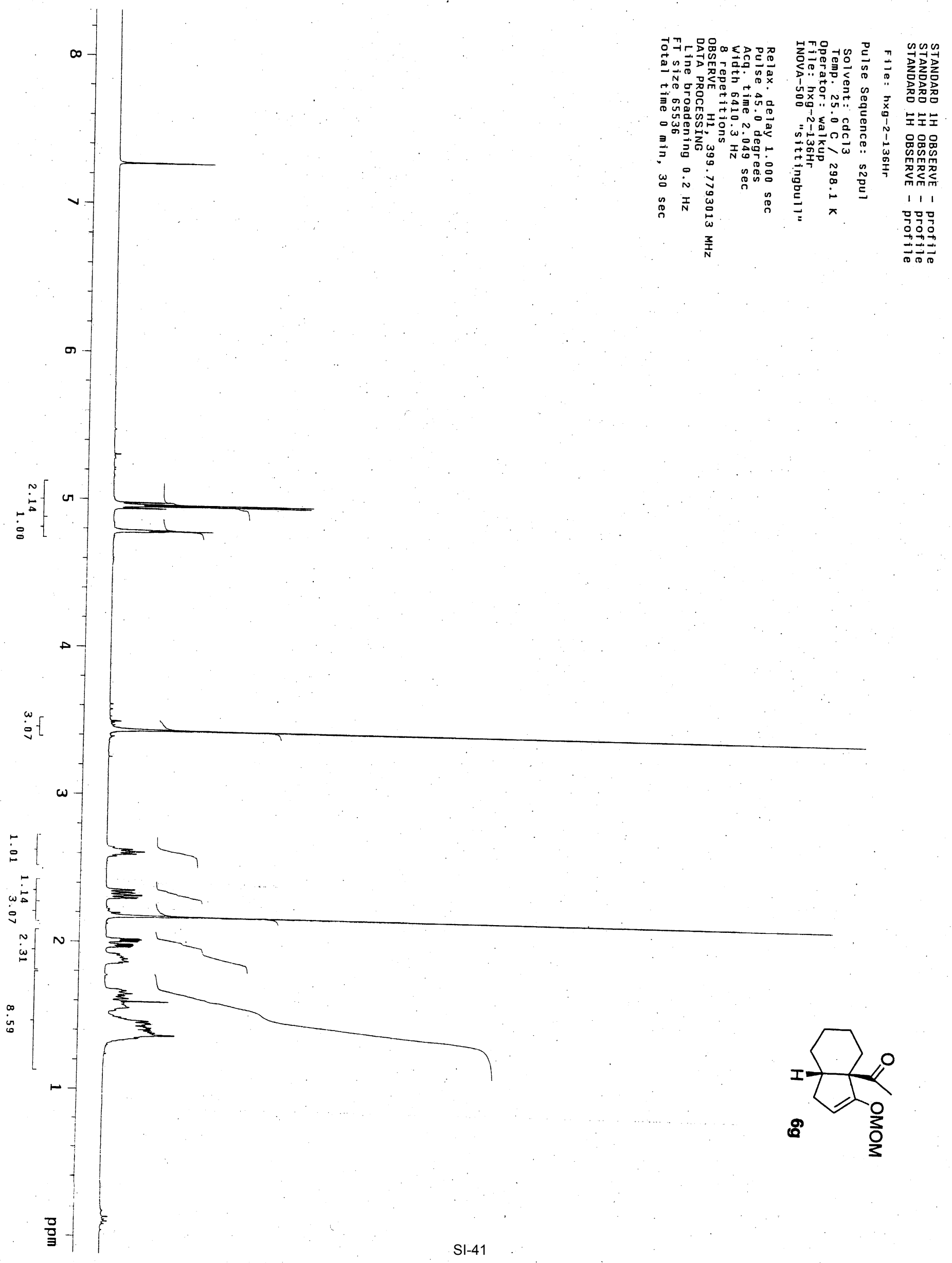



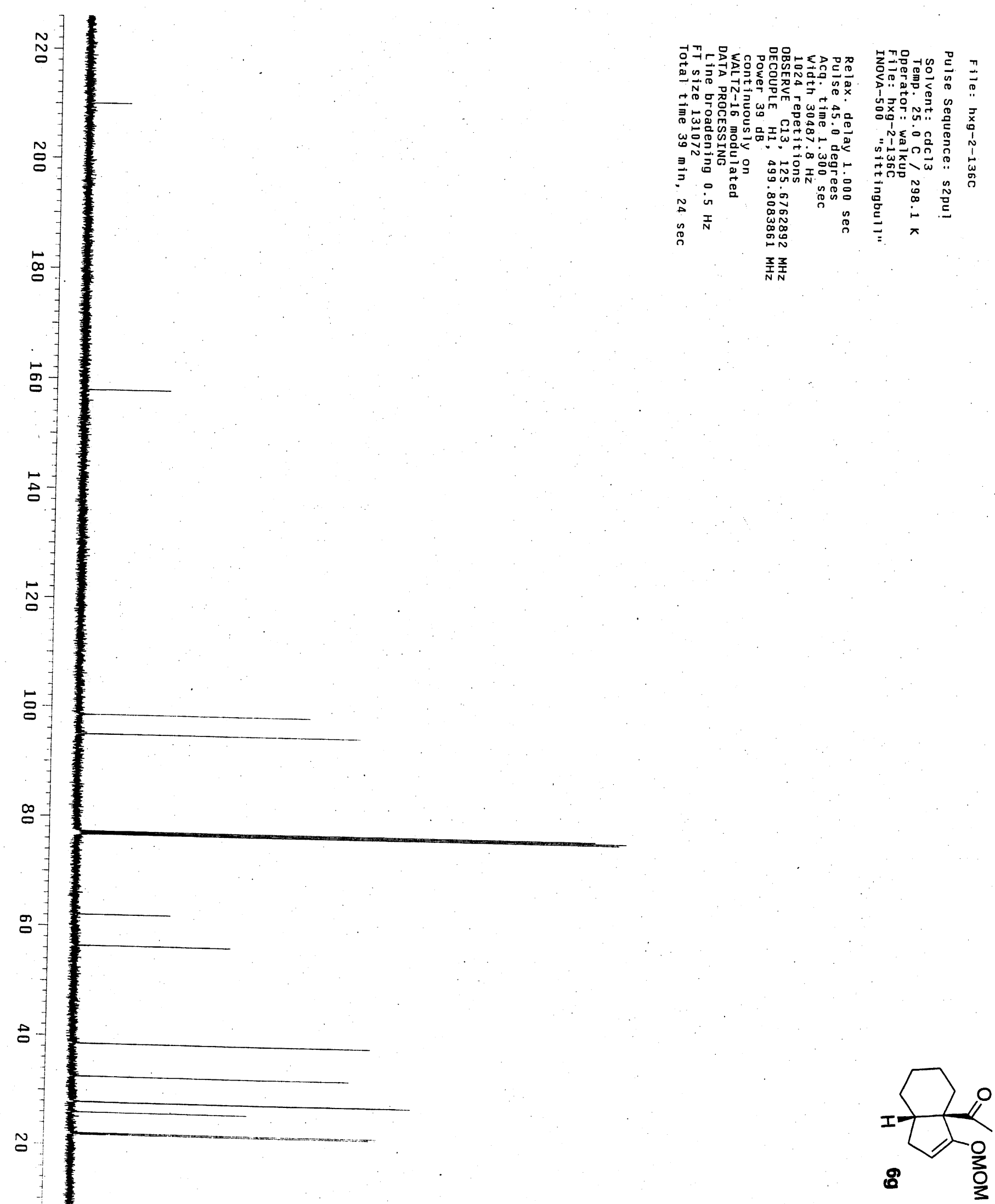

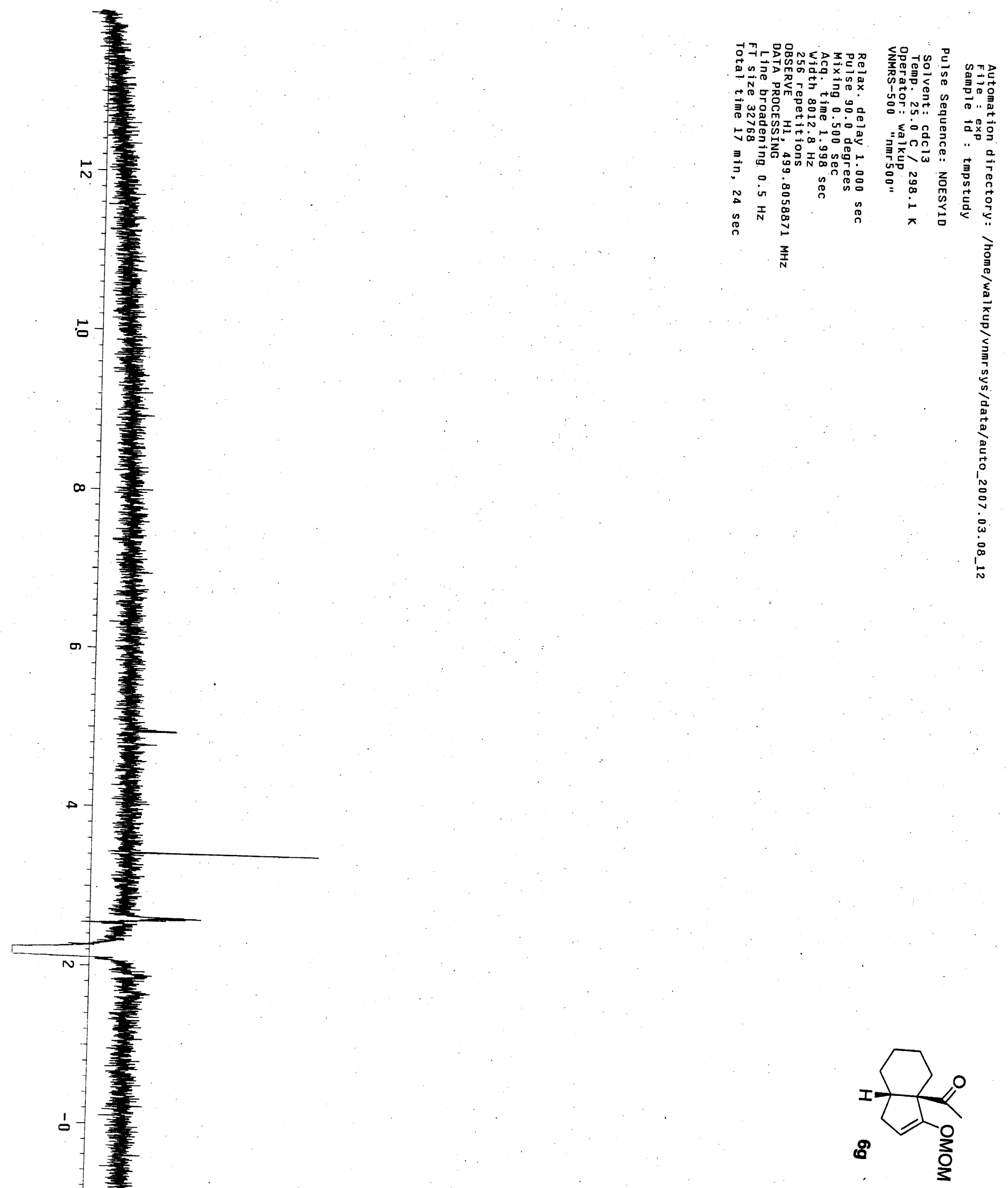

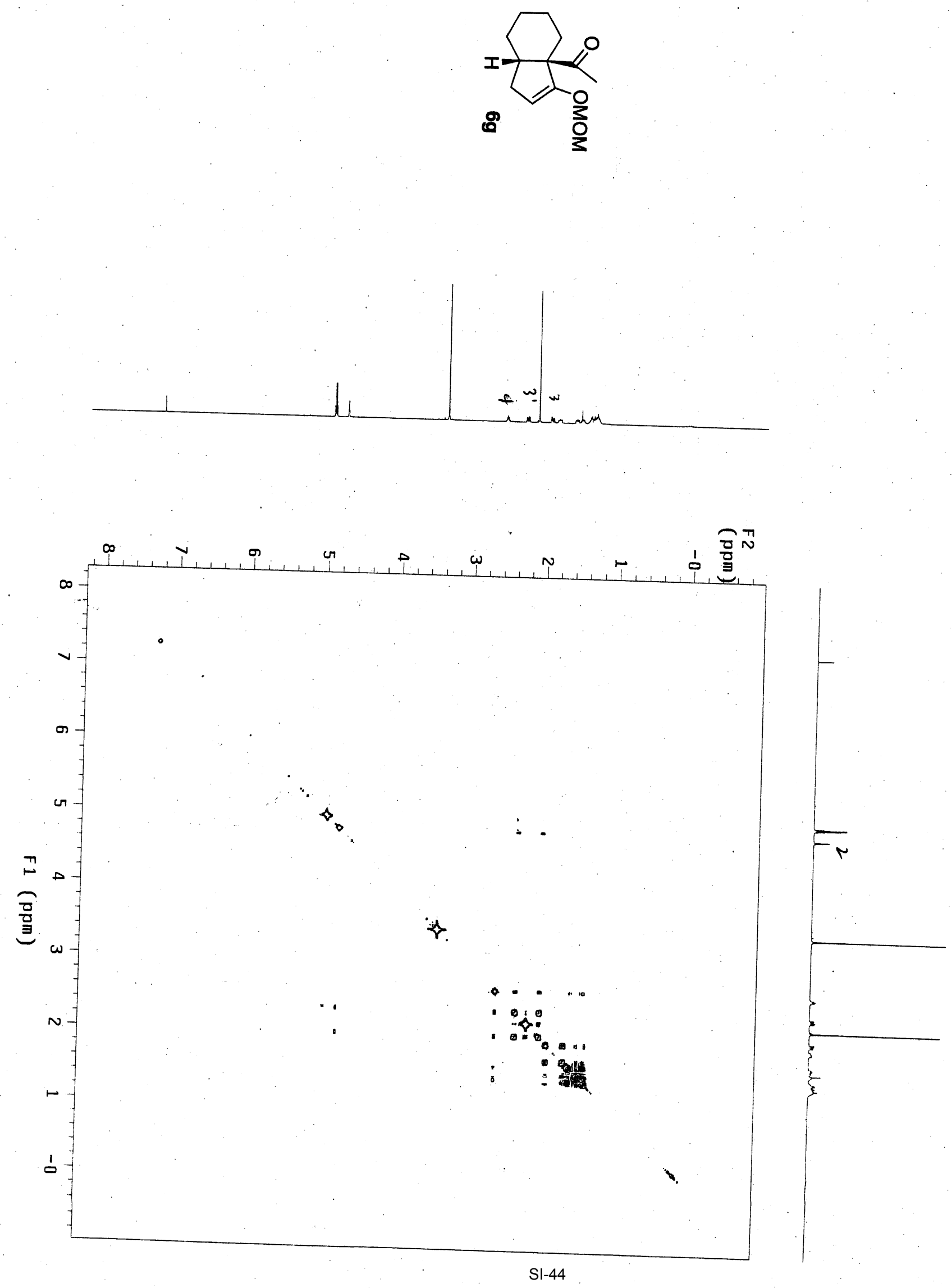


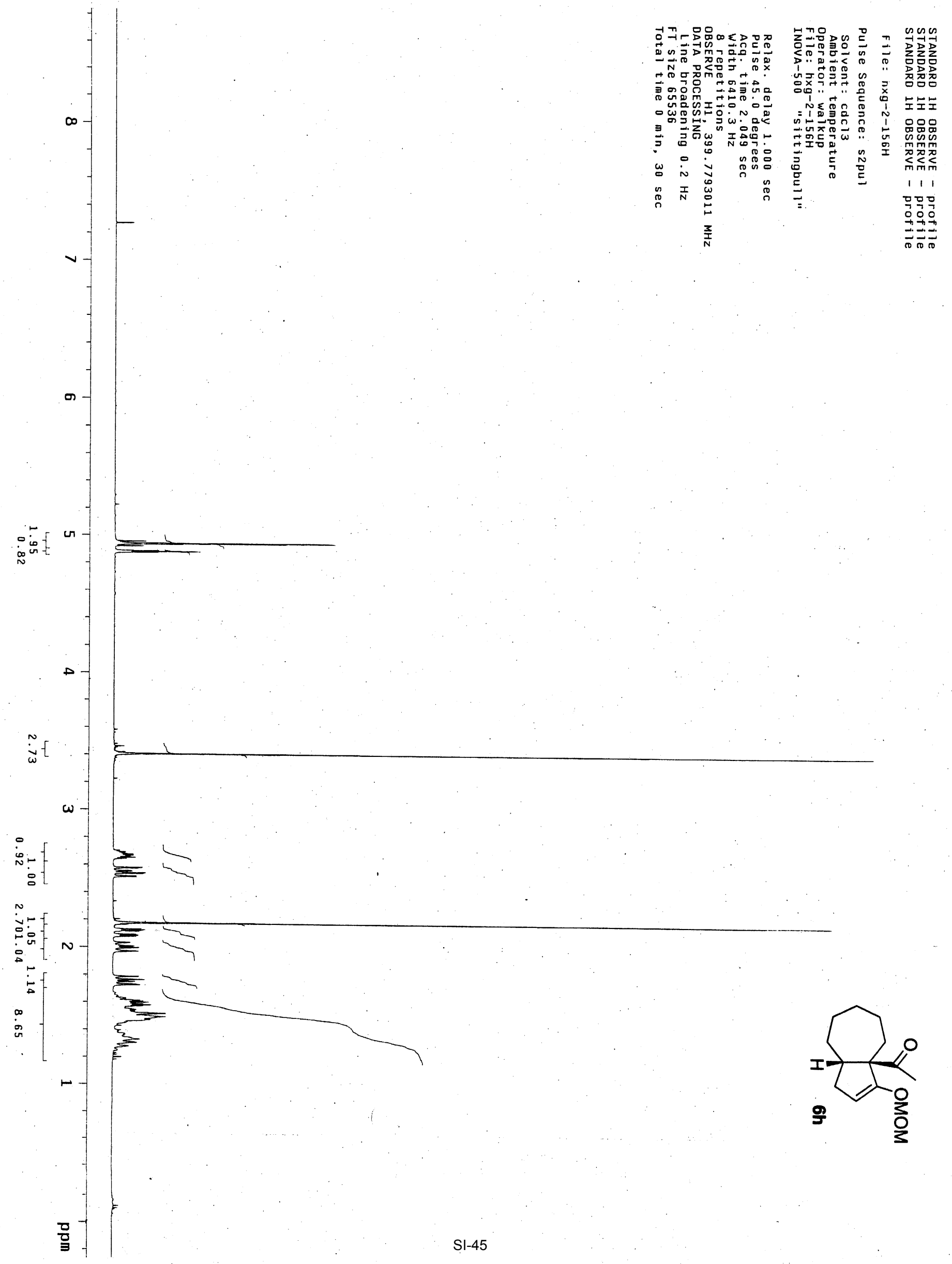




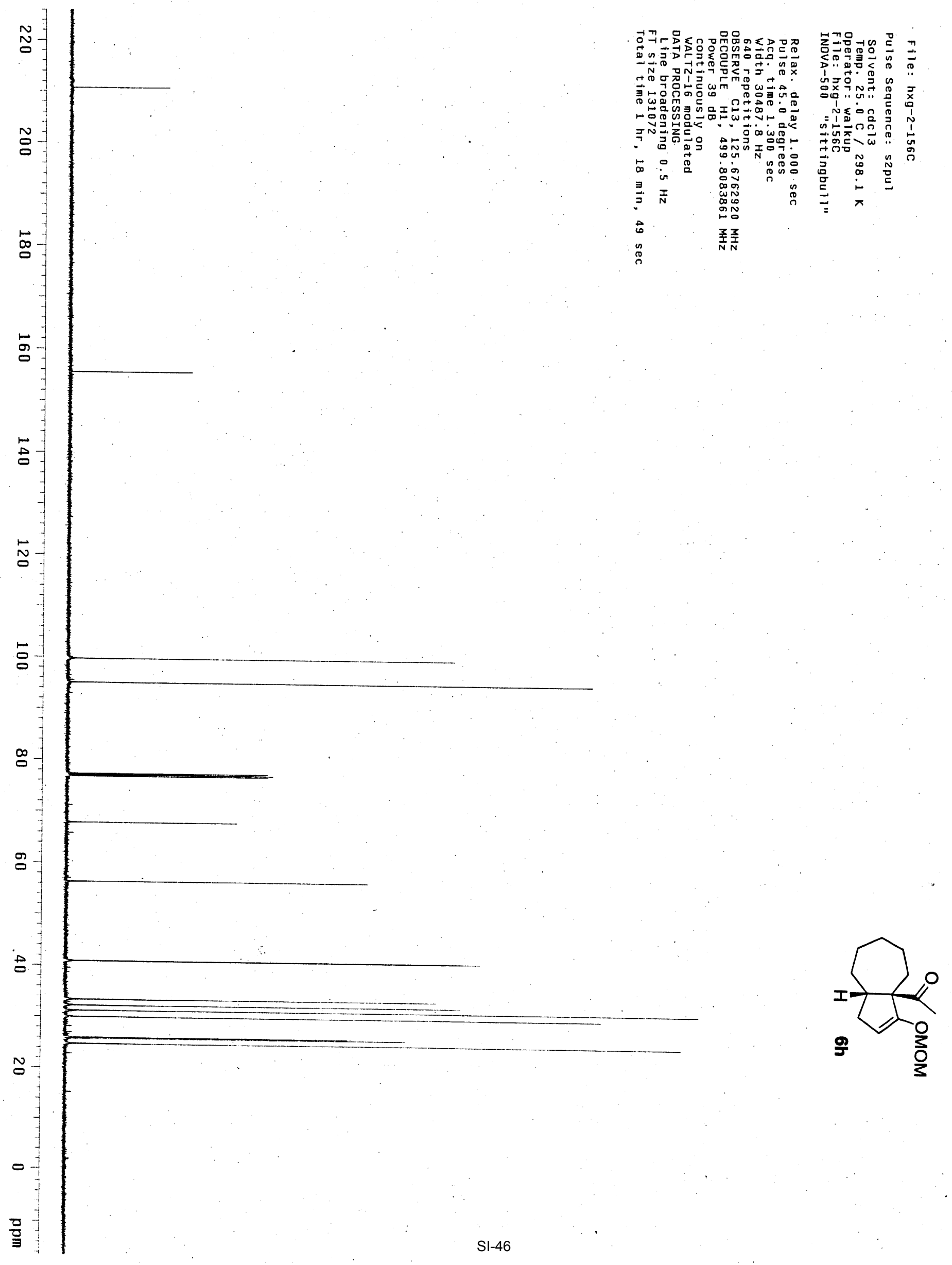




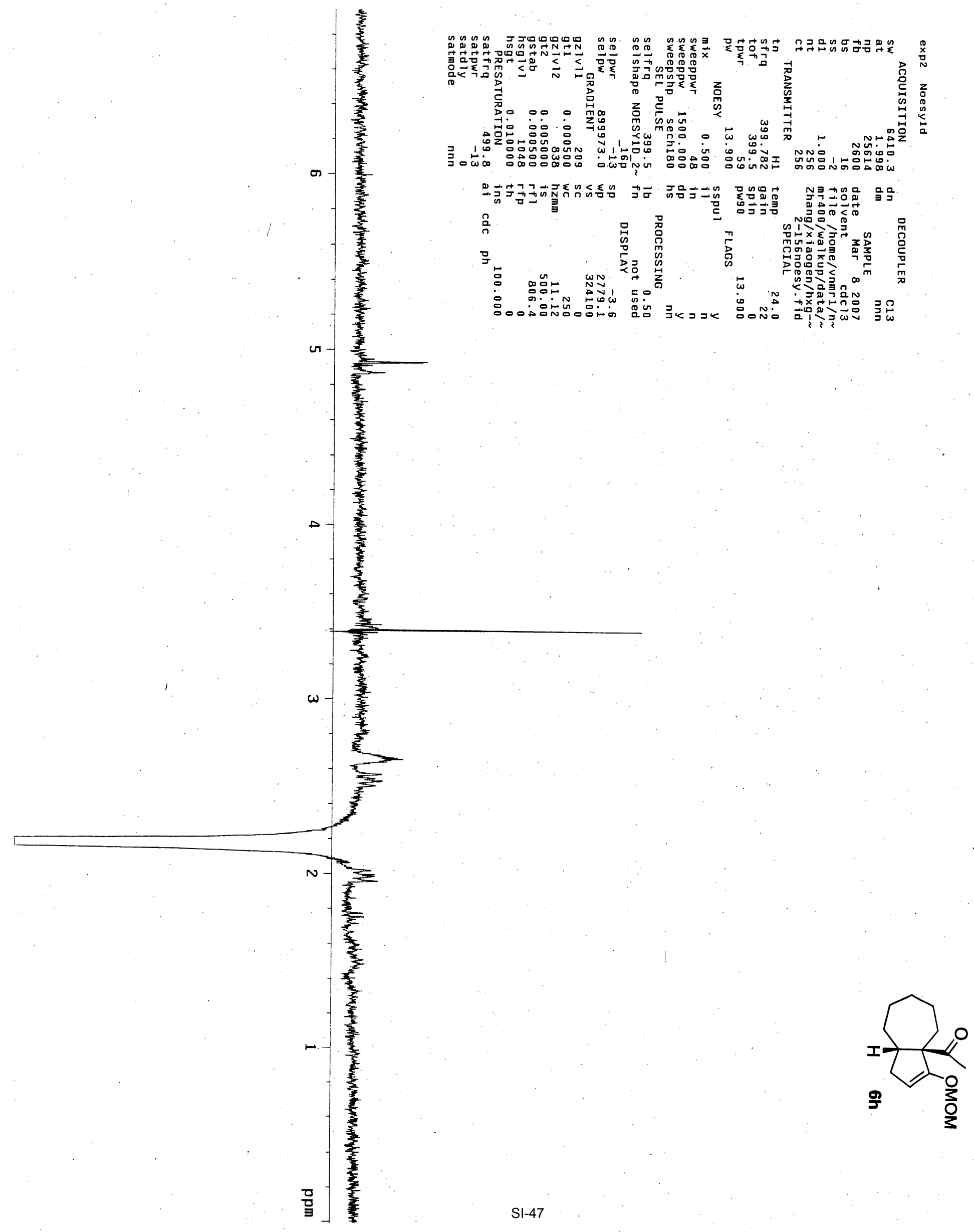




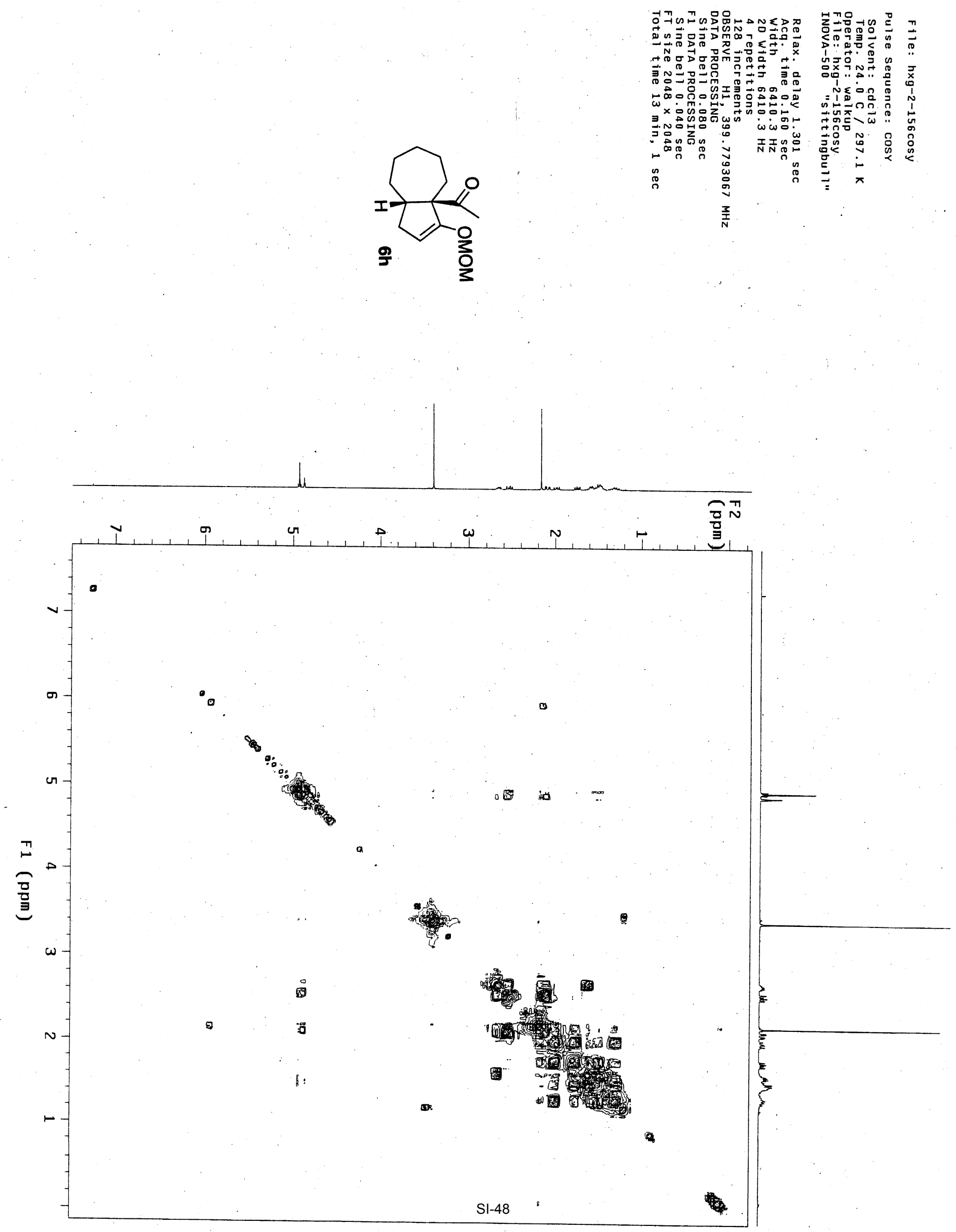



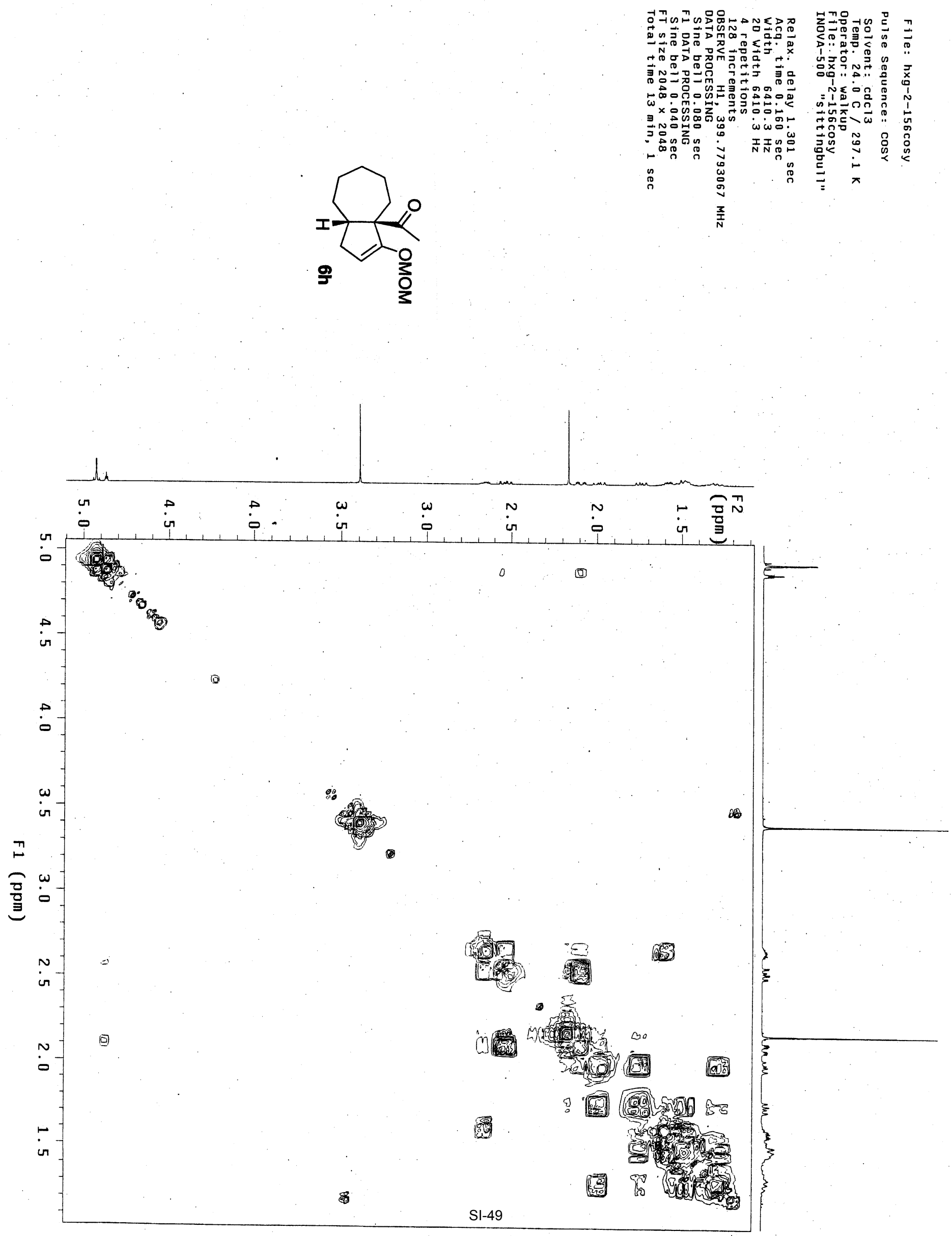


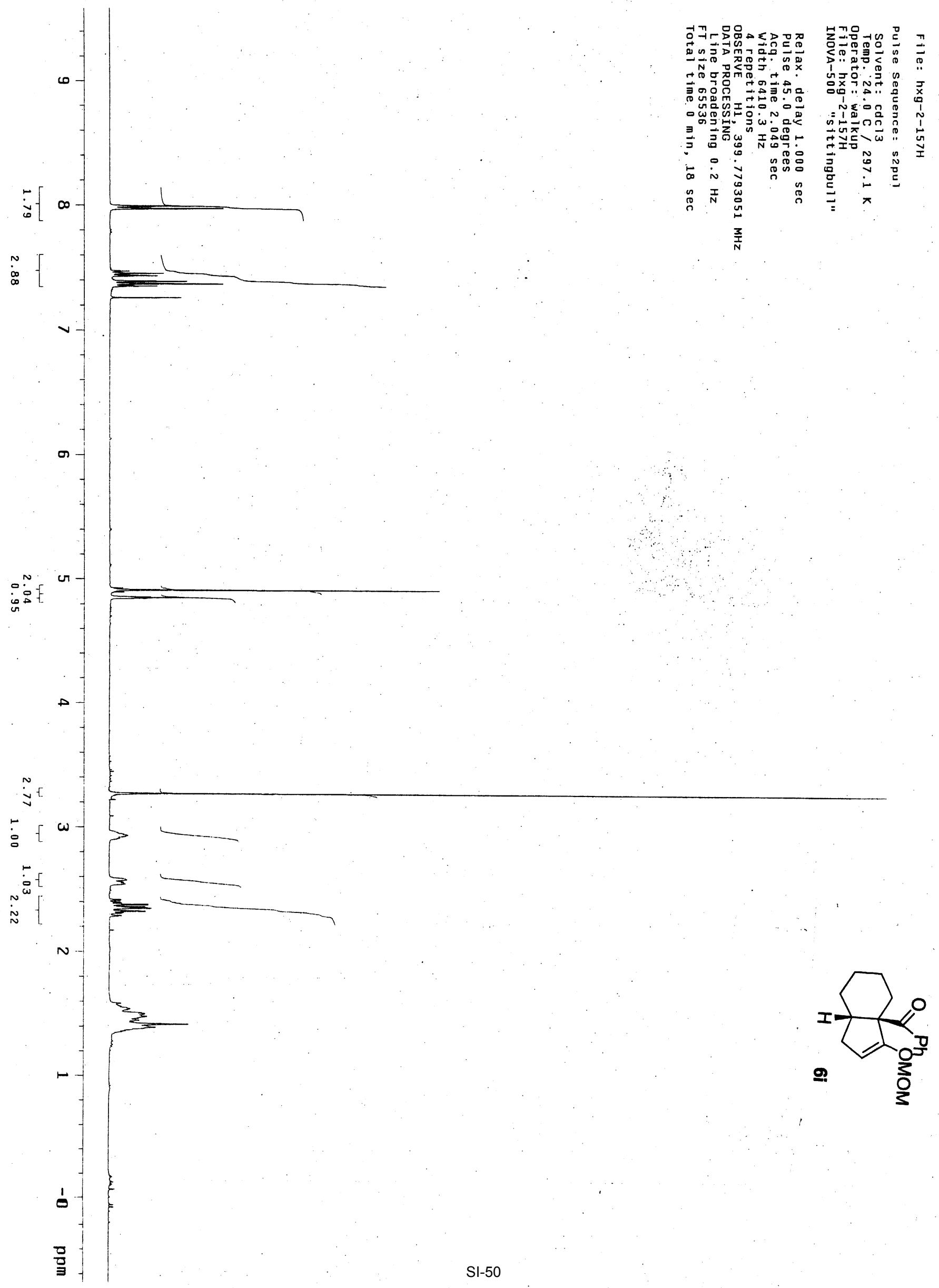




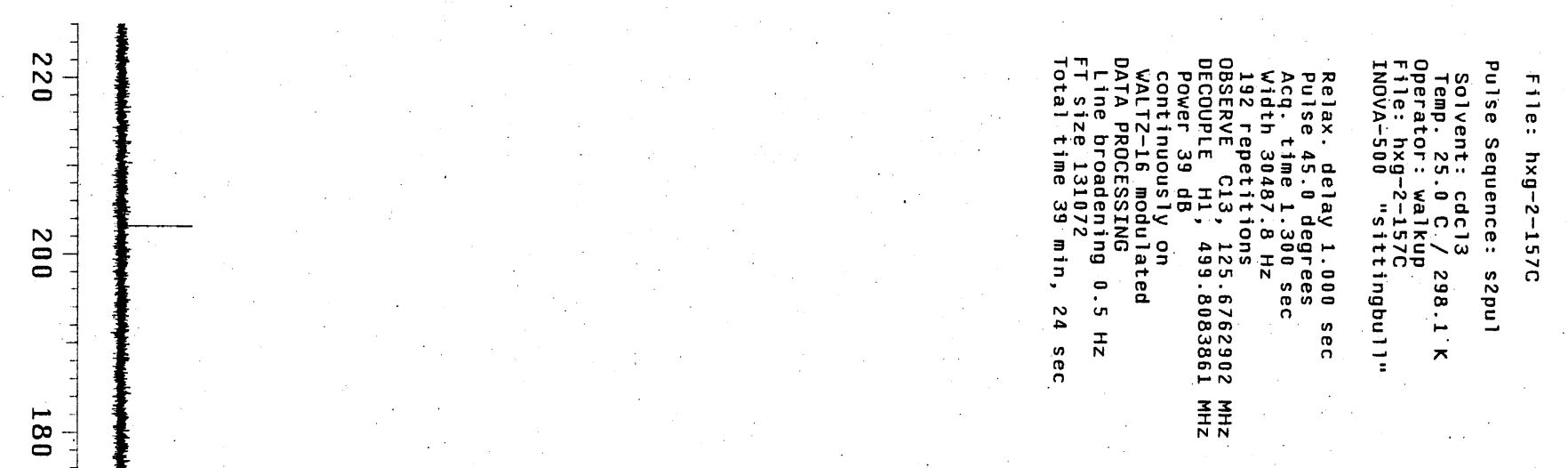

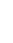

g

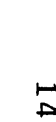

5

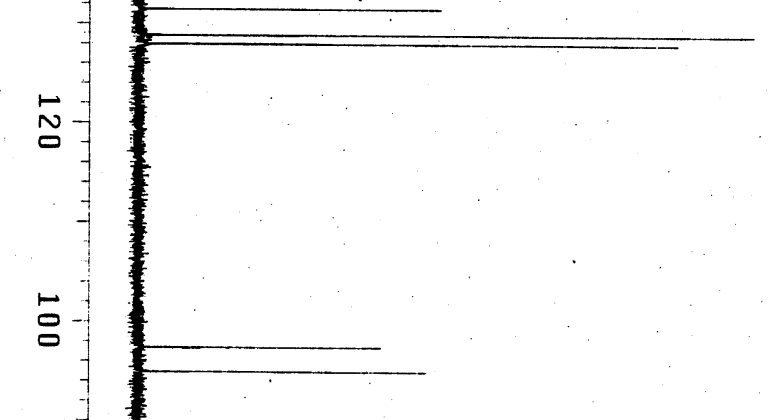

$\stackrel{\infty}{\infty}$

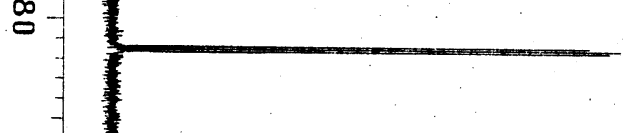

g

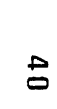

s

$+$
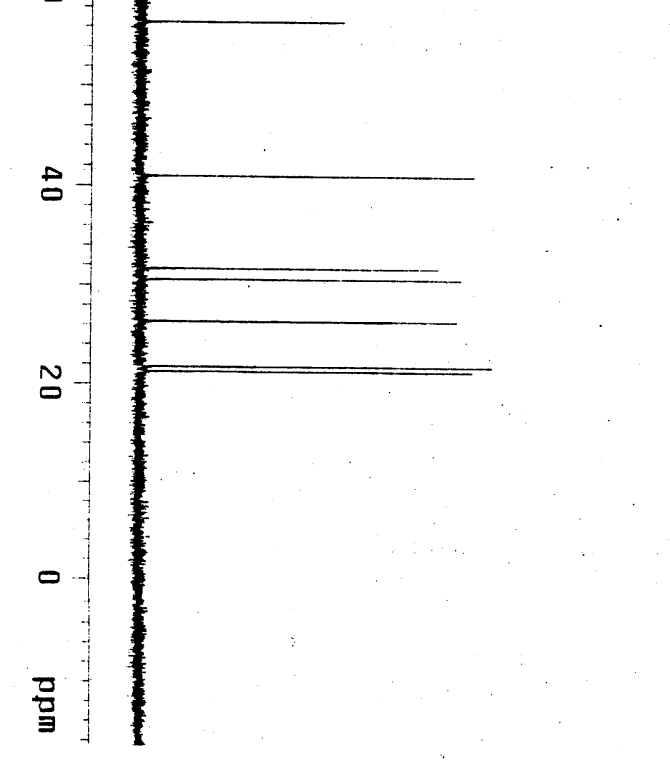


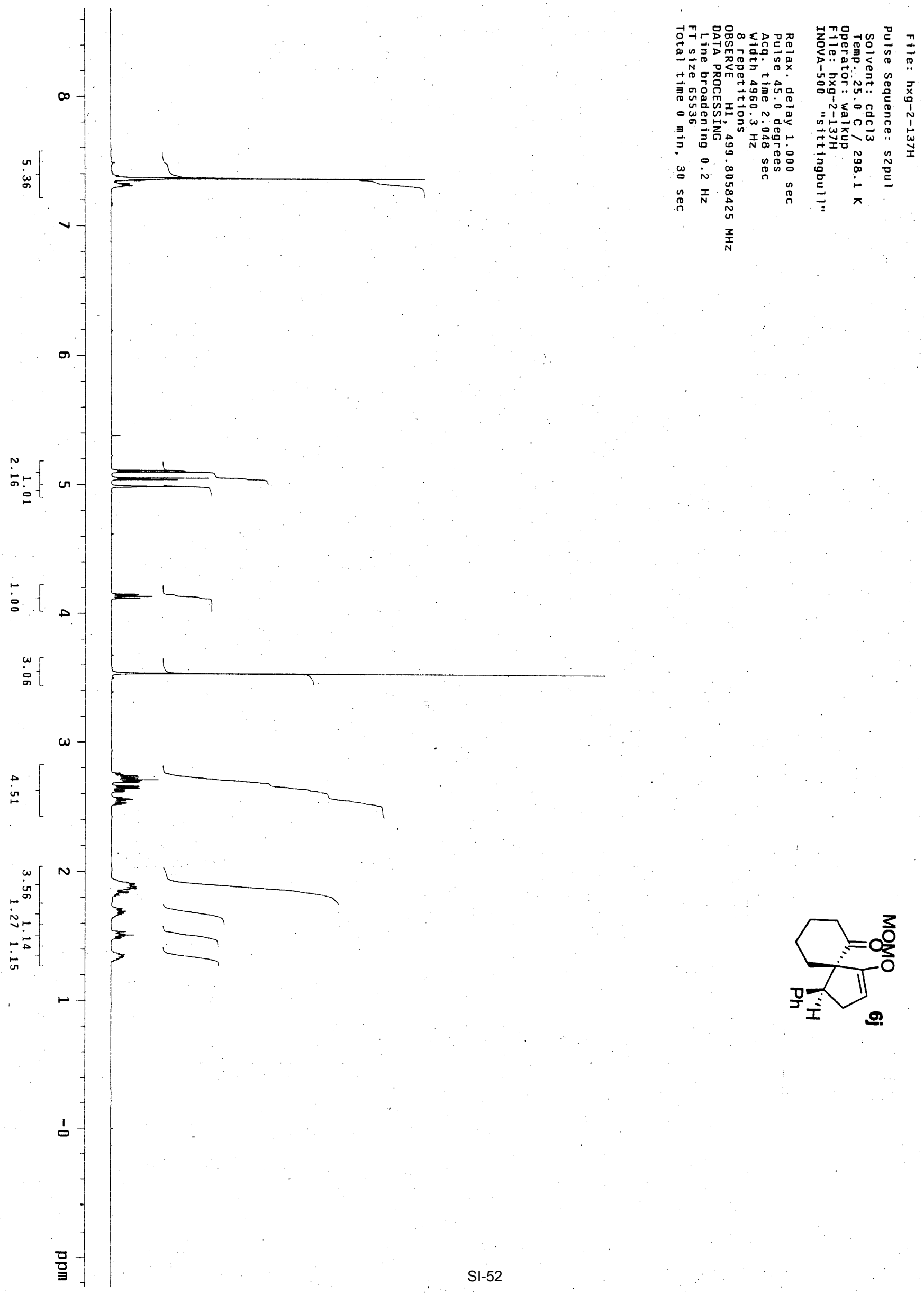




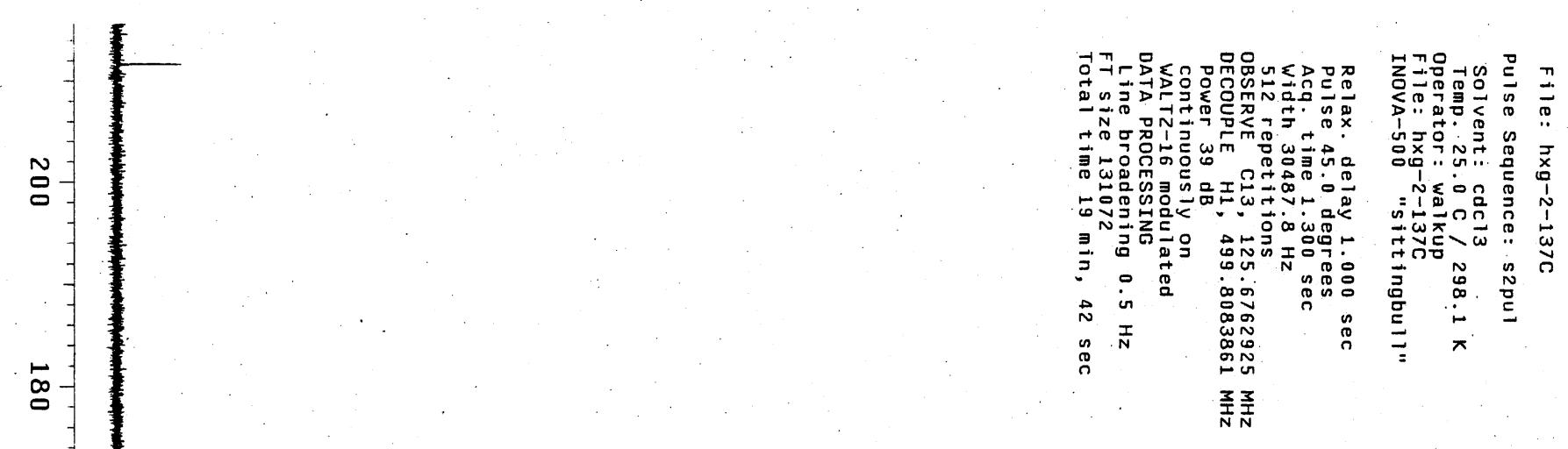

向

$\stackrel{-}{\square}$

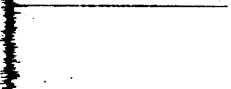

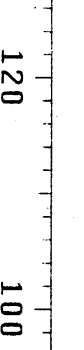

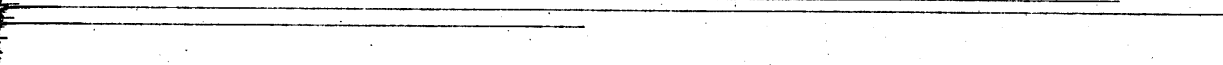

$\stackrel{\infty}{\circ}$

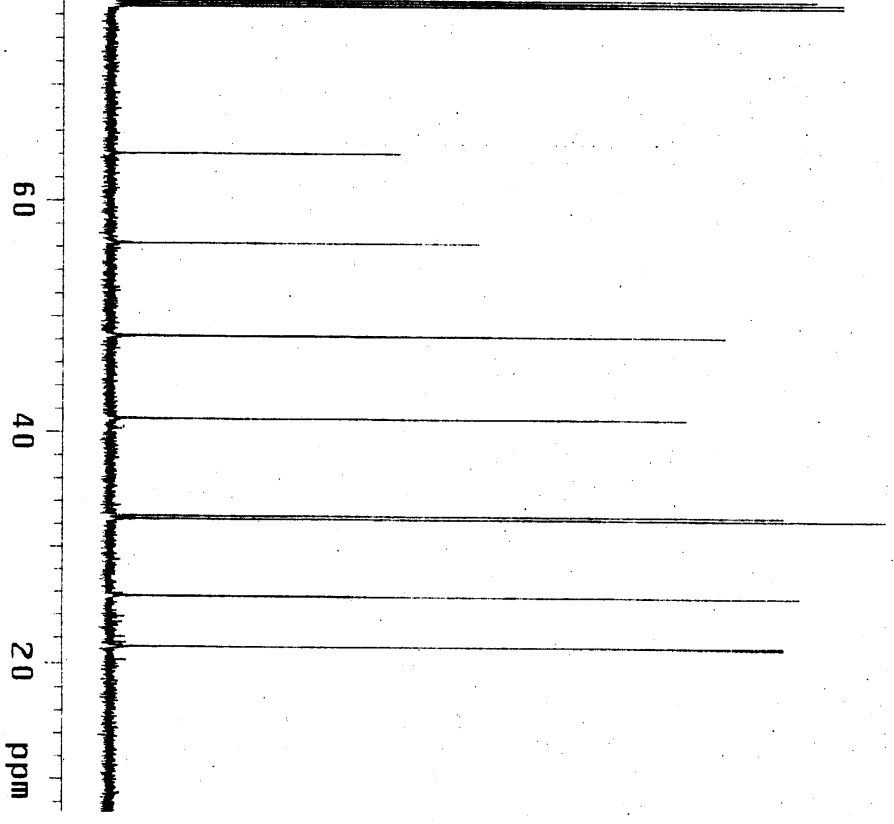




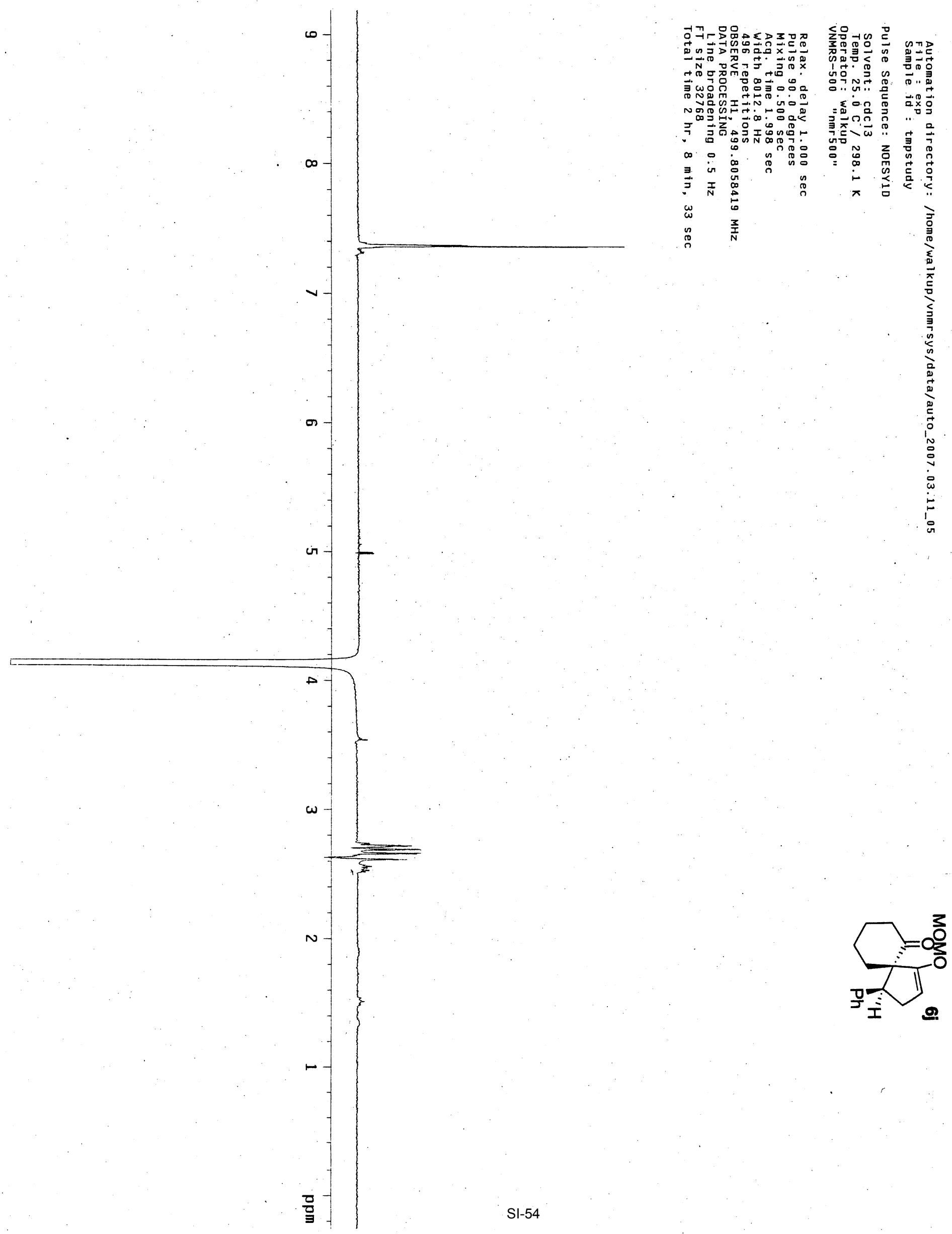




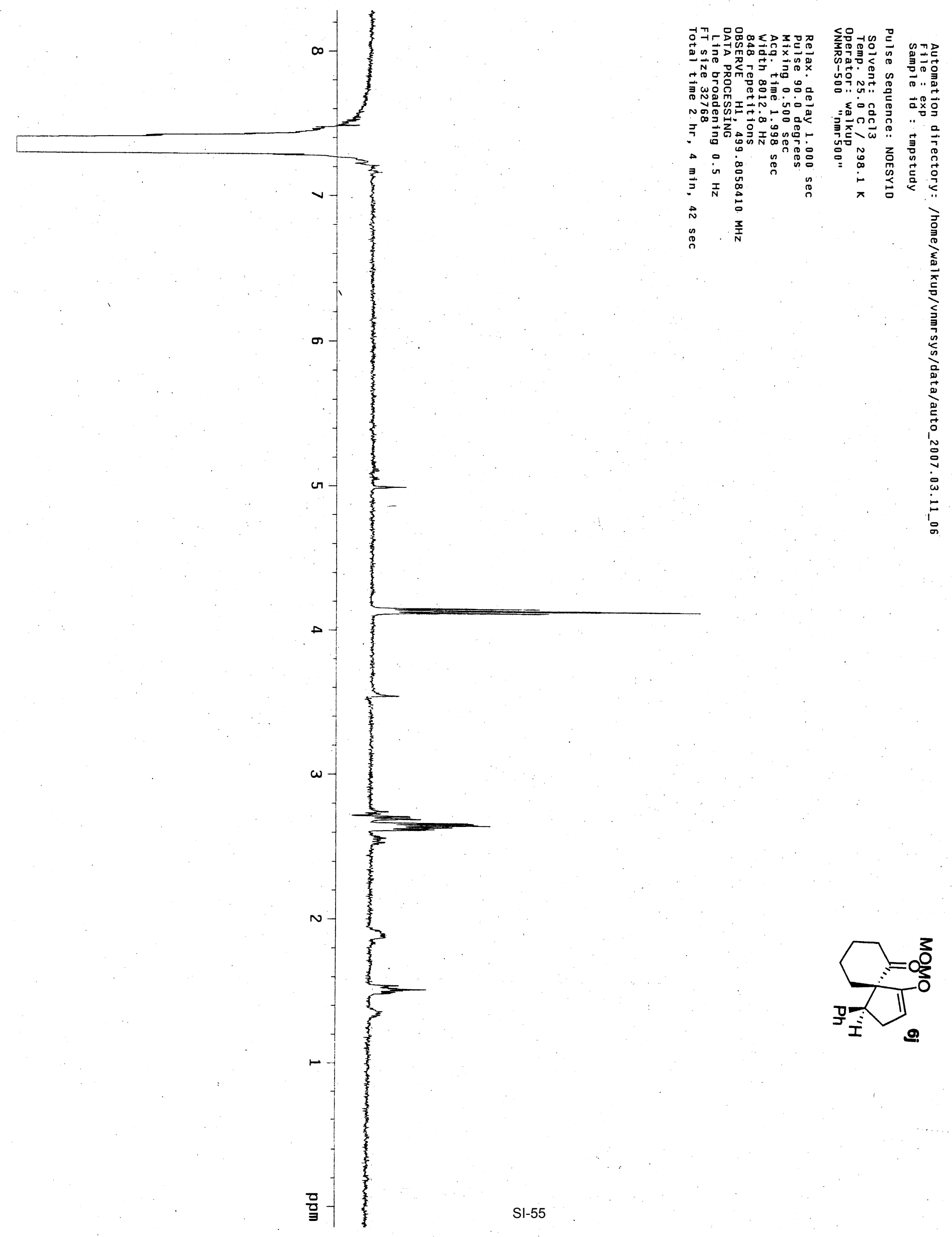



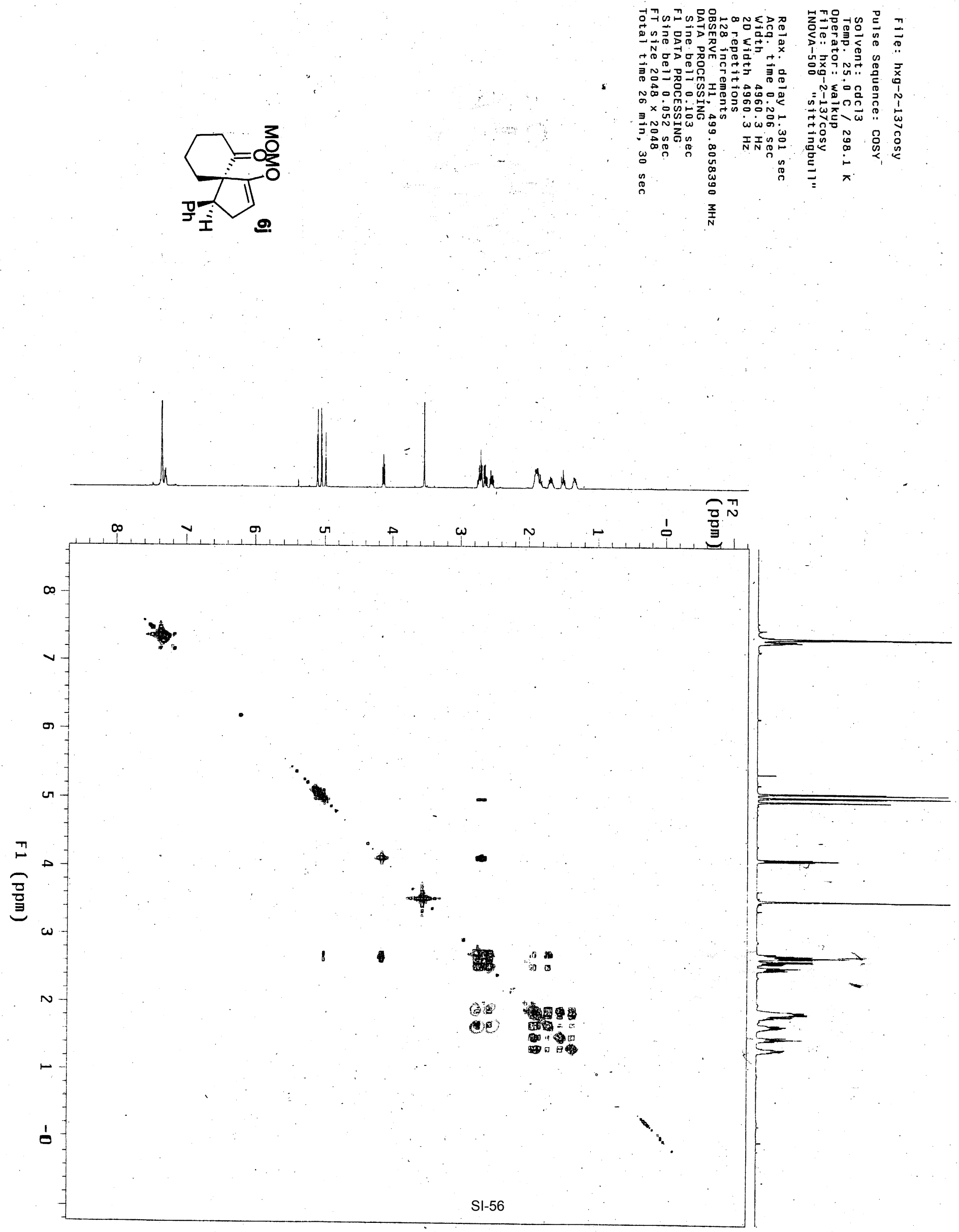

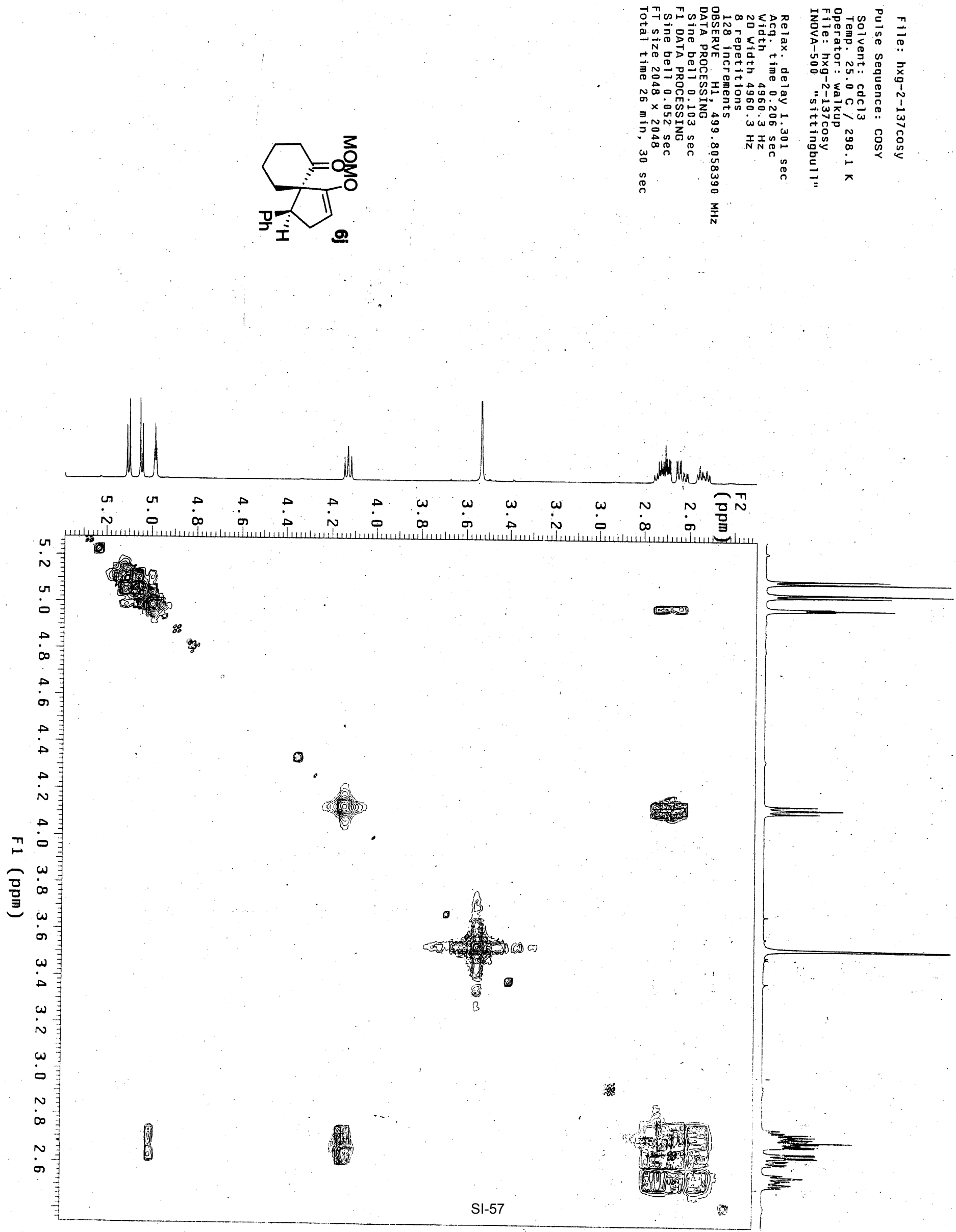


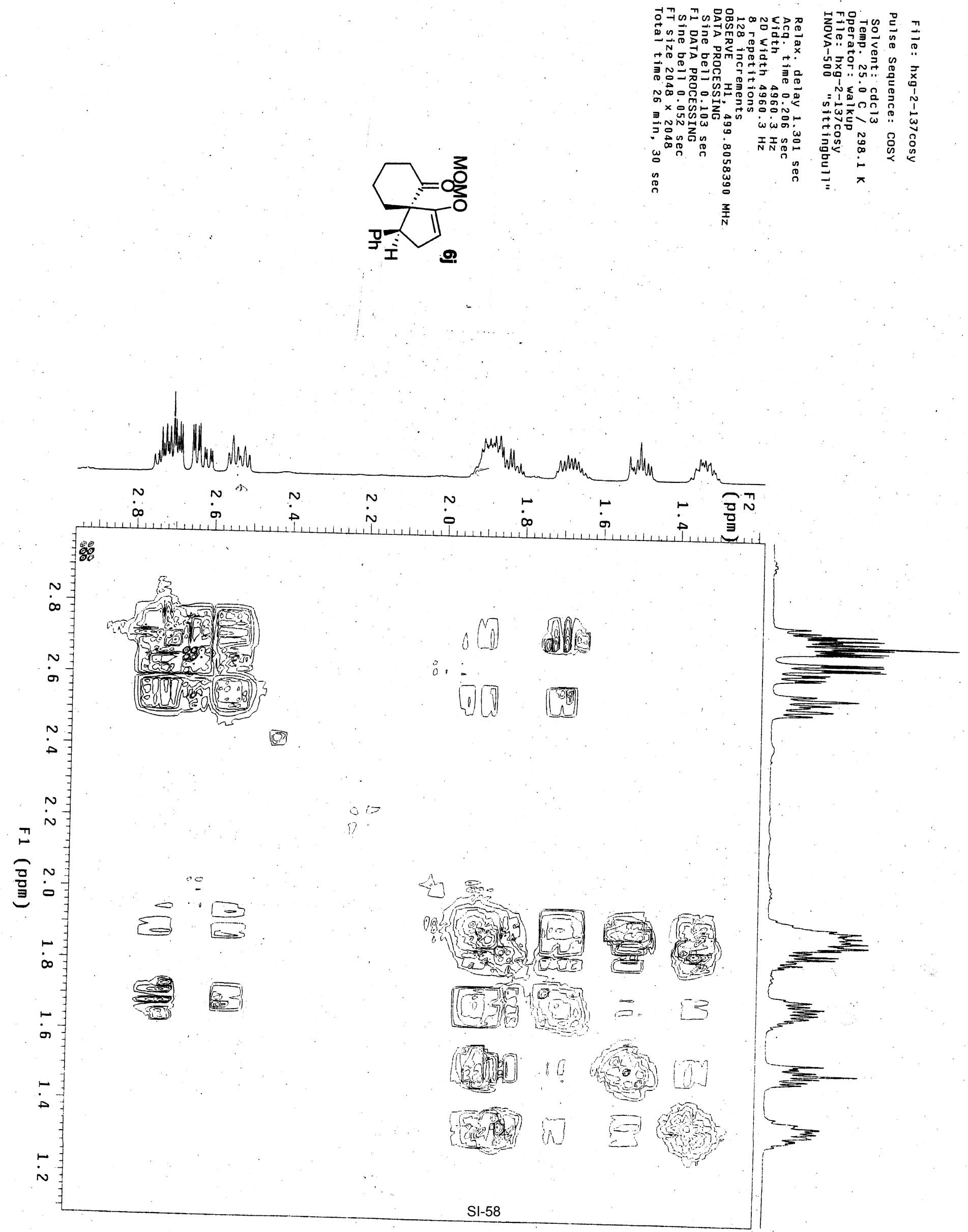




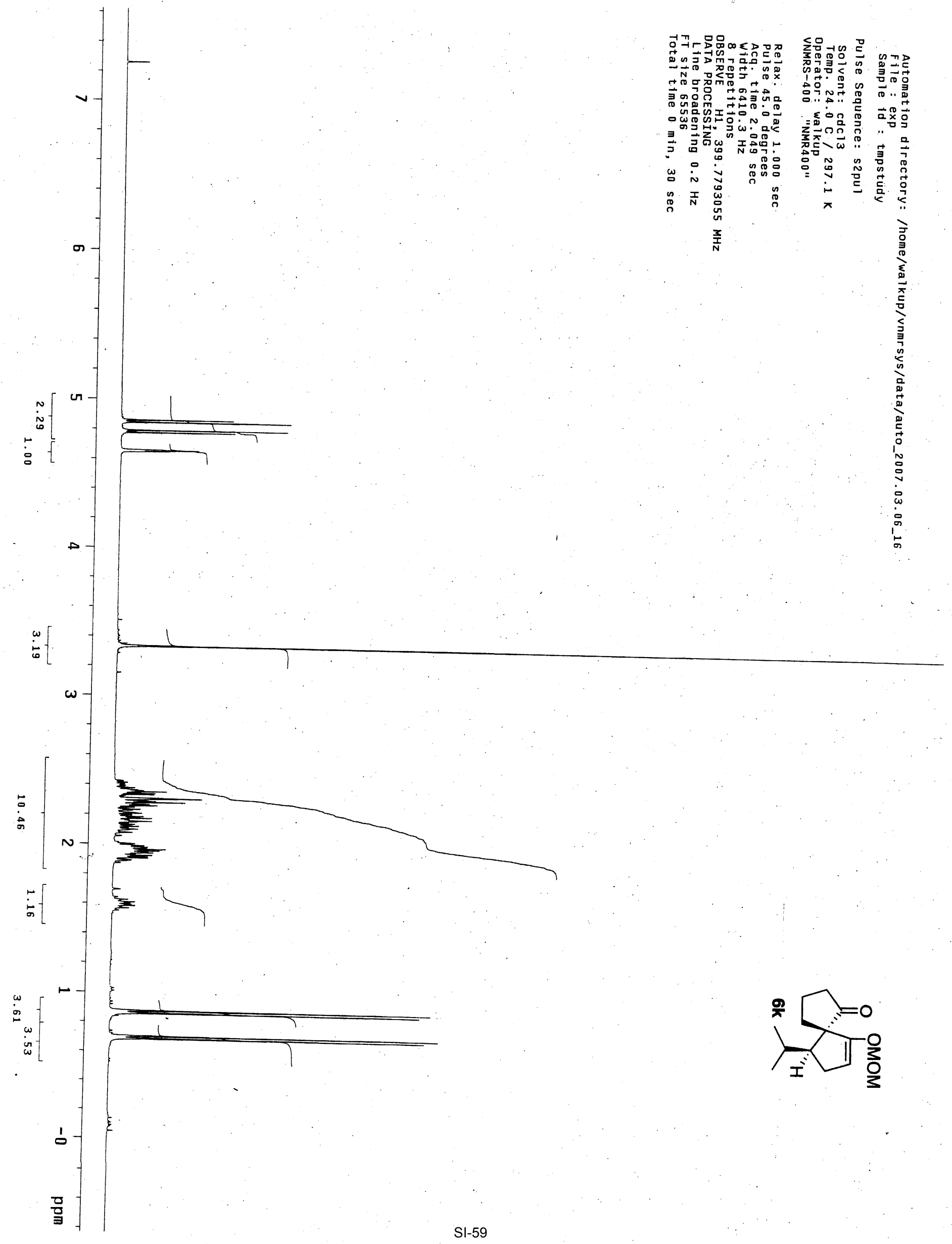




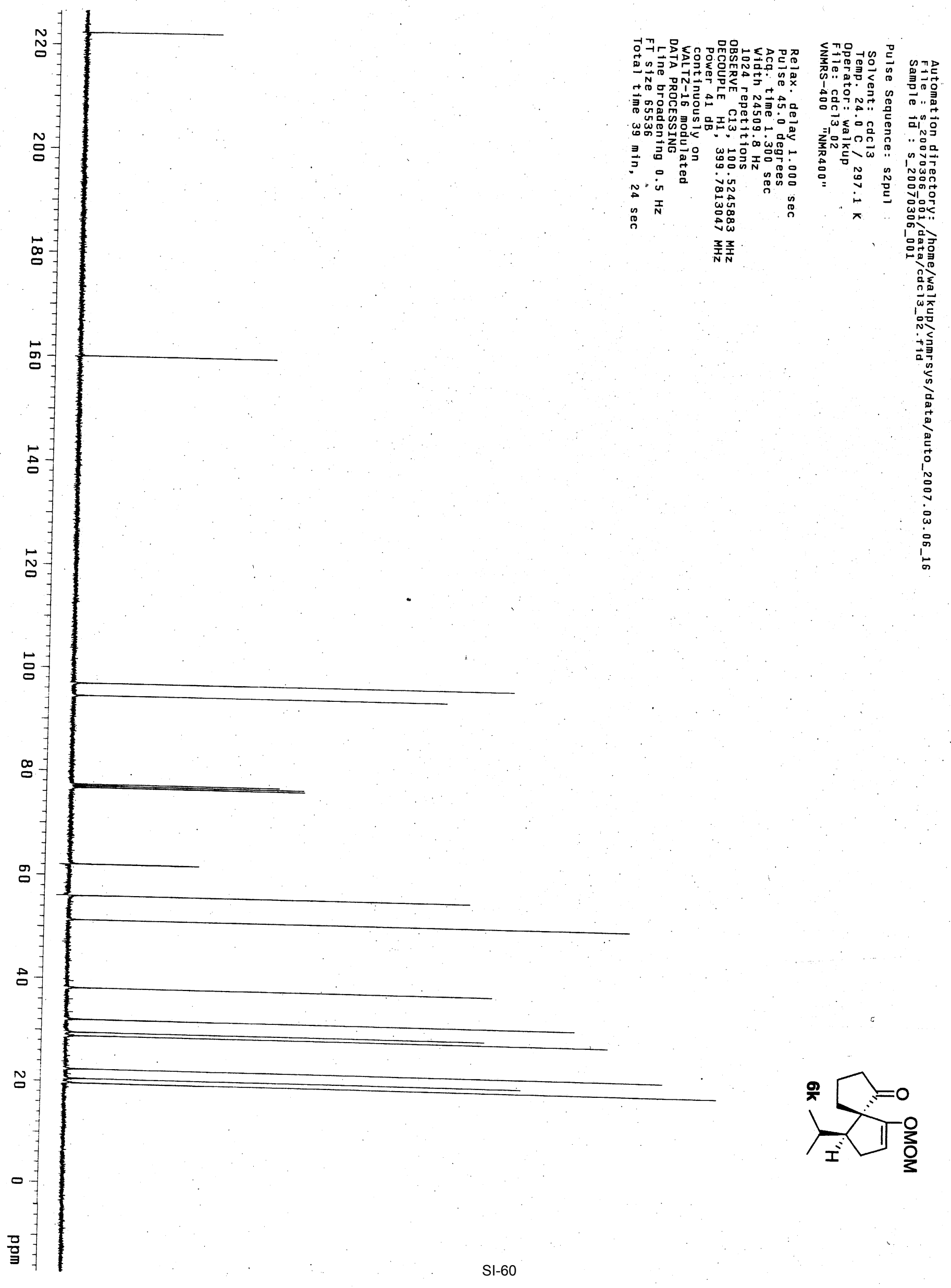




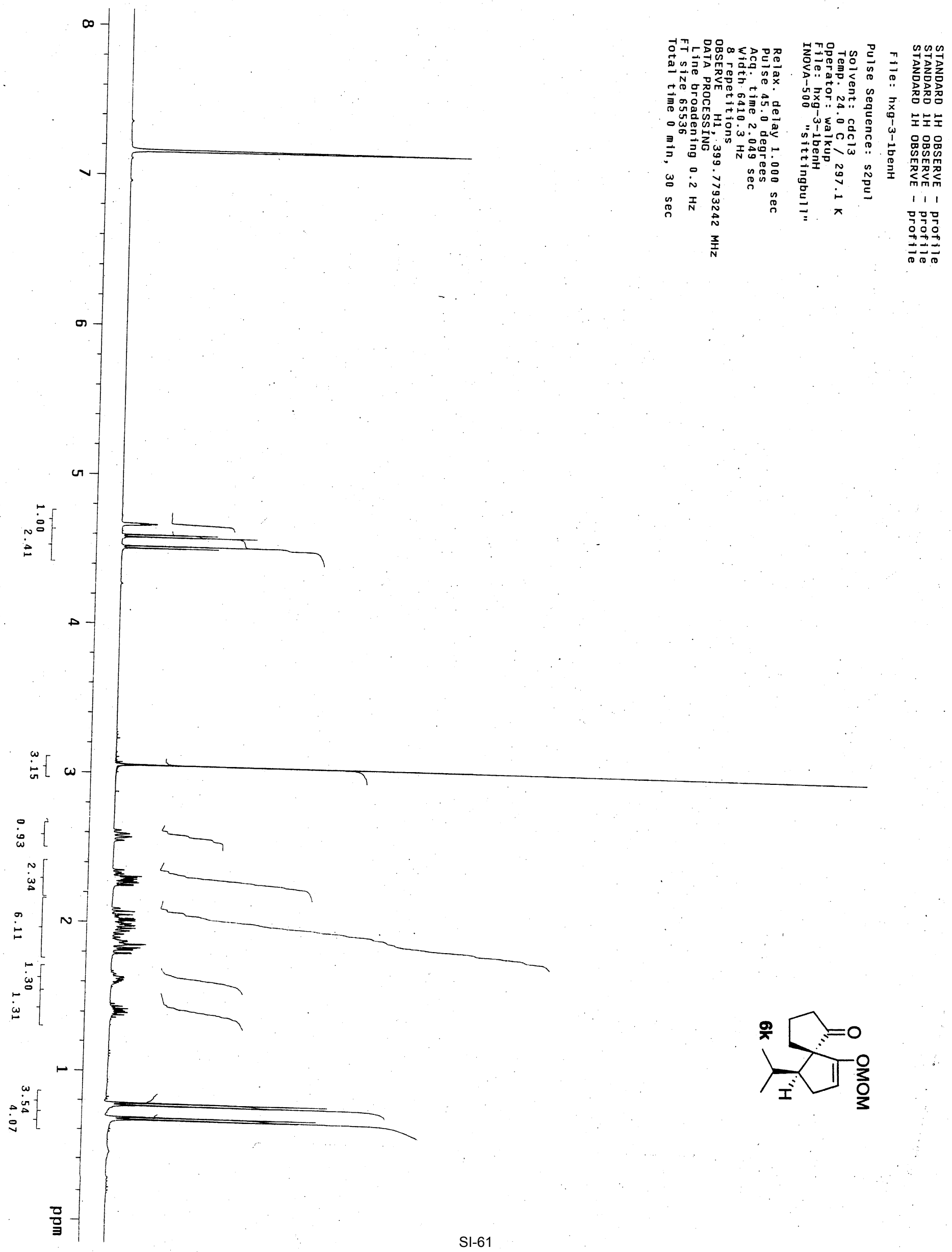




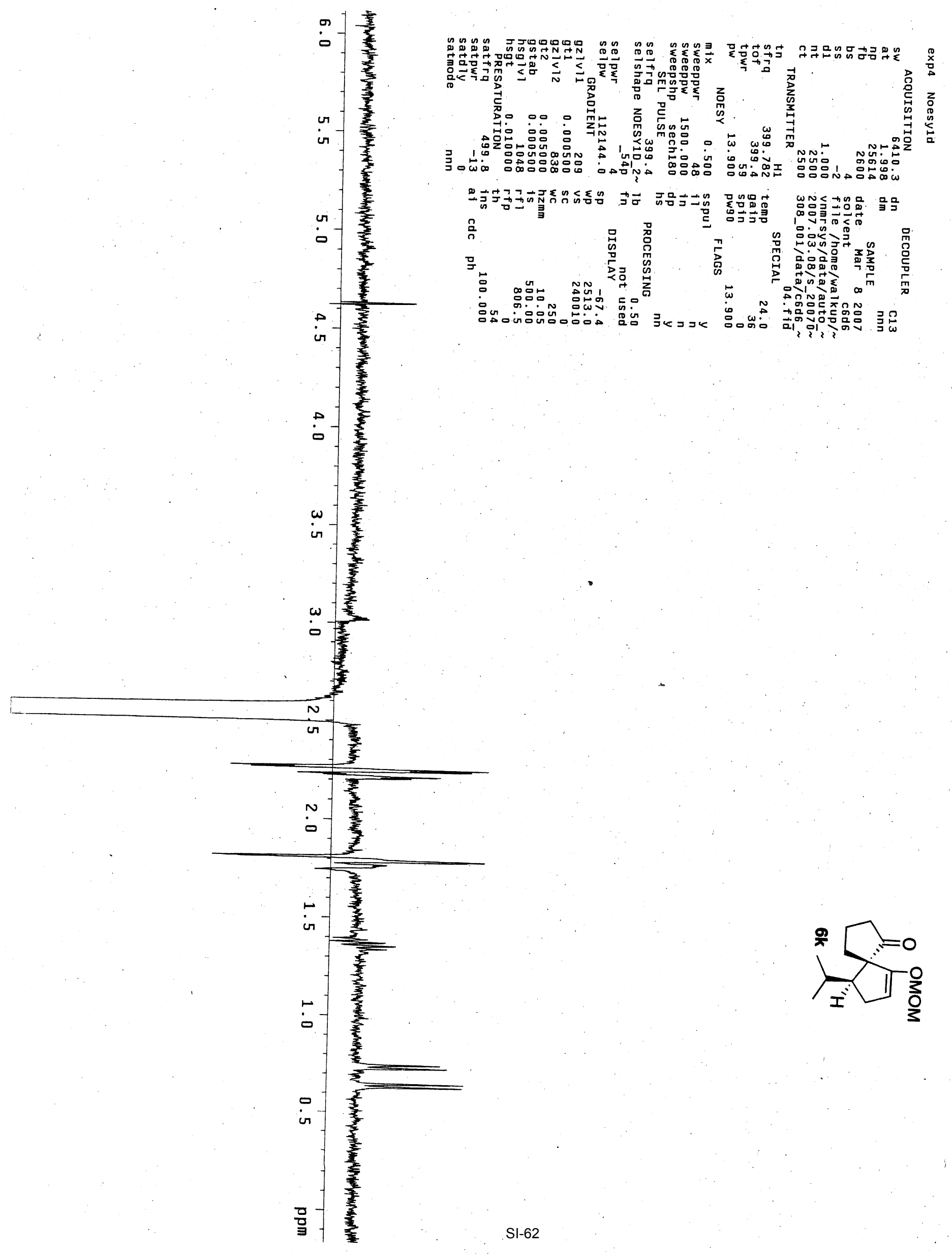



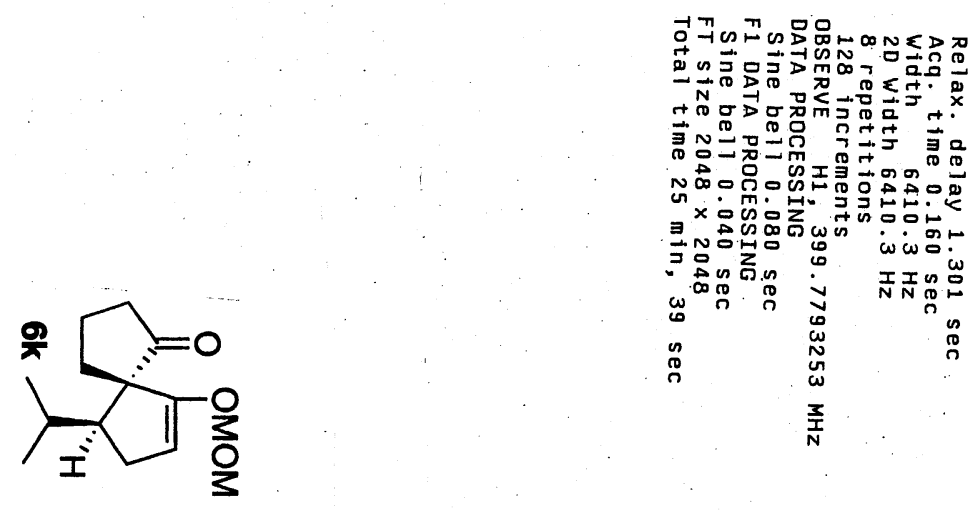

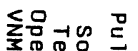

的

年

..P.

주의

袀

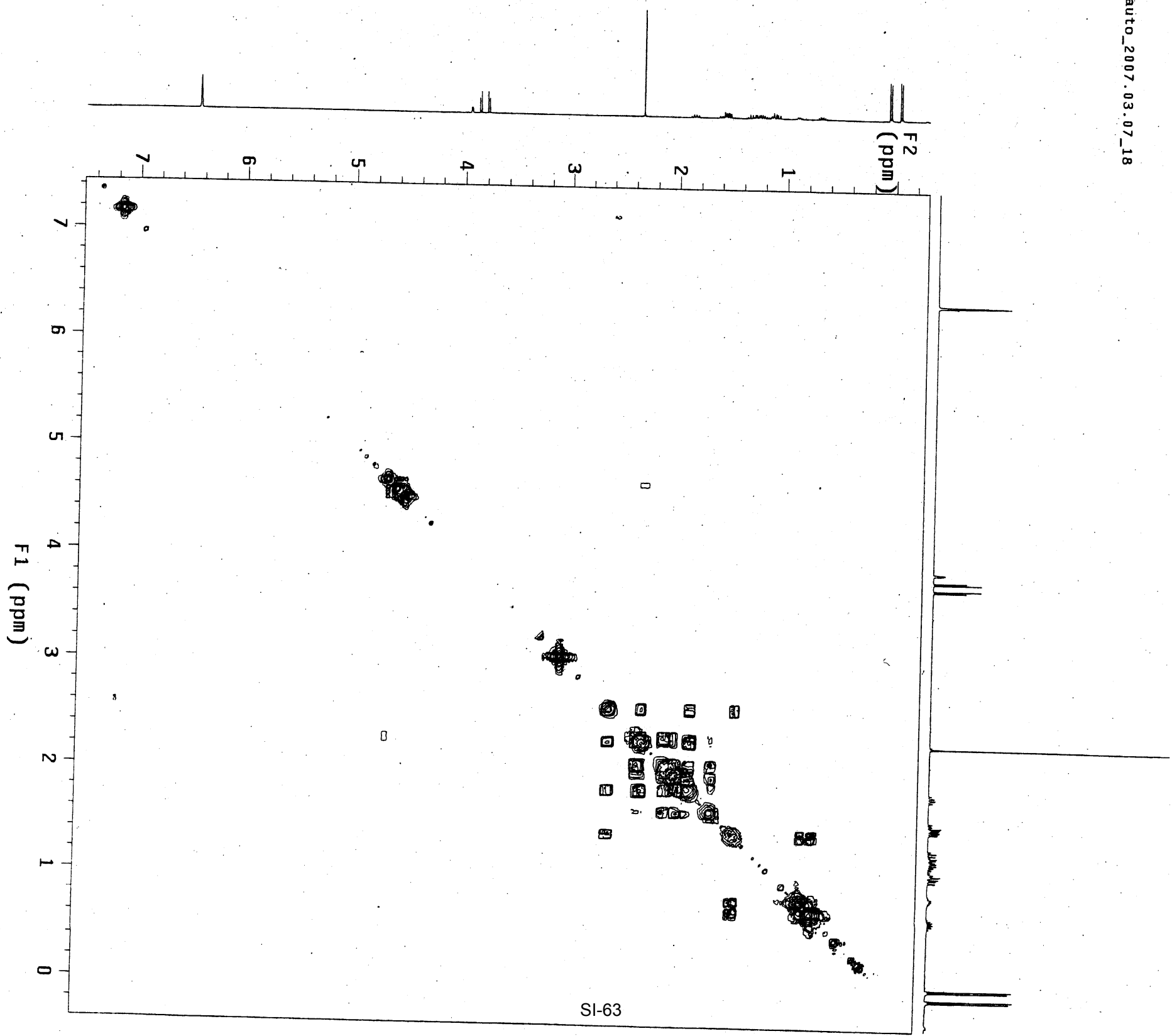




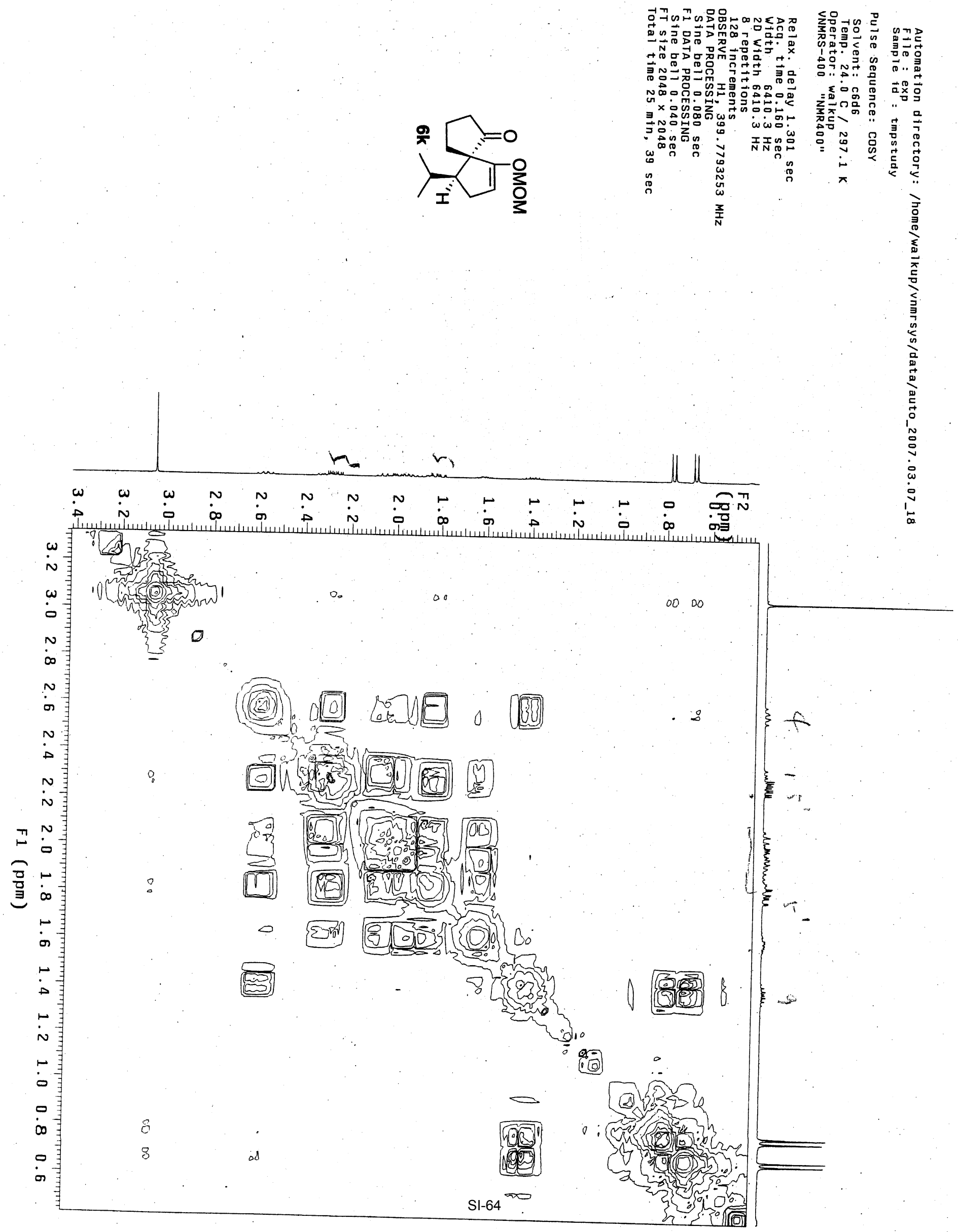




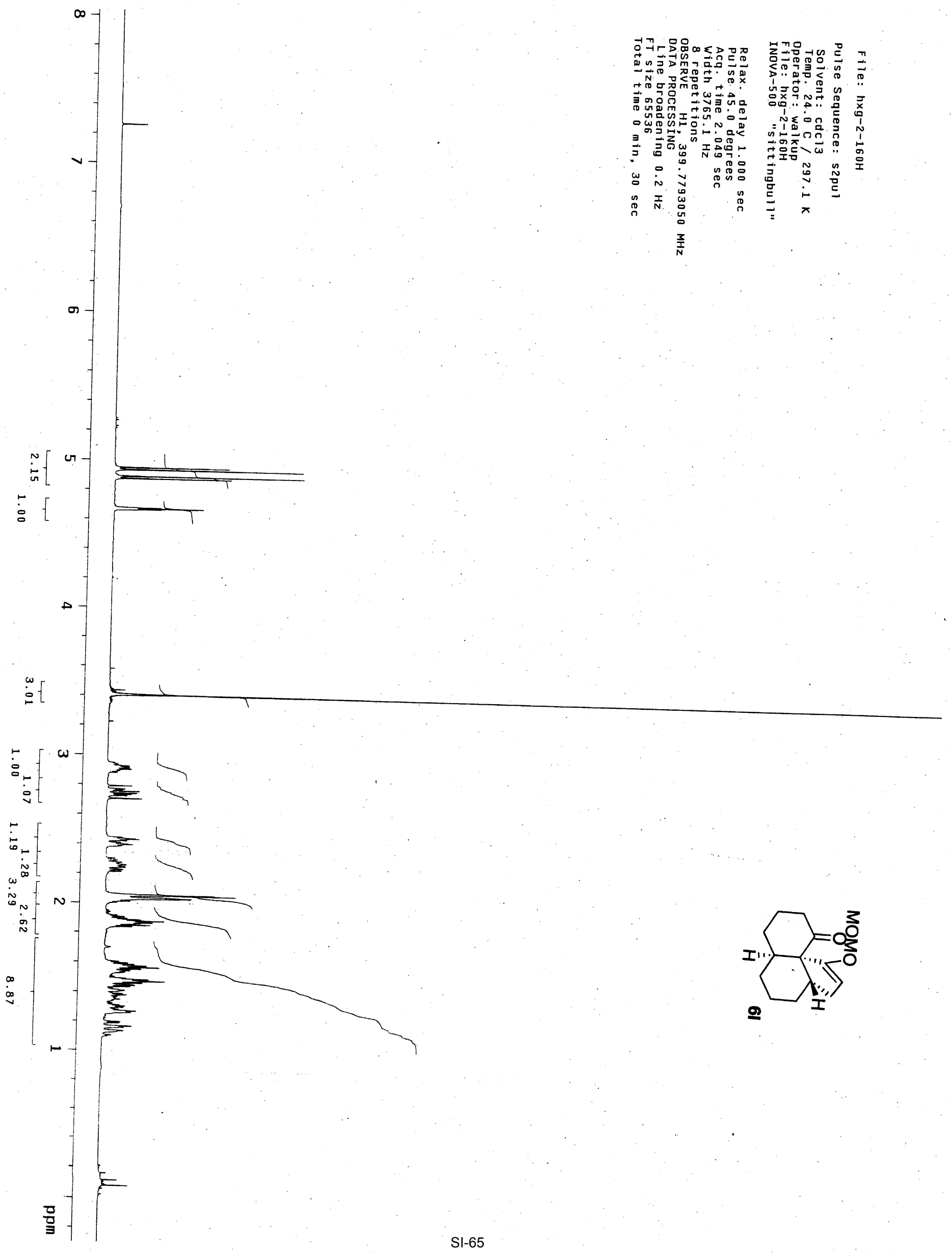



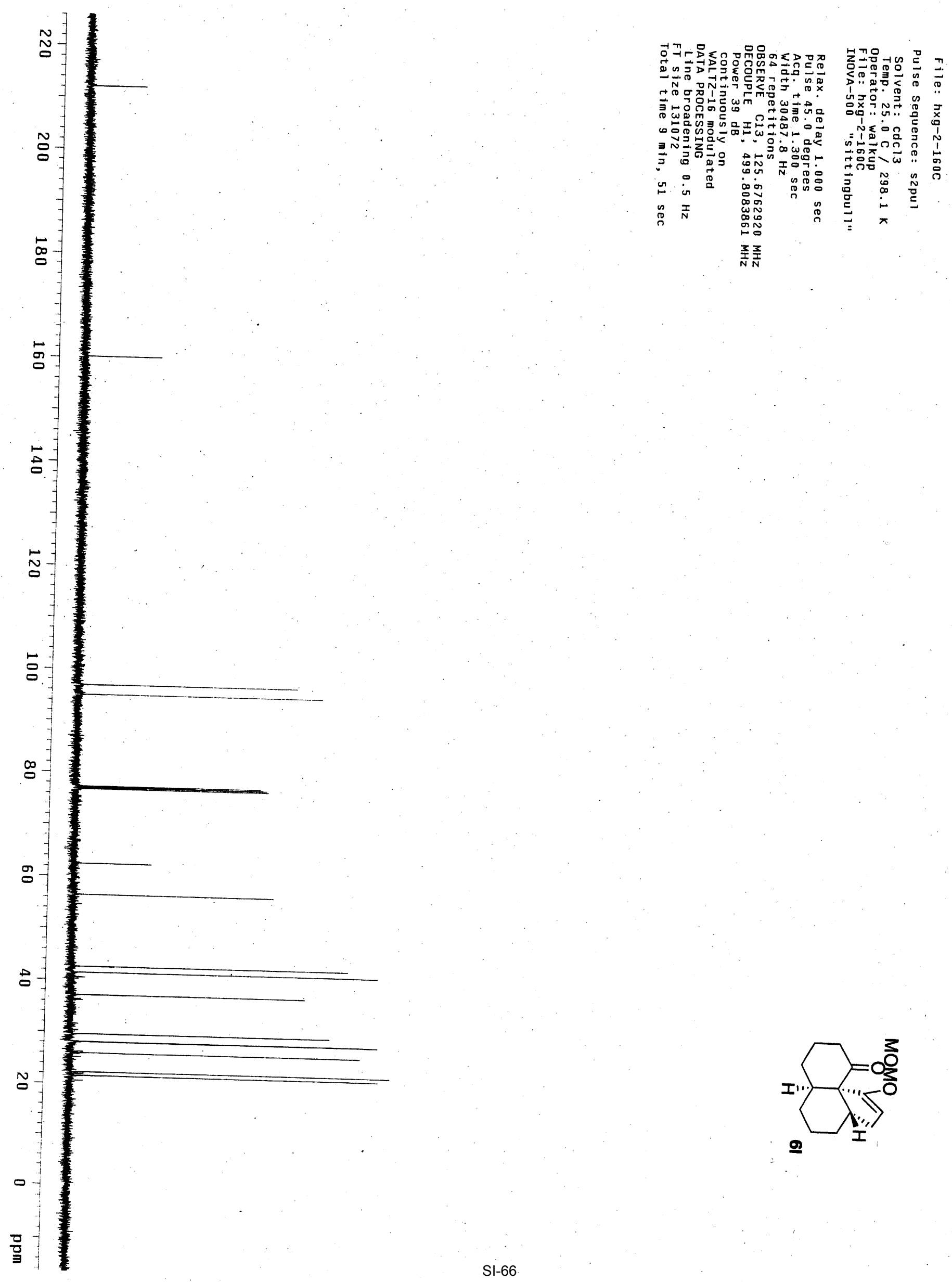


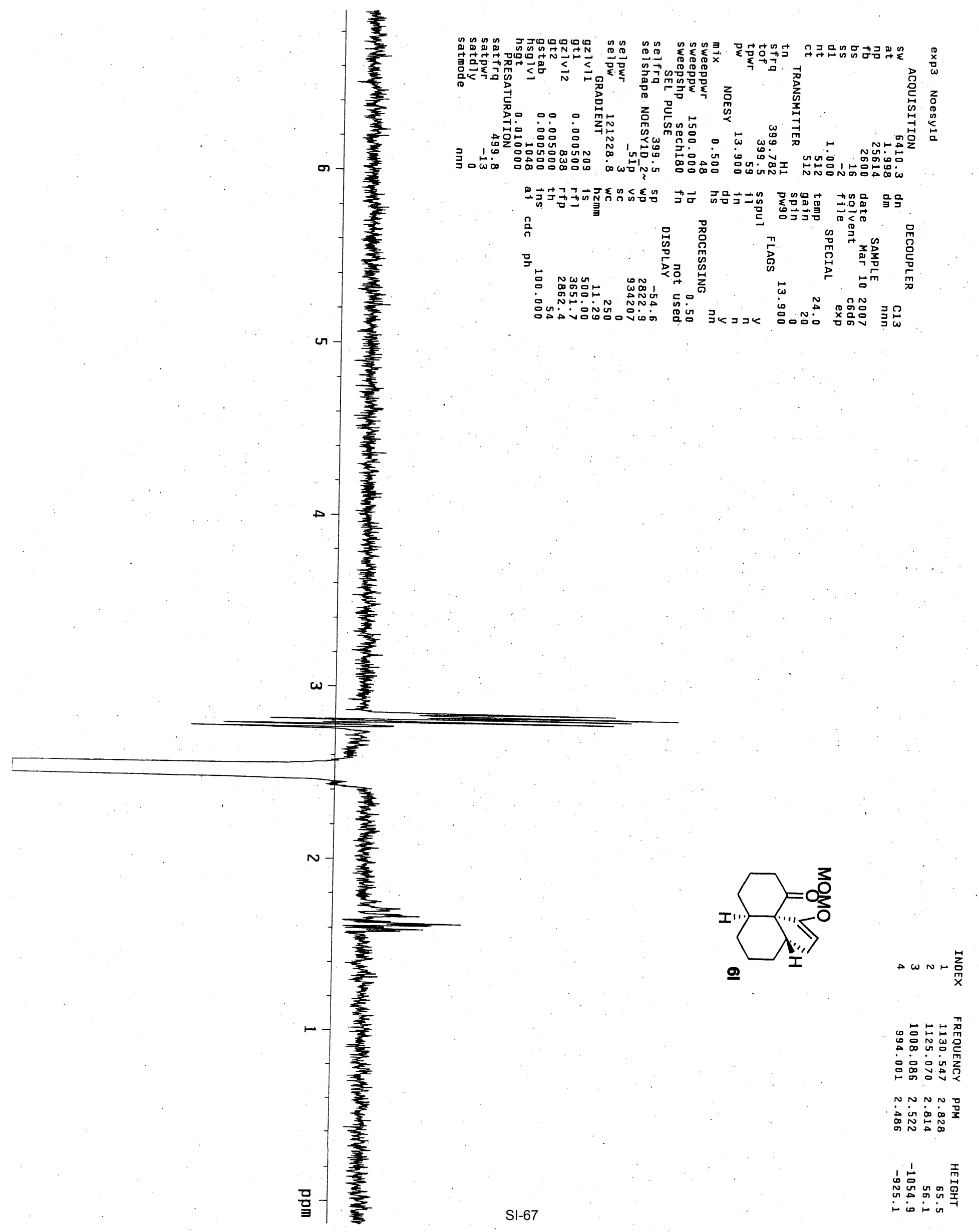




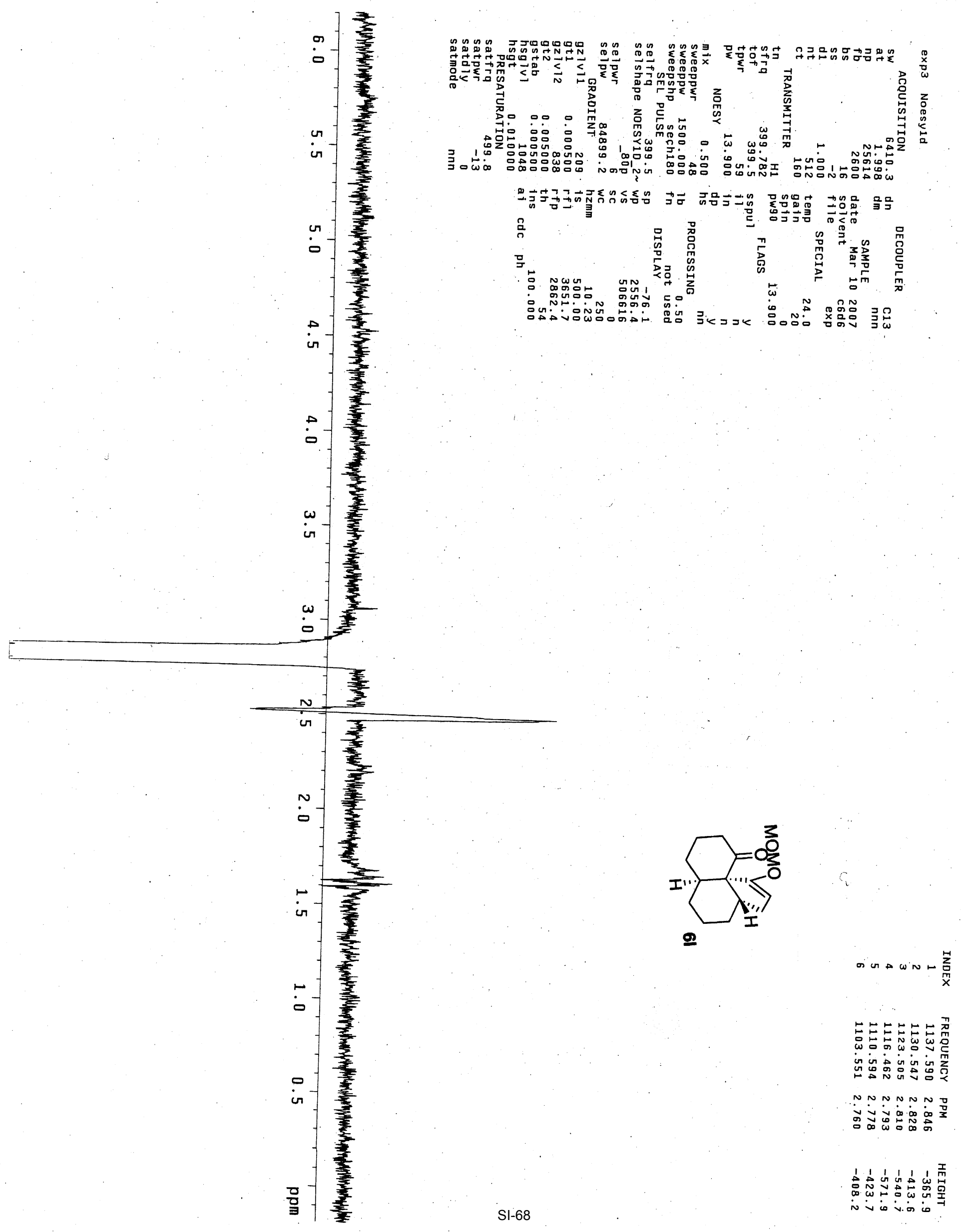




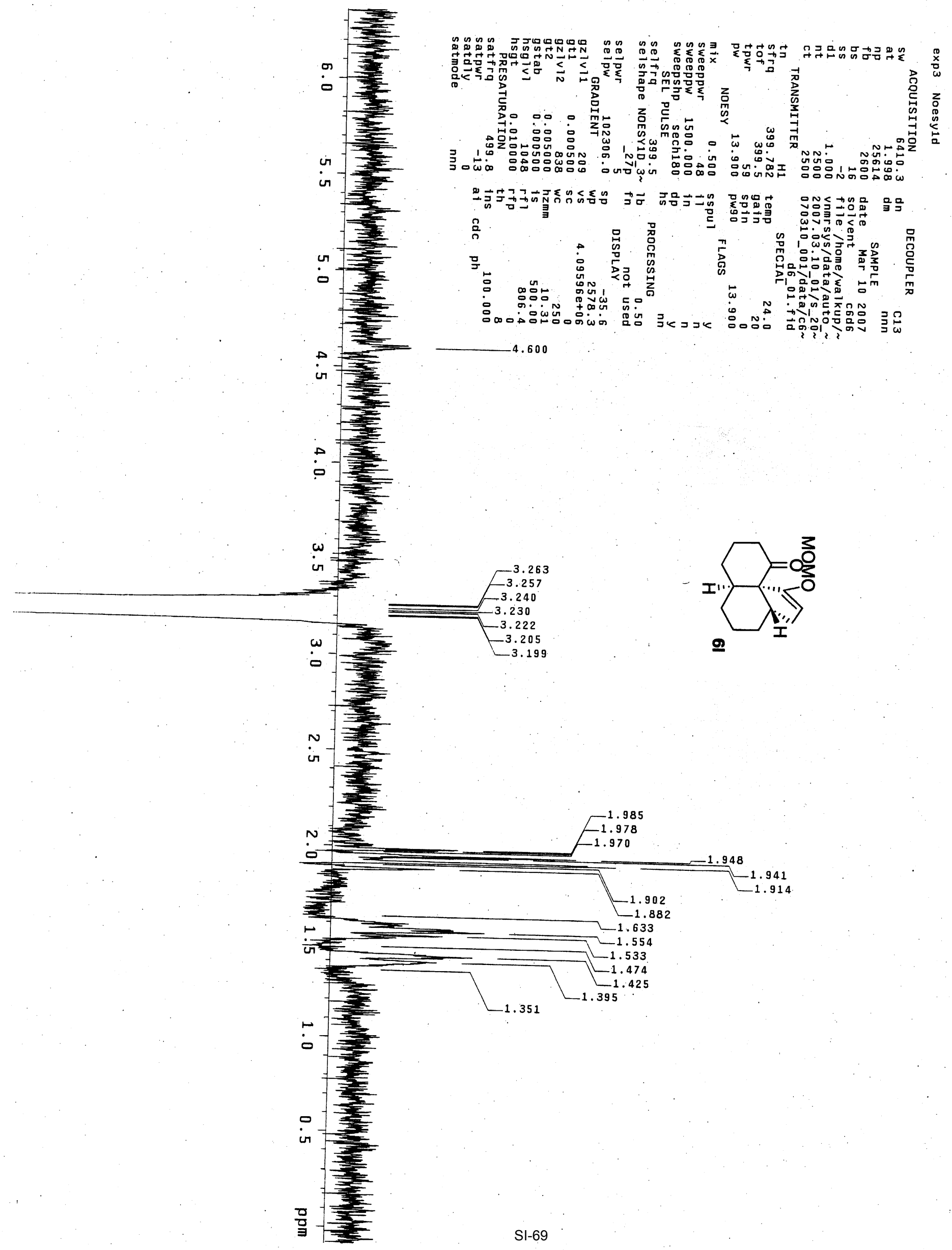




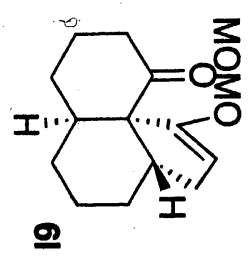
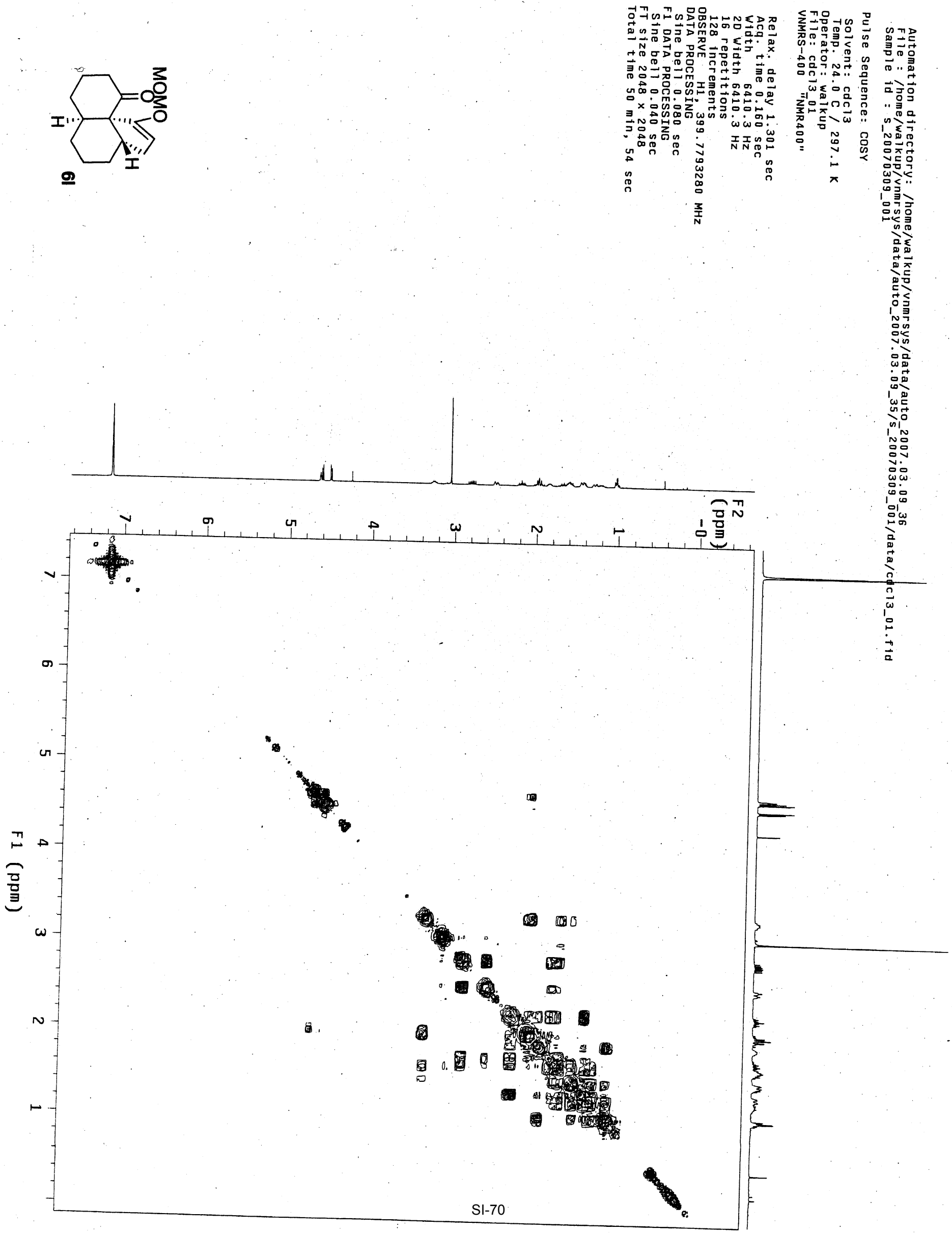

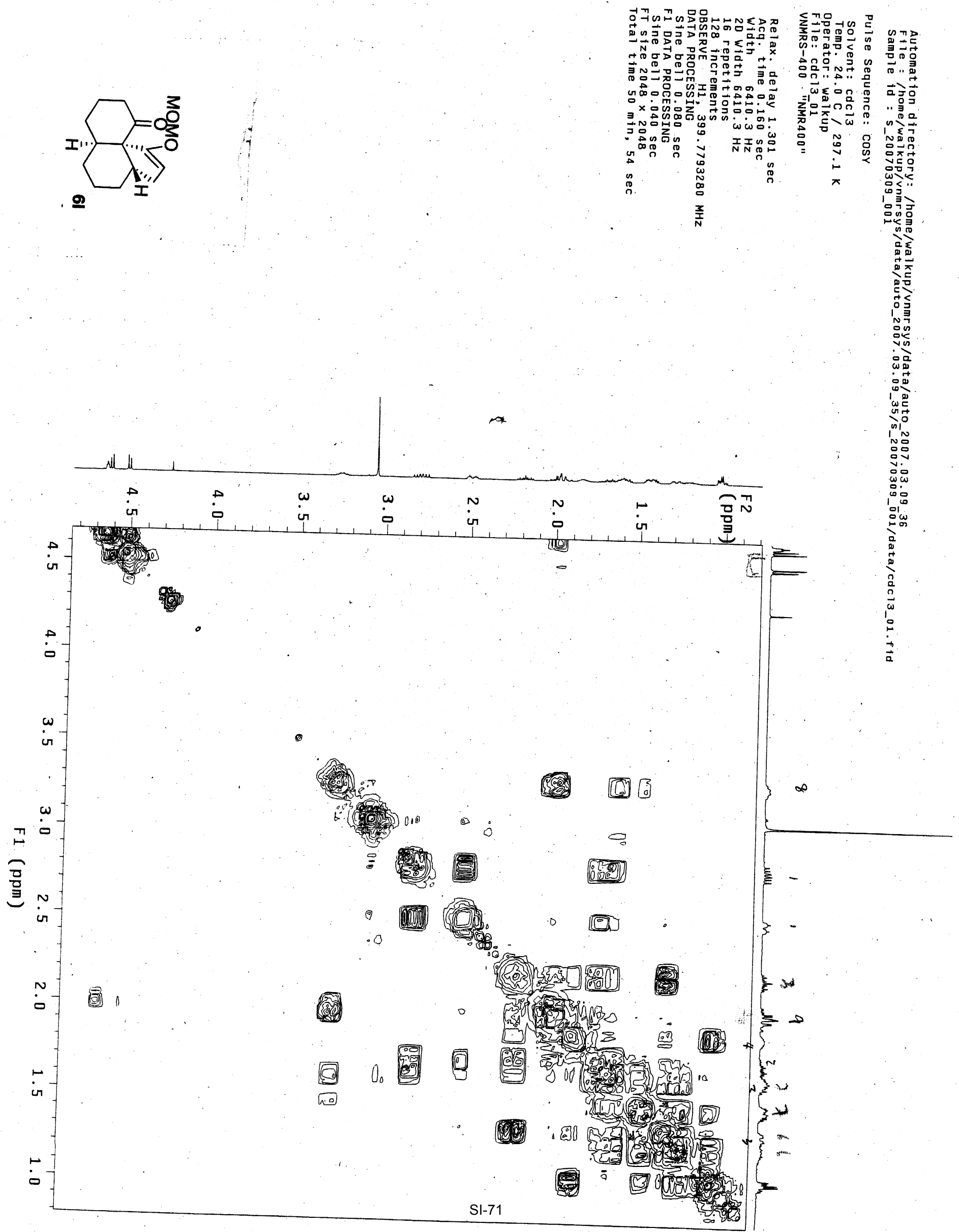


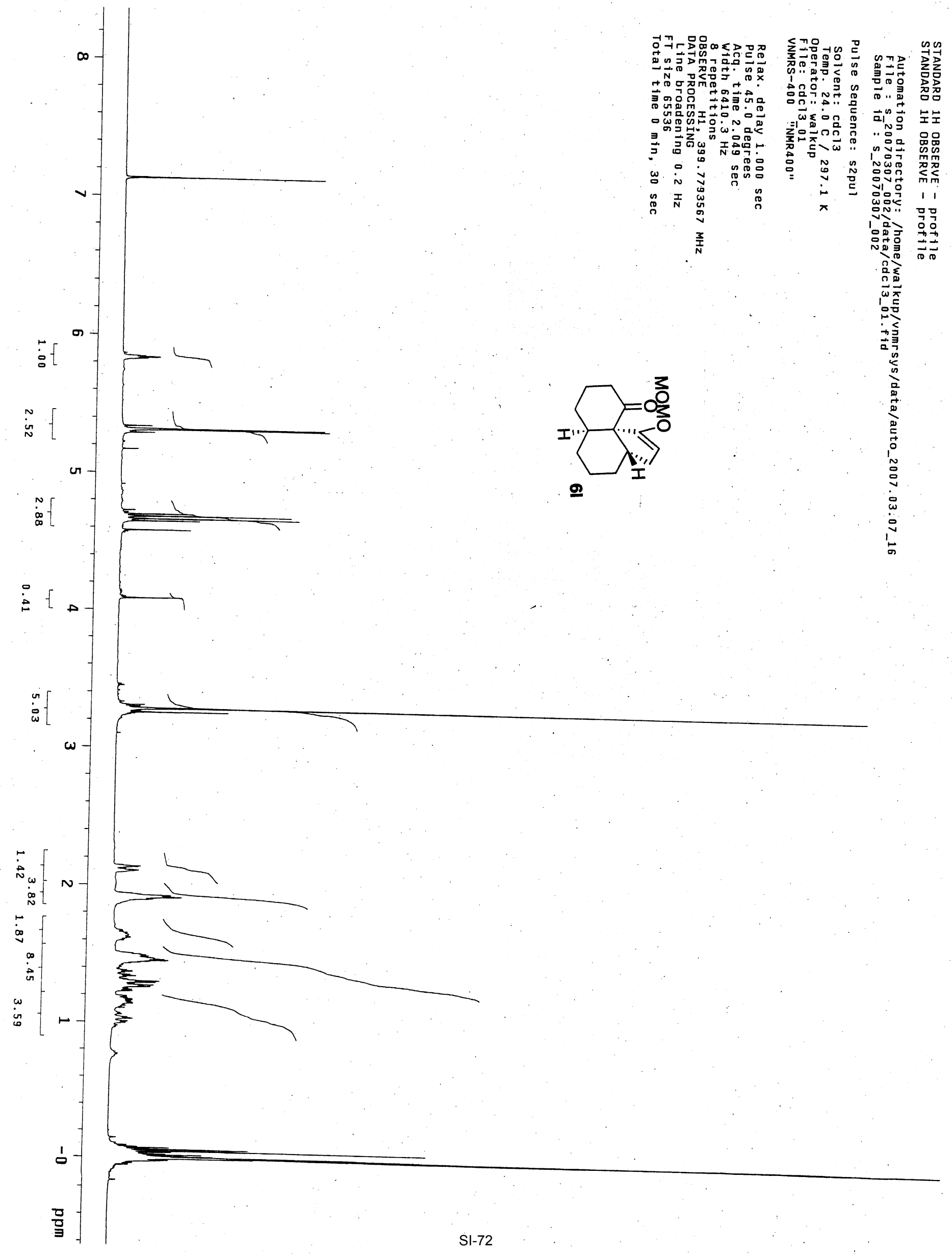




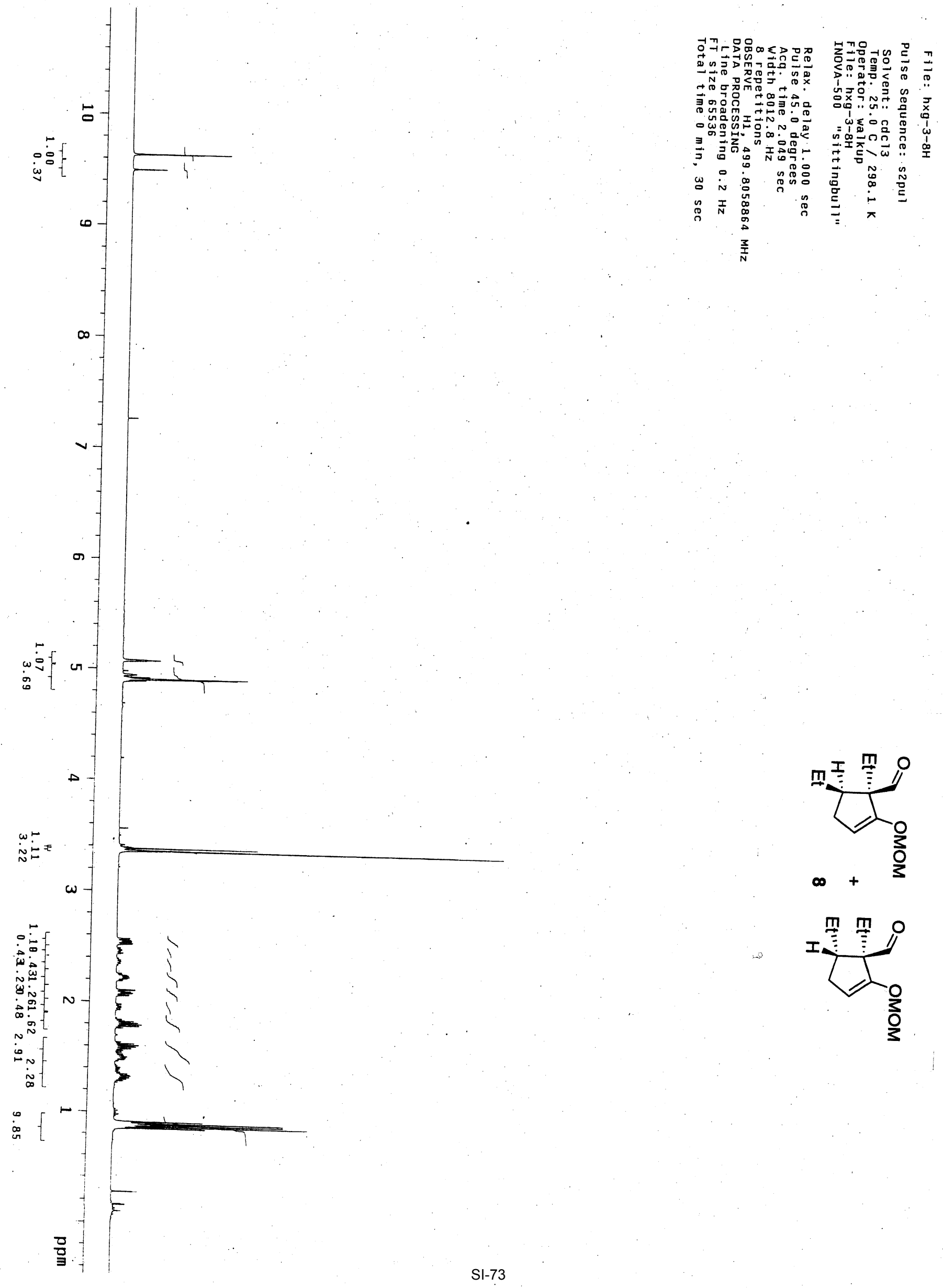




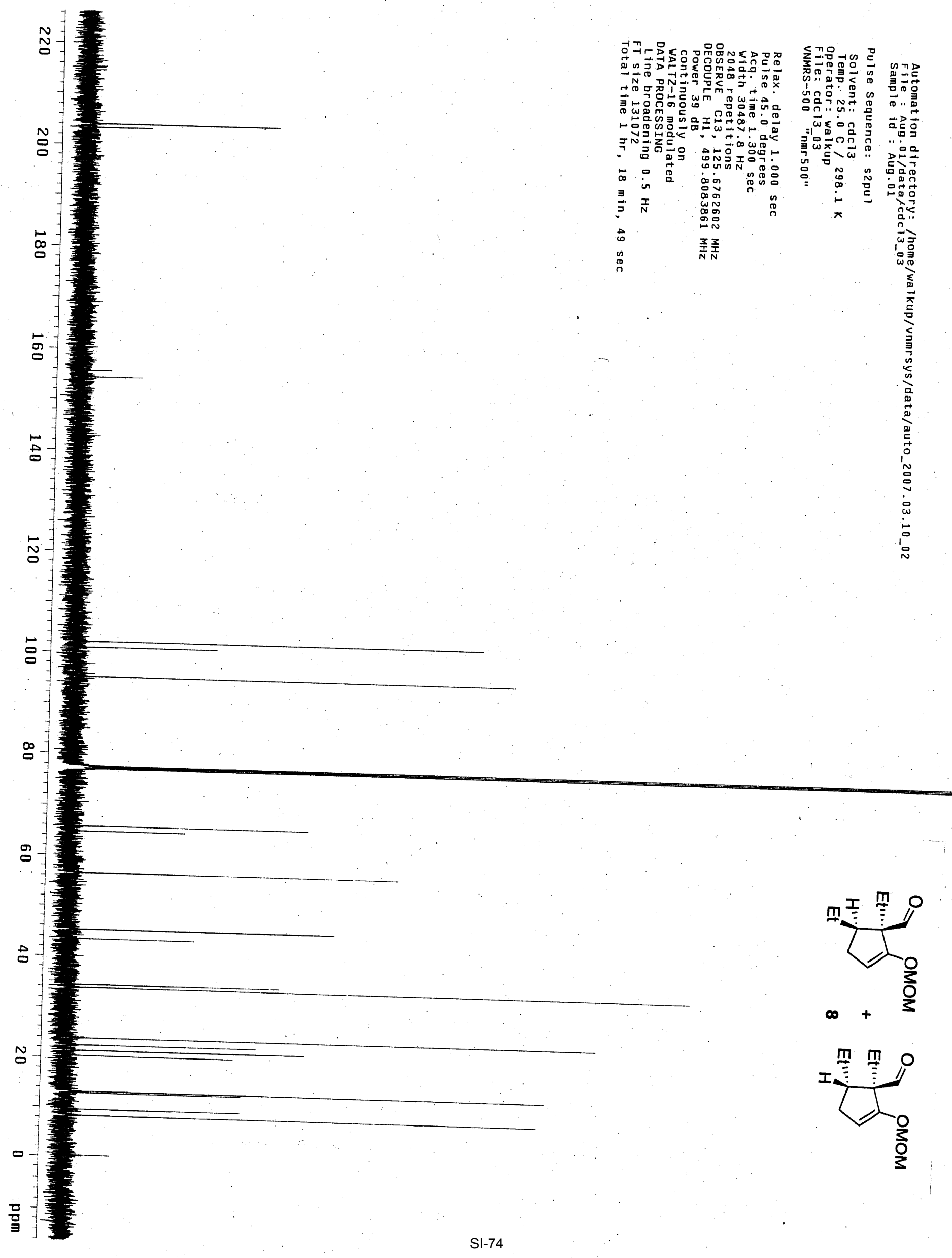




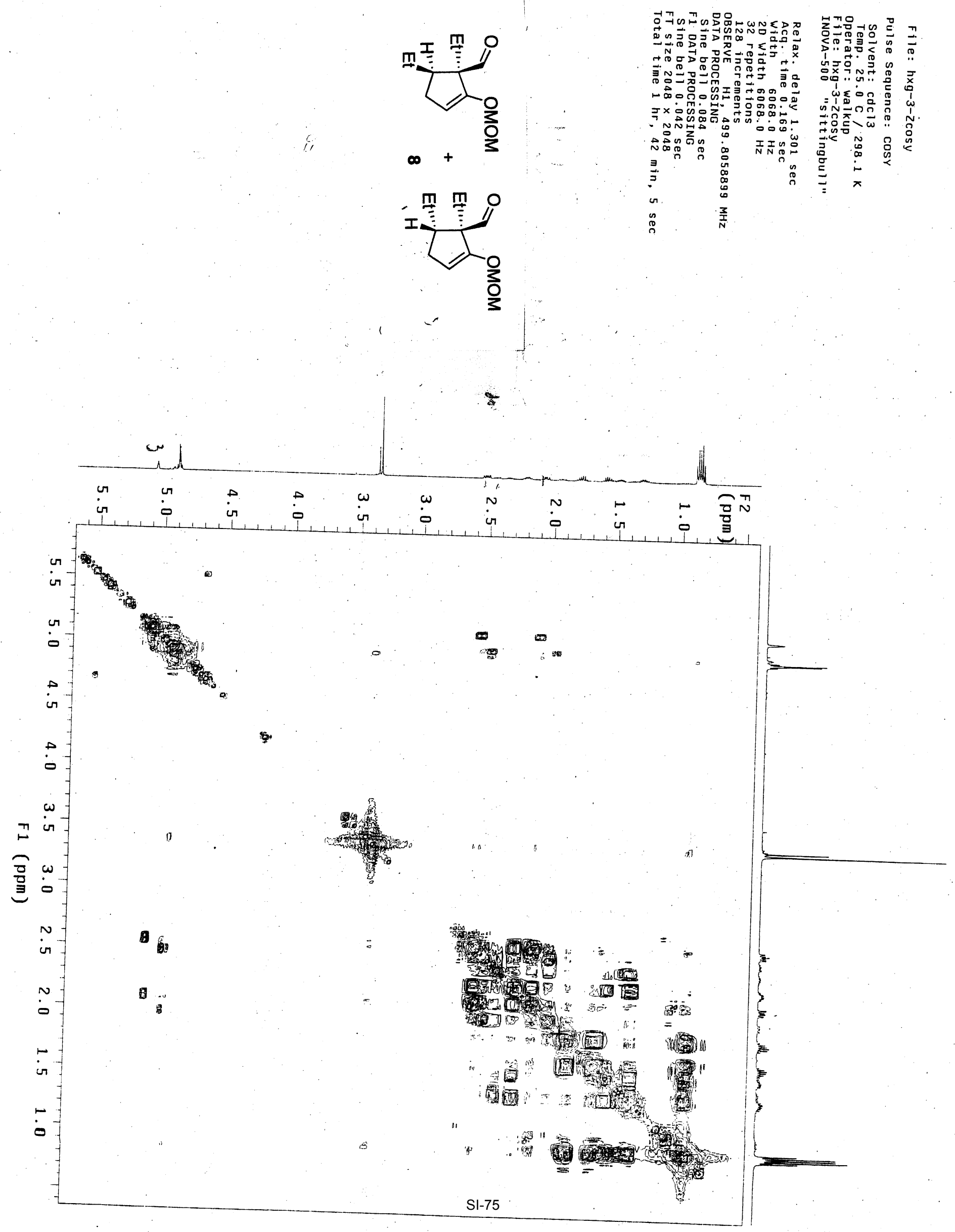




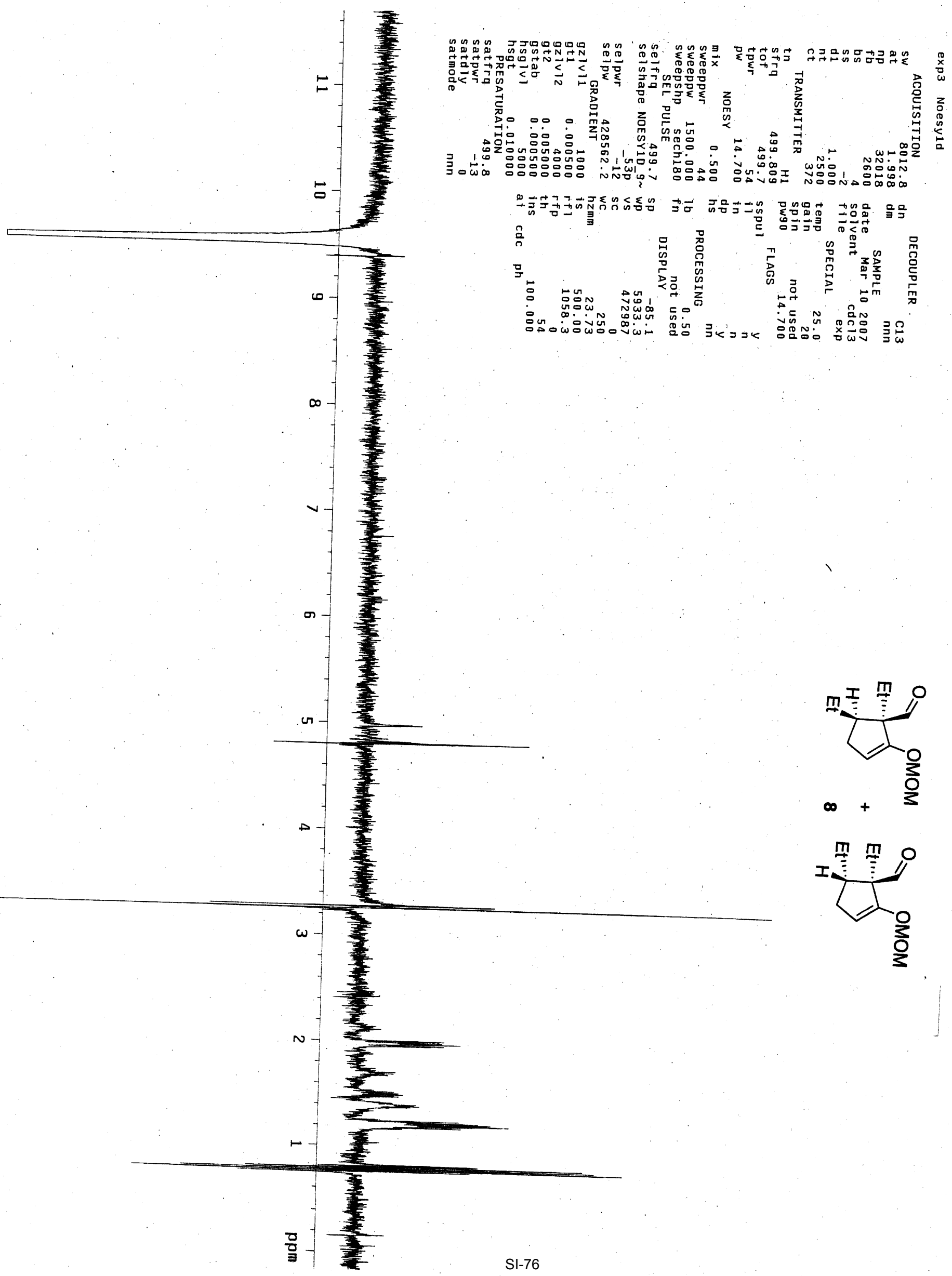




$$
1
$$




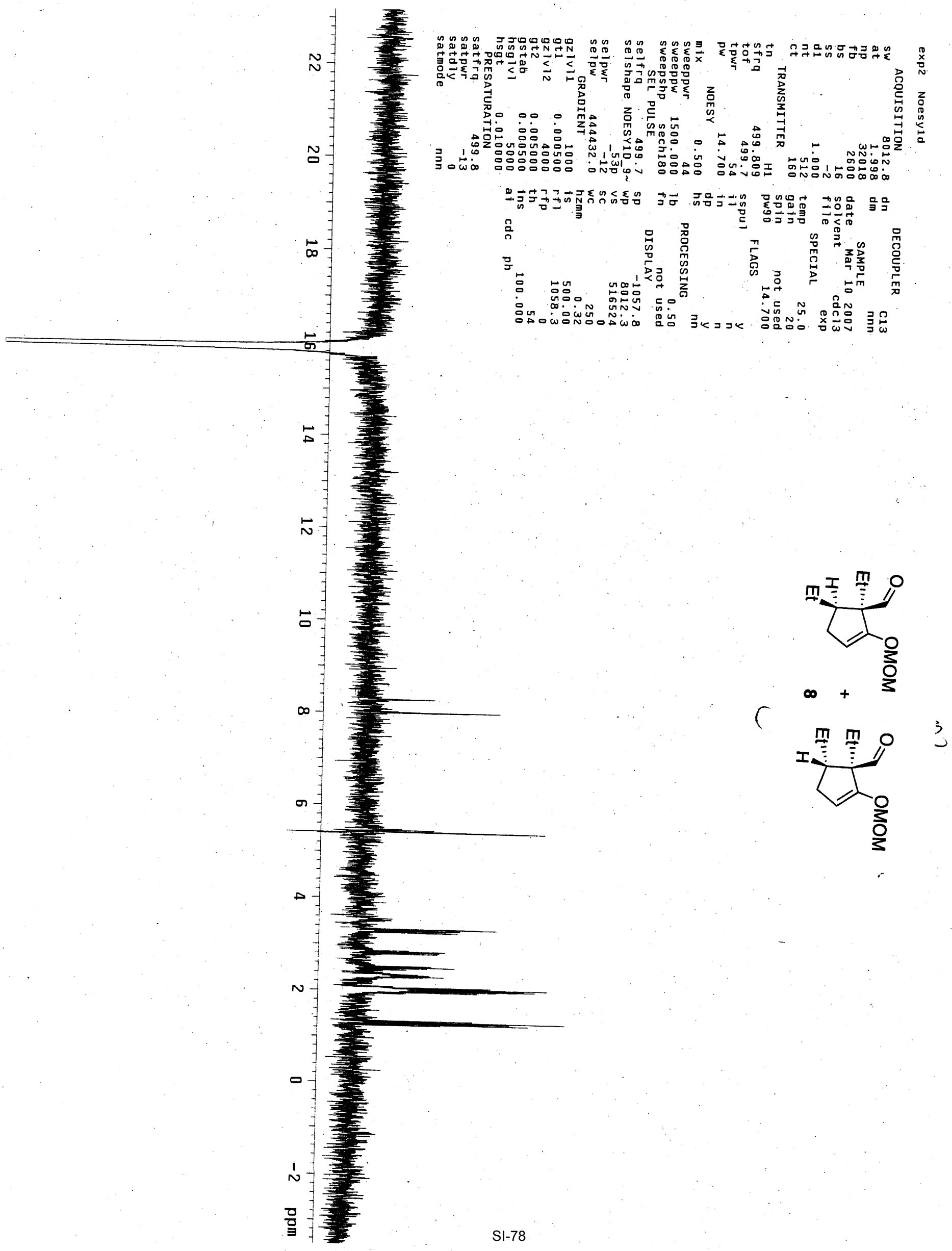

